\title{
Article
}

\section{Novel (+)-Neoisopulegol-Based O-Benzyl Derivatives as Antimicrobial Agents}

\author{
Tam Minh Le ${ }^{1,2} \mathbb{D}^{1}$, Thu Huynh ${ }^{3,4,5} \mathbb{D}^{\mathbb{D}}$, Fatima Zahra Bamou ${ }^{1} \mathbb{D}$, András Szekeres ${ }^{3,6} \mathbb{D}^{\mathbb{D}}$, Ferenc Fülöp ${ }^{1,2}$ and \\ Zsolt Szakonyi 1,6,*(D)
}

check for updates

Citation: Le, T.M.; Huynh, T.; Bamou, F.Z.; Szekeres, A.; Fülöp, F.; Szakonyi, Z. Novel (+)-NeoisopulegolBased O-Benzyl Derivatives as Antimicrobial Agents. Int. J. Mol. Sci. 2021, 22, 5626. https://doi.org/ $10.3390 /$ ijms 22115626

Academic Editor: Andrea Spallarossa

Received: 24 April 2021

Accepted: 18 May 2021

Published: 26 May 2021

Publisher's Note: MDPI stays neutral with regard to jurisdictional claims in published maps and institutional affiliations.

Copyright: (c) 2021 by the authors. Licensee MDPI, Basel, Switzerland. This article is an open access article distributed under the terms and conditions of the Creative Commons Attribution (CC BY) license (https:/ / creativecommons.org/licenses/by/ $4.0 /)$.
1 Institute of Pharmaceutical Chemistry, University of Szeged, Interdisciplinary Excellent Center, Eötvös utca 6 , H-6720 Szeged, Hungary; leminhtam1411@gmail.com (T.M.L.);

Bamou.Fatima.Zahra@stud.u-szeged.hu (F.Z.B.); fulop.ferenc@szte.hu (F.F.)

2 Stereochemistry Research Group of the Hungarian Academy of Sciences, Eötvös utca 6 H-6720 Szeged, Hungary

3 Department of Microbiology, University of Szeged, Közép fasor 52, 6726 Szeged, Hungary; huynh_thu@hcmut.edu.vn (T.H.); andras.j.szekeres@gmail.com (A.S.)

4 Department of Biotecnology, Faculty of Chemical Engineering, Ho Chi Minh University of Technology (HCMUT), 268 Ly Thuong Kiet Street, District 10, Ho Chi Minh City 72607, Vietnam

5 Vietnam National University Ho Chi Minh City, Linh Trung Ward, Thu Duc District, Ho Chi Minh City 71351, Vietnam

6 Interdisciplinary Centre of Natural Products, University of Szeged, Eötvös utca 6, H-6720 Szeged, Hungary

* Correspondence: szakonyi.zsolt@szte.hu; Tel.: +36-62-546809; Fax: +36-62-545705

\begin{abstract}
Discovery of novel antibacterial agents with new structures, which combat pathogens is an urgent task. In this study, a new library of (+)-neoisopulegol-based $O$-benzyl derivatives of aminodiols and aminotriols was designed and synthesized, and their antimicrobial activity against different bacterial and fungal strains were evaluated. The results showed that this new series of synthetic $O$-benzyl compounds exhibit potent antimicrobial activity. Di-O-benzyl derivatives showed high activity against Gram-positive bacteria and fungi, but moderate activity against Gram-negative bacteria. Therefore, these compounds may serve a good basis for antibacterial and antifungal drug discovery. Structure-activity relationships were also studied from the aspects of stereochemistry of the O-benzyl group on cyclohexane ring and the substituent effects on the ring system.
\end{abstract}

Keywords: (+)-neoisopulegol; O-Benzyl derivatives; imidazole; 1,2,4-triazole; aminodiol; aminotriol

\section{Introduction}

Heterocyclic compounds, occurring both naturally and produced synthetically, exhibit various pharmacological and biological properties and, therefore, they are interesting synthetic targets in the search of therapeutic agents [1,2]. O-Benzyl azole derivatives have played crucial roles in the history of heterocyclic chemistry and have been used extensively as important pharmacophores and synthons in the field of organic chemistry and drug design [1]. Azoles such as imidazole [3] and triazole [4] are the most extensively studied classes of antifungal agents due to their high therapeutic index, good bioavailability, and favorable safety profile [5] while the O-benzyl substituent plays an important role in the increased antimicrobial activity of these molecules [6] (Figure 1).

O-Benzyl-1,2,4-triazole derivatives were reported to exhibit various pharmacological activities such as antimicrobial [7,8], analgesic [9], anti-inflammatory [10], anticancer [8], antitubercular [11], anti-HIV [12], and antioxidant [13] properties. In addition, drugs with chemotherapeutic effect such as Anastrozole [14] and Letrozole [15] (chemotherapeutic anticancer drug), Ribavirin [16-19] (antiviral agent), Rizatriptan [20] (antimigraine agent), Alprazolam [21] (anxiolytic agent), Fluconazole [22], and Itraconazole [23] (antifungal agent) as well as Prothioconazole [21] (plant-pathogenic effect) are examples of potent molecules possessing a triazole nucleus [24,25]. 
O-Benzyl imidazole derivatives have evoked considerable attention in recent years because these are endowed with a wide range of pharmaceutical activities. These include antifungal [26], antiparasitic [27], antigiardiasis [28], antitubercular [29], antihistaminic [30], antineuropathic [31], antiobesity [32], antihypertensive [33], antioxidant [34], cardiotonic [35], antithrombotic [36], anti-convulsant [37,38], antiviral [39], and anti-hepatitis B and C virus activity [40] and they may also act as HIV-IPR [41] and IL-1 [42] inhibitors. In particular, a large number of imidazole-based compounds have been widely used drugs such as anticancer $[43,44]$ (dacarbazine, zoledronicacid, azathioprine, and tipifarnib), antifungal $[45,46]$ (clotrimazole, miconazole, ketoconazole, and oxiconazole), antibacterial [47,48] (metronidazole, ornidazole, and secnidazole), antiprotozoal [49-54] (megazol, benznidazole, and metronidazole), antihistaminic [55-57] (cimetidine, imetit, immepip, and thioperamide), antineuropathic [31,58-64] (nafimidone, fipamezole, and dexmedetomidine), and antihypertensive $[65,66]$ (losartan, eprosartan, and olmesartan) agents to treat various types of diseases with high therapeutic potency, which shows their huge development value [40].<smiles>OC(Cn1cncn1)(Cn1cncn1)c1ccc(F)cc1F</smiles>

Fluconazole<smiles>Clc1ccccc1C(c1ccccc1)(c1ccccc1)n1ccnc1</smiles>

Clotrimazole<smiles>Cc1ncc([N+](=O)[O-])n1CCO</smiles>

Metronidazole<smiles>CC(C)n1ncn(-c2ccc(N3CCN(c4ccc(OC[C@@H]5COC(Cn6cncn6)(c6ccc(Cl)cc6Cl)O5)cc4)CC3)cc2)c1=O</smiles>

Itraconazole<smiles>Clc1ccc(COC(Cn2ccnc2)c2ccc(Cl)cc2Cl)c(Cl)c1</smiles>

Miconazole<smiles>Cc1ncc([N+](=O)[O-])n1CC(O)CCl</smiles>

Ornidazole<smiles>CC(=O)N1CCN(c2ccc(OC[C@H]3COC(c4ccc(Cl)cc4Cl)(n4ccnc4)O3)cc2)CC1</smiles>

Ketoconazole<smiles>Cc1ncc([N+](=O)[O-])n1CC(C)O</smiles>

Secnidazole

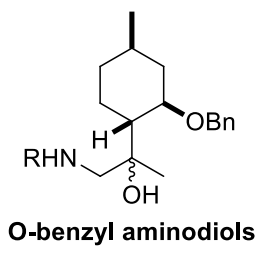

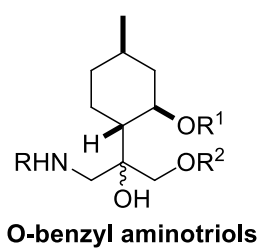

$\mathrm{R}^{1}=\mathrm{R}^{2}=\mathrm{H}$ or $\mathrm{Bn}$

$\mathrm{R}=\mathrm{CH}_{2} \mathrm{Ph}, \mathrm{CH}\left(\mathrm{CH}_{3}\right) \mathrm{Ph}$, $\mathrm{CH}\left(\mathrm{CH}_{3}\right)_{2}$

Figure 1. Azoles as potent antimicrobial agents.

The increasing number of multidrug-resistant pathogen infections has led to the discovery of new antimicrobial drugs with activity against resistant clinical isolates [67]. In our long-term program toward the synthesis of new antimicrobial agents, we demonstrated that (-)-isopulegol-based O-benzyl aminotriol and aminodiol derivatives exert marked antimicrobial effectiveness [68]. Therefore, the present study reports the synthesis of a series of novel (+)-neoisopulegol-based $O$-benzyl derivatives of aminodiols and aminotriols with nitrogen atoms usually incorporated in an imidazole or triazole ring system possessing activity against various bacteria and yeast strains. According to their antimicrobial activities, structure-activity relationships have also been discussed. 


\section{Results}

\subsection{Synthesis of (+)-Neoisopulegol-Based O-Benzyl Derivatives}

(+)-Neoisopulegol 2 was prepared from commercially available (-)-isopulegol 1 by oxidizing its hydroxyl function followed by the stereoselective reduction of the resulting carbonyl group applying literature methods [69-72]. In order to produce O-benzyl derivatives, benzyl-protected neoisopulegol 3 was prepared by reacting of 2 with $\mathrm{BnBr}$ in the presence of a catalytic amount of KI [73,74]. Without the addition of KI, the reaction proceeded very slowly whereas with the addition of 1 equiv. of KI, the reaction proceeded rapidly due to the formation of more reactive $\mathrm{BnI}$ from $\mathrm{BnBr}$ [75]. Epoxidation of 3 with m-CPBA buffered with $\mathrm{Na}_{2} \mathrm{HPO}_{4}$ provided a 1:2 mixture of epoxides $4 \mathbf{a}$ and $4 \mathbf{b}$ in good yield good yields [76]. The two epoxides were separated by column chromatography to give less polar isomer $\mathbf{4 a}$ and more polar isomer $\mathbf{4} \mathbf{b}$. Aminolysis of epoxide $\mathbf{4 a}$ with different amines in the presence of $\mathrm{LiClO}_{4}$ delivered $O$-benzyl derivatives 5a-6a [77,78]. The role of $\mathrm{LiClO}_{4}$ shows enhanced reactivity for the ring opening of epoxides through the coordination of $\mathrm{Li}^{+}$with epoxide oxygen, rendering the epoxide more susceptible to nucleophilic attack by amines, therefore reducing the reaction times dramatically and improved the yields $[79,80]$. Likewise, no products were observed during ring-opening of the oxirane $3 \mathbf{a}$ with azoles and $\mathrm{LiClO}_{4}$. This is probably the difference in reactivity between amines and azoles. Fortunately, it was achieved by reacting $4 \mathbf{a}$ with azoles promoted by $\mathrm{K}_{2} \mathrm{CO}_{3}$ [81]. A possible reaction pathway through potassium carbonate-mediated ring-opening reaction of epoxide $4 \mathbf{a}$ and subsequent nucleophilic addition afforded $O$-benzyl derivatives 7a-8a [82] Debenzylation of $\mathbf{5 a}$ by hydrogenolysis over $\mathrm{Pd} / \mathrm{C}$ in $\mathrm{MeOH}$ resulted in primary aminodiol 9a in excellent yield. Since neither aminolysis of the served oxirane $4 a$ in alkaline condition nor the hydrogenolysis of $\mathrm{N}$-benzyl analogue $5 \mathbf{a}$ had an effect on the absolute configuration, the relative configuration of the chiral centers of $\mathbf{5 a - 9 a}$ is known to be the same as that of epoxide $\mathbf{4 a}[83,84]$. The other epoxide (4b) underwent similar reactions to afford $\mathbf{5 b} \mathbf{b} \mathbf{9} \mathbf{b}$ in valuable yields (Scheme 1).

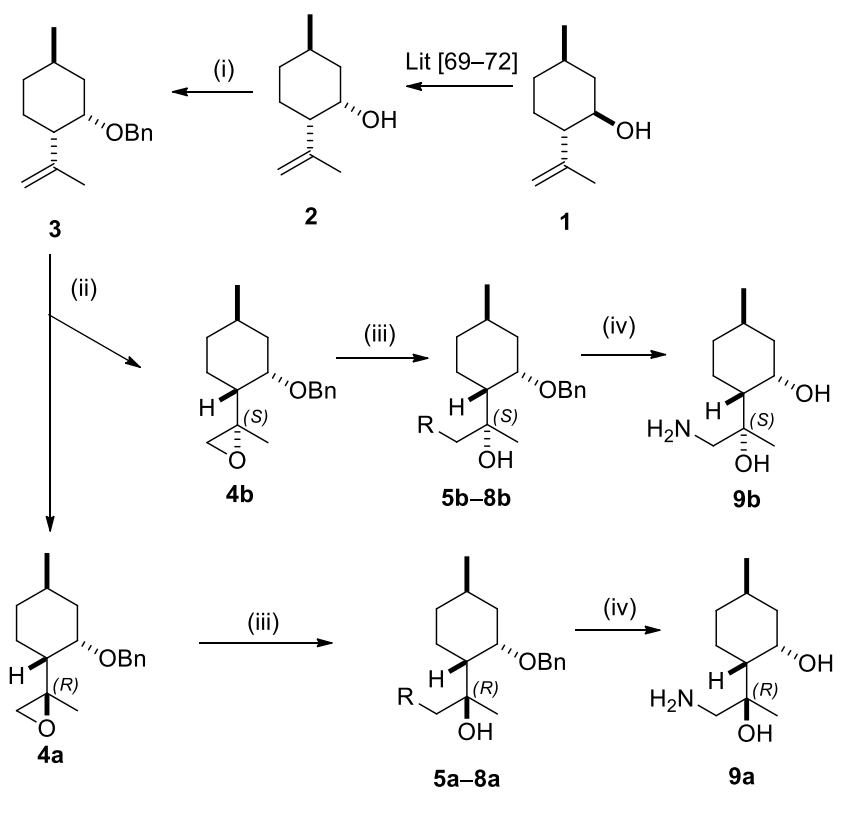

5a-b: $\mathrm{R}=\mathrm{NHCH}_{2} \mathrm{Ph} ; \mathbf{6 a}-\mathbf{b}: \mathrm{R}=\mathrm{N}\left(\mathrm{CH}_{2} \mathrm{Ph}\right)_{2} ; \mathbf{7 a}-\mathbf{b}: \mathbf{R}=$ imidazole; $\mathbf{8} \mathbf{a}-\mathbf{b}:$ 1,2,4-triazole

Scheme 1. Synthesis of (+)-neoisopulegol-based $O$-benzyl aminodiols. Reaction conditions: (i) $\mathrm{NaH}$ (1 equ.), $\mathrm{BnBr}$ (1.5 equ.), $\mathrm{KI}$ (1 equ.), dry THF, $60{ }^{\circ} \mathrm{C}, 12 \mathrm{~h}, 63 \%$; (ii) $m$-CPBA (2 equ.), $\mathrm{Na}_{2} \mathrm{HPO}_{4}$. $12 \mathrm{H}_{2} \mathrm{O}$ (3 equ.), $\mathrm{CH}_{2} \mathrm{Cl}_{2}, 25^{\circ} \mathrm{C}, 2 \mathrm{~h}, 23 \%$ (4a), $47 \%$ (4b); (iii) $\mathrm{R}^{1} \mathrm{R}^{2} \mathrm{NH}$ (2 equ.), $\mathrm{LiClO}_{4}$ (1 equ.), $\mathrm{MeCN}$, $70-80{ }^{\circ} \mathrm{C}, 20 \mathrm{~h}, 25-78 \%$ (for $\mathbf{5 a}-\mathbf{b}$ and $\mathbf{6 a}-\mathbf{b}$ ) or imidazole/1,2,4-triazol (3 equ.), $\mathrm{K}_{2} \mathrm{CO}_{3}$ (5 equ.), dry DMF, 70-80 ${ }^{\circ} \mathrm{C}, 24 \mathrm{~h}, 42-67 \%$ (for $7 \mathbf{a}-\mathbf{b}$ and $8 \mathbf{a}-\mathbf{b}$ ); (iv) $5 \% \mathrm{Pd} / \mathrm{C}, \mathrm{H}_{2}\left(1 \mathrm{~atm}\right.$ ), $\mathrm{MeOH}, 25^{\circ} \mathrm{C}, 24 \mathrm{~h}, 91 \%$ (from $5 \mathbf{a}$ or $\mathbf{5 b}$ ). 
To prepare a highly diverse library of $O$-benzyl aminotriols, 3 was oxidized to 10 using $\mathrm{SeO}_{2} / t$-BuOOH (TBHP) as oxidant [85]. The epoxidation of $\mathbf{1 0}$ with $m$-CPBA delivered a 4:1 mixture of epoxides 11a and $\mathbf{1 1} \mathbf{b}$. The separation of $\mathbf{1 1 a}$ and $\mathbf{1 1} \mathbf{b}$ was not satisfactory on a gram scale; therefore, the mixture was treated with different nucleophiles resulting in a library of $O$-benzyl derivatives 12-15. In our delight, amine-substituted O-benzyl derivatives could easily be separated while in the case of azoles, only the major products were isolated. The debenzylation of 12a by hydrogenolysis over $\mathrm{Pd} / \mathrm{C}$ gave primary aminotriol 16a with good yield (Scheme 2).

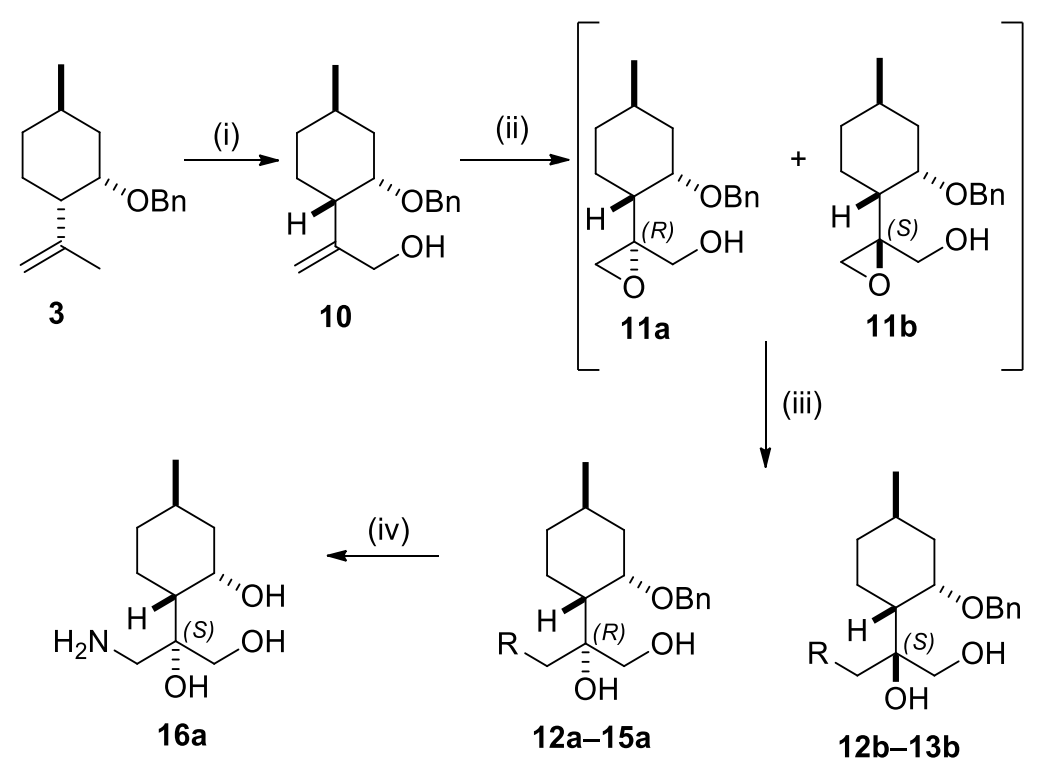

12a-b: $\mathrm{R}=\mathrm{NHCH}_{2} \mathrm{Ph} ; 13 \mathbf{a}-\mathbf{b}: \mathrm{R}=\mathrm{N}\left(\mathrm{CH}_{2} \mathrm{Ph}\right)_{2} ; 14 \mathbf{a}: \mathrm{R}=$ imidazole; 15a: 1,2,4-triazole

Scheme 2. Synthesis of (+)-neoisopulegol-based O-benzyl aminotriols. Reaction conditions: (i) $\mathrm{SeO}_{2}$ (0.24 equ.), $70 \% \mathrm{t}$-BuOOH ( 4 equ.), $\mathrm{CHCl}_{3}, 25^{\circ} \mathrm{C}, 20 \mathrm{~h}$, then $\mathrm{NaBH}_{4}$ ( 3 equ.), dry $\mathrm{MeOH}, 0{ }^{\circ} \mathrm{C}, 2 \mathrm{~h}, 27 \%$; (ii) $m$-CPBA (2 equ.), $\mathrm{Na}_{2} \mathrm{HPO}_{4} \cdot 12 \mathrm{H}_{2} \mathrm{O}$ (3 equ.), $\mathrm{CH}_{2} \mathrm{Cl}_{2}, 25^{\circ} \mathrm{C}, 2 \mathrm{~h}, 60 \%$ (11a $+\mathbf{1 1 b}$ ); (iii) $\mathrm{R}^{1} \mathrm{R}^{2} \mathrm{NH}(2$ equ.), $\mathrm{LiClO}_{4}$ (1 equ.), $\mathrm{MeCN}, 70-80{ }^{\circ} \mathrm{C}, 8 \mathrm{~h}, 7-54 \%$ (for 12a-b and 13a-b) or imidazole/1,2,4-triazol (3 equ.), $\mathrm{K}_{2} \mathrm{CO}_{3}$ (5 equ.), dry DMF, $70-80{ }^{\circ} \mathrm{C}, 12 \mathrm{~h}, 14 \mathrm{a}: 58 \%, 15 \mathrm{a}: 46 \%$; (iv) $5 \% \mathrm{Pd} / \mathrm{C}, \mathrm{H}_{2}(1 \mathrm{~atm})$, $\mathrm{MeOH}, 25^{\circ} \mathrm{C}, 24 \mathrm{~h}, 78 \%$ (from 12a).

During our attempt to improve the separation of epoxides 11a-b, we realized that $O$-benzylation of $\mathbf{1 0}$ could serve this purpose. The synthesis of 18a starting from 10 with $\mathrm{NaH} / \mathrm{BnBr} / \mathrm{KI}$ system, however, provided low-yield transformation (20\%). Fortunately, it was achieved starting from 17, made by the oxidation of 2 [69-72]. Diol 17 was reacted with benzyl bromide under reflux condition in dry THF to give $\mathbf{1 8 a}$, whereas $\mathbf{1 8 b}$ was prepared at room temperature. Epoxidation of 18a with $m$-CPBA produced a 1:1 mixture of epoxides $19 a$ and $19 b$. After purification, ring opening of oxiranes 19a-b was accomplished with different nucleophiles resulting in a library of di-O-benzyl derivatives 20a-24a and 20b$24 \mathrm{~b}$, respectively. The debenzylation of $20 \mathrm{a}$ and $\mathbf{2 0 b}$ by hydrogenolysis over $\mathrm{Pd} / \mathrm{C}$ gave, respectively, primary aminotriols $\mathbf{1 6 a}$ and $\mathbf{1 6 b}$ in exceptionally high yields (Scheme 3). 

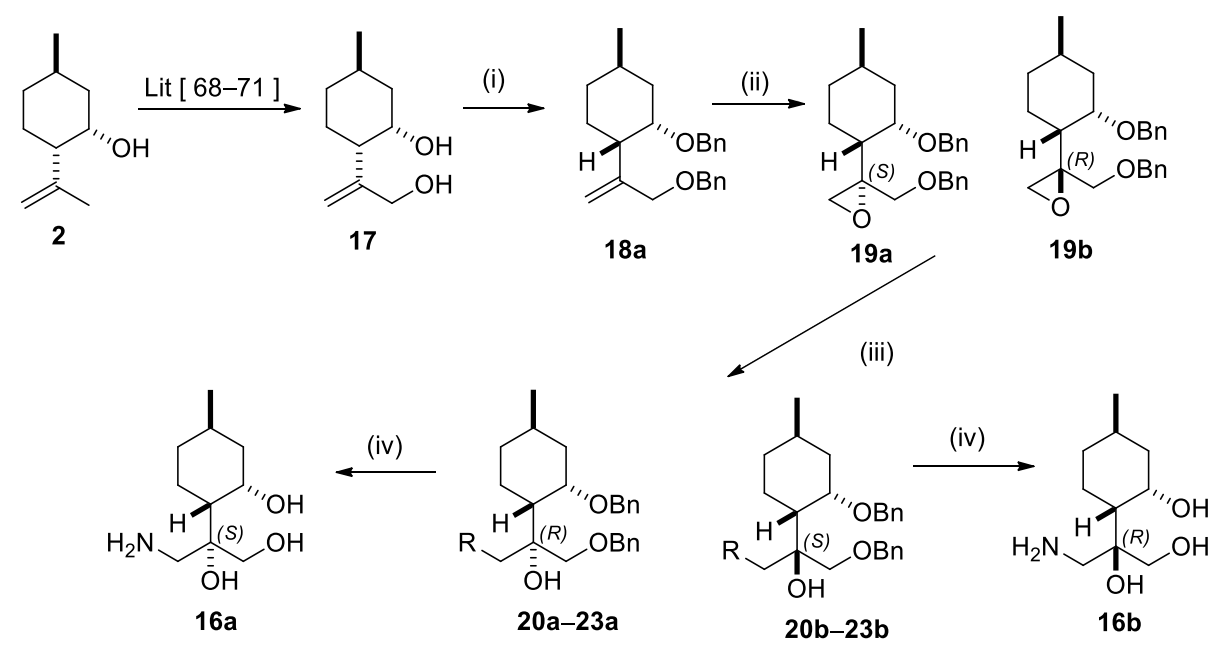

20a-b: $\mathrm{R}=\mathrm{NHCH}_{2} \mathrm{Ph} ; \mathbf{2 1} \mathbf{a}-\mathbf{b}: \mathrm{R}=\mathrm{N}\left(\mathrm{CH}_{2} \mathrm{Ph}\right)_{2} ; \mathbf{2 2} \mathbf{a}-\mathbf{b}: \mathbf{R}=$ imidazole; $23 \mathbf{a}-\mathbf{b}:$ 1,2,4-triazole

Scheme 3. Synthesis of (+)-neoisopulegol-based di-O-benzyl aminotriols. Reaction conditions: (i) $\mathrm{NaH}$ (1 equ.), $\mathrm{BnBr}$ (1.5 equ.), $\mathrm{KI}$ (1 equ.), dry THF, $60{ }^{\circ} \mathrm{C}, 24 \mathrm{~h}, 56 \%$; (ii) $m$-CPBA (2 equ.), $\mathrm{Na}_{2} \mathrm{HPO}_{4}$. $12 \mathrm{H}_{2} \mathrm{O}$ (3 equ.), $\mathrm{CH}_{2} \mathrm{Cl}_{2}, 25{ }^{\circ} \mathrm{C}, 2 \mathrm{~h}, 36 \%$ (19a), 36\% (19b); (iii) $\mathrm{R}^{1} \mathrm{R}^{2} \mathrm{NH}$ (2 equ.), $\mathrm{LiClO}_{4}$ (1 equ.), $\mathrm{MeCN}, 70-80{ }^{\circ} \mathrm{C}, 6 \mathrm{~h}, 53-84 \%$ (for 20a-b and 21a-b) or imidazole/1,2,4-triazol (3 equ.), $\mathrm{K}_{2} \mathrm{CO}_{3}(5$ equ.), dry DMF, $70-80{ }^{\circ} \mathrm{C}, 48 \mathrm{~h}, 42-67 \%$ (for 22a-b and 23a-b); (iv) 5\% Pd/C, $\mathrm{H}_{2}$ (1 atm), $\mathrm{MeOH}$, $25^{\circ} \mathrm{C}, 24 \mathrm{~h}, 94 \%$ (from $20 \mathrm{a}$ or $20 \mathrm{~b}$ ).

The epoxidation of $\mathbf{1 8 b}$ with $m$-CPBA gave a 3:1 mixture of epoxides $\mathbf{2 4 a}$ and $\mathbf{2 4 b}$. After separation by column chromatography, they were subjected to aminolysis with different nucleophiles to form a library of $O$-benzyl derivatives $\mathbf{2 5 a} \mathbf{a} \mathbf{2 8} \mathbf{a}$ and $\mathbf{2 5} \mathbf{b}-\mathbf{2 8} \mathbf{b}$, respectively. Primary aminotriols $\mathbf{1 6 a}$ and $\mathbf{1 6 b}$ were prepared via the usual way by hydrogenolysis of aminodiols $\mathbf{2 5 a}$ and $\mathbf{2 5 b}$ over Pd/C (Scheme 4).

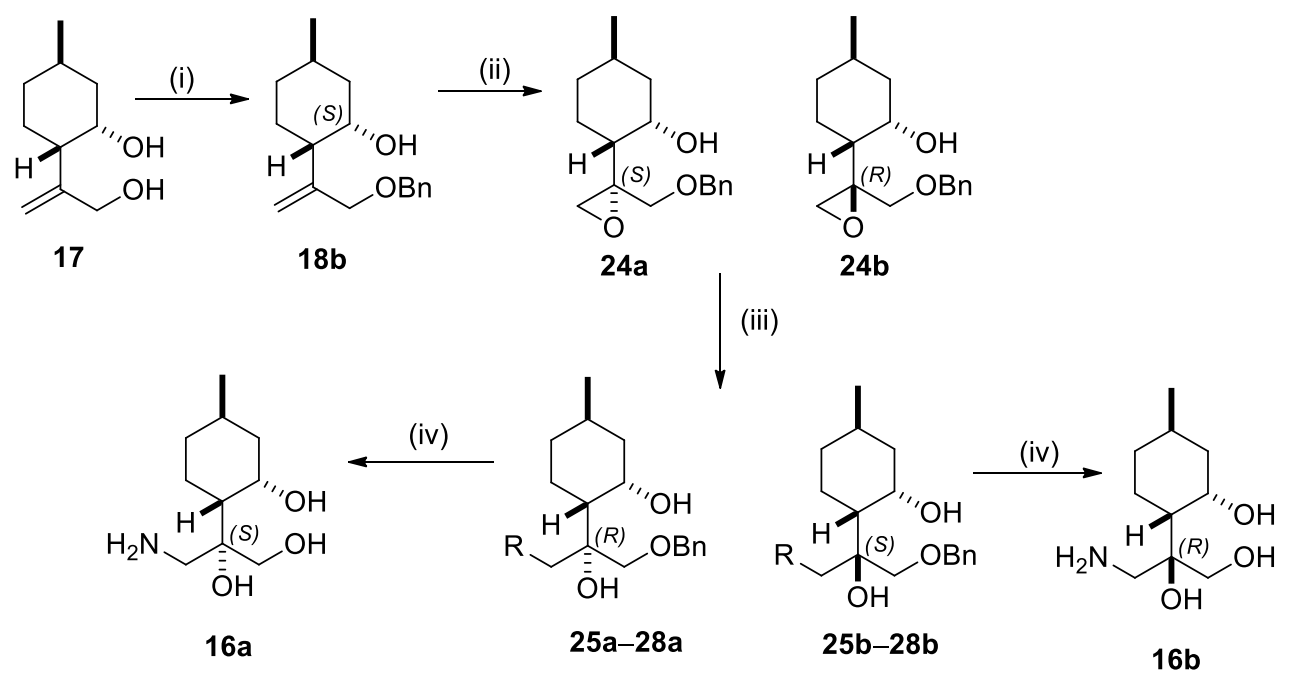

25a-b: $\mathrm{R}=\mathrm{NHCH}_{2} \mathrm{Ph} ; \mathbf{2 6 a}-\mathbf{b}: \mathrm{R}=\mathrm{N}\left(\mathrm{CH}_{2} \mathrm{Ph}\right)_{2} ; \mathbf{2 7 a} \mathbf{a}-\mathbf{b}: \mathrm{R}=$ imidazole; 28a-b: 1,2,4-triazole

Scheme 4. Synthesis of (+)-neoisopulegol-based O-benzyl aminotriols. Reaction conditions: (i) $\mathrm{NaH}$ (1 equ.), $\mathrm{BnBr}$ (1.5 equ.), KI (1 equ.), dry THF, $24{ }^{\circ} \mathrm{C}, 24 \mathrm{~h}, 59 \%$; (ii) $m$-CPBA (2 equ.), $\mathrm{Na}_{2} \mathrm{HPO}_{4}$. $12 \mathrm{H}_{2} \mathrm{O}$ (3 equ.), $\mathrm{CH}_{2} \mathrm{Cl}_{2}, 25{ }^{\circ} \mathrm{C}, 2 \mathrm{~h}, 42 \%$ (25a), 15\% (25b); (iii) $\mathrm{R}^{1} \mathrm{R}^{2} \mathrm{NH}$ (2 equ.), $\mathrm{LiClO}_{4}$ (1 equ.), $\mathrm{MeCN}, 70-80{ }^{\circ} \mathrm{C}, 8 \mathrm{~h}, 71-88 \%$ (for $\mathbf{2 5 a}-\mathbf{b}$ and $\mathbf{2 6 a}-\mathbf{b}$ ) or imidazole/1,2,4-triazol (3 equ.), $\mathrm{K}_{2} \mathrm{CO}_{3}$ (5 equ.), dry DMF, $70-80{ }^{\circ} \mathrm{C}, 12 \mathrm{~h}, 67-83 \%$ (for $27 \mathbf{a}-\mathbf{b}$ and $28 \mathbf{a}-\mathbf{b}$ ); (iv) $5 \% \mathrm{Pd} / \mathrm{C}, \mathrm{H}_{2}$ (1 atm), $\mathrm{MeOH}$, $25^{\circ} \mathrm{C}, 24 \mathrm{~h}, 91 \%$ (from $25 \mathbf{a}$ or $25 \mathbf{b}$ ). 


\subsection{Synthesis of (-)-Isopulegol-Based O-Benzyl Derivatives}

Our previous work demonstrated that the O-benzyloxy group on the cyclohexyl ring is much more effective to induce antimicrobial activity. Therefore, to explore the role of the configuration of the $O$-benzyloxy group, some (-)-isopulegol-based O-benzyl derivatives were also prepared under optimized condition and using literature information [68] (Scheme 5).
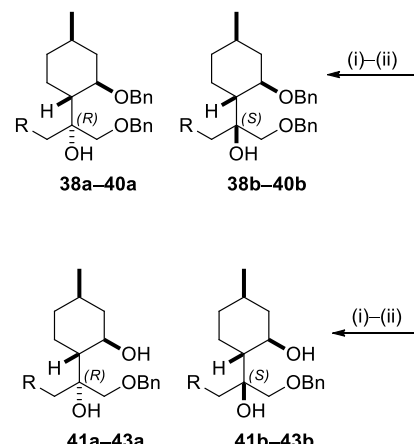

$41 a-43 a$

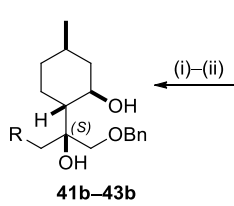

$41 b-43 b$

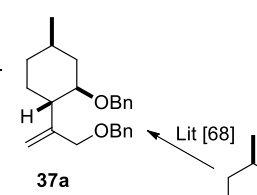

$37 \mathrm{a}$

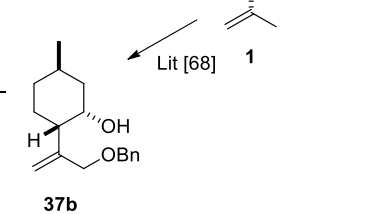
Lit [68],<smiles>C=C(C)C1CCC(C)CC1Br</smiles><smiles>[Mg][TeH2]</smiles>

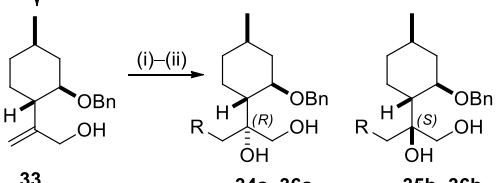

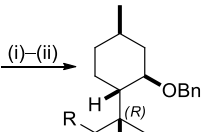<smiles>C1CCC1</smiles>

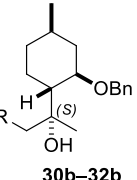

30b, 34a, 38, 41: $\mathrm{R}=\mathrm{N}\left(\mathrm{CH}_{2} \mathrm{Ph}\right)_{2} ; 31,35,39,42: \mathrm{R}=$ imidazole; 33, 35, 40, 43: 1,2,4-triazole

Scheme 5. Synthesis of (-)-isopulegol-based O-benzyl derivatives. Reaction conditions: (i) epoxidation according to our previous work [68], (ii) $\mathrm{R}^{1} \mathrm{R}^{2} \mathrm{NH}$ (2 equ.), $\mathrm{LiClO}_{4}$ (1 equ.), $\mathrm{MeCN}, 70-80{ }^{\circ} \mathrm{C}$, 6-20 h, 47\% (30b), 76\% (34a), 67-80\% (38a-b), 76-88\% (41a-b) or imidazole/1,2,4-triazol (3 equ.), $\mathrm{K}_{2} \mathrm{CO}_{3}$ (5 equ.), dry DMF, $70-80{ }^{\circ} \mathrm{C}, 12-96 \mathrm{~h}, 38-83 \%$ (for $31 \mathbf{a}-\mathbf{b}$ and $32 \mathbf{a}-\mathbf{b}$ ), 50-67\% (for $35 \mathbf{a}-\mathbf{b}$ and $36 \mathbf{a}-\mathbf{b}$ ), $67-83 \%$ (for $39 \mathbf{a}-\mathbf{b}$ and $40 \mathbf{a}-\mathbf{b}$ ), $58-67 \%$ (for $41 \mathbf{a}-\mathbf{b}$ and $42 \mathbf{a}-\mathbf{b}$ ).

\subsection{Determine Relative Configuration of (+)-Neoisopulegol-Based O-Benzyl Derivatives}

Epoxidation of 2 with $t-\mathrm{BuOOH}$ in the presence of vanadyl acetylacetonate $\left(\mathrm{VO}(\mathrm{acac})_{2}\right)$ as catalyst furnished epoxide $\mathbf{4 4}$ in a stereoselective reaction [72]. Debenzylation of $\mathbf{4 b}$ provided 44 in a moderate yield whereas exposure of 44 to $\mathrm{NaOH}$ furnished 45 with the retention of stereochemistry [86]. The absolute configuration of O-benzyl derivatives 19a and 25a was determined by debenzylation together with reduction via hydrogenolysis over Pd/C $[87,88]$ to provide triol 45 with stereochemical retention [68]. The stereochemical structure of epoxide $\mathbf{4 4}$ is well-known in the literature [72]; therefore, the absolute configuration of $O$-benzyl derivatives could also be determined (Scheme 6).

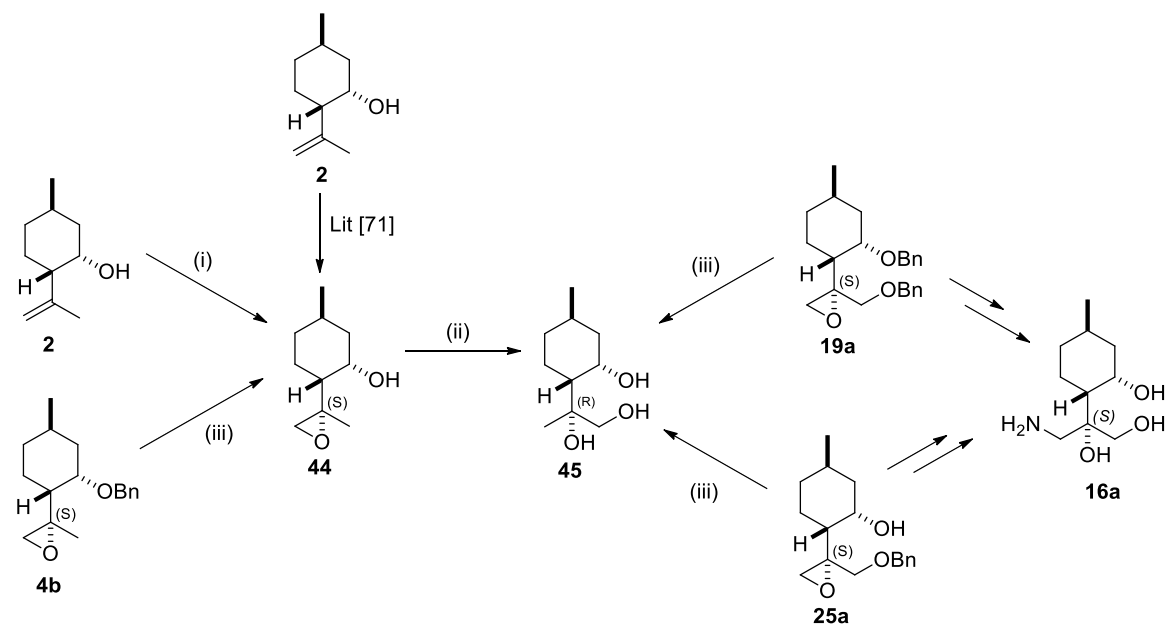

Scheme 6. Determination of the structure of (+)-neoisopulegol-based- $O$-benzyl derivatives. Reaction conditions: (i) $\mathrm{VO}(\mathrm{acac})_{2}, 70 \% \mathrm{t}$ - $\mathrm{BuOOH}$ (2 equ.), dry toluene, $25^{\circ} \mathrm{C}, 12 \mathrm{~h}, 76 \%$; (ii) $3 \mathrm{M} \mathrm{NaOH}, \mathrm{DMSO}$, $25^{\circ} \mathrm{C}, 2 \mathrm{~h}, 76 \%$; (iii) $5 \% \mathrm{Pd} / \mathrm{C}, \mathrm{H}_{2}$ (1 atm), $n$-hexane:EtOAc $=9: 1,25^{\circ} \mathrm{C}, 6-24 \mathrm{~h}, 61 \%$ (4b), 78\% (19a), $73 \%$ (25a). 


\subsection{Antimicrobial Effects}

Since several $O$-benzyl derivatives exerted antimicrobial activities on various microorganisms [68], antimicrobial activities of the prepared $O$-benzyl analogues were also explored against two yeasts as well as two Gram-positive and two Gram-negative bacteria (Table 1, only the best results are shown). Furthermore, the minimal inhibitory concentrations (MIC) of the compounds showed significantly high level $(>80 \%)$ antimicrobial activity and their MIC values were determined against the test microorganism, where the high inhibition activity was detected (Table 1 , in brackets).

Table 1. Most relevant antimicrobial activity of $O$-benzyl derivatives expressed as inhibitory effect (\%) and MIC values (in brackets).

\begin{tabular}{|c|c|c|c|c|c|c|c|}
\hline \multirow[b]{3}{*}{ Analogue } & \multirow[b]{3}{*}{ Conc. $(\mu \mathrm{g} / \mathrm{mL})$} & \multicolumn{4}{|c|}{ Inhibitory Effect (\%) \pm RSD (\%) } & & \\
\hline & & \multicolumn{2}{|c|}{ Gram Positive } & \multicolumn{2}{|c|}{ Gram Negative } & \multicolumn{2}{|c|}{ Yeast } \\
\hline & & $\begin{array}{l}\text { B. subtilis } \\
\text { SZMC0209 }\end{array}$ & $\begin{array}{c}\text { S. aureus } \\
\text { SZMC14611 }\end{array}$ & $\begin{array}{c}\text { E. coli } \\
\text { SZMC6271 }\end{array}$ & $\begin{array}{l}\text { P. aeruginosa } \\
\text { SZMC23290 }\end{array}$ & $\begin{array}{l}\text { C. albicans } \\
\text { SZMC1533 }\end{array}$ & $\begin{array}{c}\text { C. krusei } \\
\text { SZMC1352 }\end{array}$ \\
\hline \multirow[t]{2}{*}{ Nystatin } & 100 & - & - & - & - & $\begin{array}{c}93.38 \pm 2.13 \\
(100 \mu \mathrm{g} / \mathrm{mL})\end{array}$ & $\begin{array}{c}92.01 \pm 3.64 \\
(100 \mu \mathrm{g} / \mathrm{mL})\end{array}$ \\
\hline & 10 & - & - & - & - & $92.88 \pm 10.18$ & $58.00 \pm 9.21$ \\
\hline \multirow[t]{2}{*}{ Ampicillin } & 100 & $\begin{array}{c}95.22 \pm 8.40 \\
(<0.78 \mu \mathrm{g} / \mathrm{mL})\end{array}$ & $\begin{array}{c}81.88 \pm 8.99 \\
(<0.78 \mu \mathrm{g} / \mathrm{mL}) \\
\end{array}$ & $\begin{array}{c}94.07 \pm 3.61 \\
(100 \mu \mathrm{g} / \mathrm{mL}) \\
\end{array}$ & $29.03 \pm 2.06$ & - & - \\
\hline & 10 & $93.00 \pm 3.20$ & $70.37 \pm 6.15$ & $89.37 \pm 0.39$ & - & - & - \\
\hline \multirow{2}{*}{3} & 100 & $\begin{array}{c}97.60 \pm 6.42 \\
(100 \mu \mathrm{g} / \mathrm{mL})\end{array}$ & $57.57 \pm 9.93$ & - & $49.10 \pm 7.52$ & - & - \\
\hline & 10 & $59.58 \pm 8.06$ & - & - & - & - & - \\
\hline \multirow{2}{*}{$5 a$} & 100 & $\begin{array}{l}92.82 \pm 4.69 \\
(25 \mu \mathrm{g} / \mathrm{mL})\end{array}$ & $\begin{array}{l}80.07 \pm 2.21 \\
(50 \mu \mathrm{g} / \mathrm{mL})\end{array}$ & - & $54.06 \pm 9.08$ & $\begin{array}{c}91.56 \pm 1.27 \\
(>100 \mu \mathrm{g} / \mathrm{mL})\end{array}$ & $\begin{array}{c}94.88 \pm 2.18 \\
(100 \mu \mathrm{g} / \mathrm{mL})\end{array}$ \\
\hline & 10 & $48.25 \pm 6.16$ & - & - & - & - & - \\
\hline \multirow[t]{2}{*}{$5 b$} & 100 & $\begin{array}{l}86.35 \pm 1.88 \\
(50 \mu \mathrm{g} / \mathrm{mL})\end{array}$ & $71.48 \pm 1.28$ & - & $46.66 \pm 1.37$ & $\begin{array}{c}92.88 \pm 2.63 \\
(>100 \mu \mathrm{g} / \mathrm{mL})\end{array}$ & $93.62 \pm 0.80$ \\
\hline & 10 & $23.23 \pm 3.15$ & - & - & - & - & - \\
\hline \multirow[t]{2}{*}{$7 a$} & 100 & $\begin{array}{l}81.51 \pm 4.73 \\
(50 \mu \mathrm{g} / \mathrm{mL})\end{array}$ & $70.66 \pm 0.91$ & - & - & $\begin{array}{c}87.90 \pm 10.46 \\
(>100 \mu \mathrm{g} / \mathrm{mL})\end{array}$ & - \\
\hline & 10 & - & - & - & - & - & - \\
\hline \multirow[t]{2}{*}{$7 b$} & 100 & $\begin{array}{l}95.34 \pm 4.81 \\
(50 \mu \mathrm{g} / \mathrm{mL})\end{array}$ & $\begin{array}{c}92.34 \pm 1.32 \\
(100 \mu \mathrm{g} / \mathrm{mL}) \\
\end{array}$ & - & $41.59 \pm 3.53$ & - & - \\
\hline & 10 & $50.00 \pm 7.21$ & - & - & - & - & - \\
\hline \multirow[t]{2}{*}{10} & 100 & $\begin{array}{c}95.16 \pm 2.81 \\
(100 \mu \mathrm{g} / \mathrm{mL}) \\
\end{array}$ & $\begin{array}{c}90.71 \pm 3.27 \\
(100 \mu \mathrm{g} / \mathrm{mL})\end{array}$ & - & $50.87 \pm 9.72$ & $\begin{array}{c}95.91 \pm 16.31 \\
(>100 \mu \mathrm{g} / \mathrm{mL})\end{array}$ & - \\
\hline & 10 & $55.43 \pm 15.48$ & - & - & $44.05 \pm 7.57$ & - & - \\
\hline \multirow{2}{*}{$12 a$} & 100 & $\begin{array}{l}95.16 \pm 6.46 \\
(100 \mu \mathrm{g} / \mathrm{mL})\end{array}$ & - & - & $70.85 \pm 6.49$ & $\begin{array}{c}95.83 \pm 11.18 \\
(>100 \mu \mathrm{g} / \mathrm{mL})\end{array}$ & - \\
\hline & 10 & $73.41 \pm 5.45$ & - & - & $47.81 \pm 7.92$ & - & - \\
\hline \multirow[t]{2}{*}{$12 b$} & 100 & $\begin{array}{c}91.84 \pm 6.01 \\
(100 \mu \mathrm{g} / \mathrm{mL}) \\
\end{array}$ & $\begin{array}{c}83.11 \pm 2.61 \\
(100 \mu \mathrm{g} / \mathrm{mL})\end{array}$ & $50.07 \pm 10.97$ & $75.84 \pm 7.14$ & $\begin{array}{c}94.50 \pm 0.97 \\
(>100 \mu \mathrm{g} / \mathrm{mL})\end{array}$ & $67.59 \pm 16.45$ \\
\hline & 10 & $32.17 \pm 11.19$ & - & - & $58.24 \pm 4.20$ & - & - \\
\hline \multirow[t]{2}{*}{$14 a$} & 100 & $\begin{array}{c}92.67 \pm 3.90 \\
(100 \mu \mathrm{g} / \mathrm{mL})\end{array}$ & $\begin{array}{c}82.35 \pm 3.19 \\
(100 \mu \mathrm{g} / \mathrm{mL})\end{array}$ & - & $52.97 \pm 7.47$ & - & - \\
\hline & 10 & - & - & - & $44.00 \pm 1.32$ & - & - \\
\hline \multirow[t]{2}{*}{$20 a$} & 100 & $\begin{array}{c}84.57 \pm 3.18 \\
(6.25 \mu \mathrm{g} / \mathrm{mL})\end{array}$ & $70.13 \pm 0.90$ & - & - & $\begin{array}{c}91.35 \pm 1.07 \\
(>100 \mu \mathrm{g} / \mathrm{mL})\end{array}$ & - \\
\hline & 10 & $89.70 \pm 1.32$ & $65.81 \pm 0.51$ & - & - & - & - \\
\hline \multirow{2}{*}{$20 \mathrm{~b}$} & 100 & $78.34 \pm 2.51$ & $69.49 \pm 0.57$ & - & - & $\begin{array}{c}90.74 \pm 2.90 \\
(>100 \mu \mathrm{g} / \mathrm{mL})\end{array}$ & $79.88 \pm 3.39$ \\
\hline & 10 & $78.43 \pm 5.39$ & $61.84 \pm 0.27$ & - & - & $80.54 \pm 17.23$ & - \\
\hline
\end{tabular}


Table 1. Cont.

\begin{tabular}{|c|c|c|c|c|c|c|c|}
\hline \multicolumn{8}{|c|}{ Inhibitory Effect (\%) \pm RSD (\%) } \\
\hline \multirow[b]{2}{*}{ Analogue } & \multirow[b]{2}{*}{ Conc. $(\mu \mathrm{g} / \mathrm{mL})$} & \multicolumn{2}{|c|}{ Gram Positive } & \multicolumn{2}{|c|}{ Gram Negative } & \multicolumn{2}{|c|}{ Yeast } \\
\hline & & $\begin{array}{l}\text { B. subtilis } \\
\text { SZMC0209 }\end{array}$ & $\begin{array}{c}\text { S. aureus } \\
\text { SZMC14611 }\end{array}$ & $\begin{array}{c}\text { E. coli } \\
\text { SZMC6271 }\end{array}$ & $\begin{array}{c}\text { P. aeruginosa } \\
\text { SZMC23290 }\end{array}$ & $\begin{array}{l}\text { C. albicans } \\
\text { SZMC1533 }\end{array}$ & $\begin{array}{c}\text { C. krusei } \\
\text { SZMC1352 }\end{array}$ \\
\hline \multirow[b]{2}{*}{$22 a$} & 100 & $83.44 \pm 20.97$ & $76.39 \pm 1.13$ & - & - & - & - \\
\hline & 10 & $\begin{array}{l}81.63 \pm 1.22 \\
(25 \mu \mathrm{g} / \mathrm{mL})\end{array}$ & $70.02 \pm 1.01$ & - & - & - & - \\
\hline \multirow[t]{2}{*}{$22 b$} & 100 & $\begin{array}{c}78.43 \pm 10.14 \\
(<0.78 \mu \mathrm{g} / \mathrm{mL})\end{array}$ & $60.32 \pm 1.11$ & - & - & $\begin{array}{c}81.97 \pm 4.00 \\
(>100 \mu \mathrm{g} / \mathrm{mL})\end{array}$ & - \\
\hline & 10 & $81.01 \pm 1.08$ & $62.77 \pm 0.27$ & - & - & $61.02 \pm 6.51$ & - \\
\hline \multirow{2}{*}{$23 a$} & 100 & $\begin{array}{c}73.83 \pm 4.14 \\
(<0.78 \mu \mathrm{g} / \mathrm{mL})\end{array}$ & $73.99 \pm 5.15$ & - & $47.92 \pm 1.67$ & - & - \\
\hline & 10 & $83.29 \pm 5.94$ & - & - & $47.78 \pm 3.40$ & - & - \\
\hline \multirow{2}{*}{$23 b$} & 100 & $75.64 \pm 0.21$ & $71.95 \pm 4.38$ & - & $46.03 \pm 2.10$ & - & - \\
\hline & 10 & $77.54 \pm 5.94$ & - & - & $42.22 \pm 1.49$ & - & - \\
\hline \multirow{2}{*}{$25 a$} & 100 & $78.96 \pm 0.88$ & - & - & - & - & - \\
\hline & 10 & - & - & - & - & - & - \\
\hline \multirow{2}{*}{$27 a$} & 100 & $71.13 \pm 4.78$ & - & - & $43.48 \pm 3.42$ & - & - \\
\hline & 10 & - & - & - & $38.95 \pm 9.32$ & - & - \\
\hline \multirow{2}{*}{$27 \mathrm{~b}$} & 100 & - & - & - & $34.19 \pm 6.00$ & $\begin{array}{c}80.58 \pm 12.34 \\
(>100 \mu \mathrm{g} / \mathrm{mL})\end{array}$ & - \\
\hline & 10 & - & - & - & $33.16 \pm 8.01$ & - & - \\
\hline \multirow{2}{*}{$31 \mathrm{a}$} & 100 & $\begin{array}{c}95.13 \pm 9.21 \\
(100 \mu \mathrm{g} / \mathrm{mL})\end{array}$ & $\begin{array}{c}82.58 \pm 10.08 \\
(>100 \mu \mathrm{g} / \mathrm{mL})\end{array}$ & - & $48.38 \pm 1.94$ & - & - \\
\hline & 10 & $12.76 \pm 9.95$ & - & - & $32.10 \pm 3.98$ & - & - \\
\hline \multirow[t]{2}{*}{$31 b$} & 100 & $\begin{array}{l}93.89 \pm 5.51 \\
(21 \mu \mathrm{g} / \mathrm{mL})\end{array}$ & $\begin{array}{l}86.85 \pm 4.00 \\
(50 \mu \mathrm{g} / \mathrm{mL})\end{array}$ & - & $53.31 \pm 4.84$ & $\begin{array}{c}95.21 \pm 3.59 \\
(100 \mu \mathrm{g} / \mathrm{mL})\end{array}$ & - \\
\hline & 10 & $47.83 \pm 9.92$ & - & - & $47.81 \pm 6.60$ & - & - \\
\hline \multirow[t]{2}{*}{$39 a$} & 100 & $\begin{array}{c}79.38 \pm 4.19 \\
(3.13 \mu \mathrm{g} / \mathrm{mL})\end{array}$ & $63.47 \pm 4.90$ & - & - & $\begin{array}{c}88.22 \pm 3.96 \\
(>100 \mu \mathrm{g} / \mathrm{mL})\end{array}$ & - \\
\hline & 10 & $82.73 \pm 0.52$ & $69.84 \pm 0.00$ & - & - & - & - \\
\hline \multirow[t]{2}{*}{$39 \mathrm{~b}$} & 100 & $\begin{array}{c}87.80 \pm 7.04 \\
(1.56 \mu \mathrm{g} / \mathrm{mL})\end{array}$ & $\begin{array}{c}79.66 \pm 2.59 \\
(3.13 \mu \mathrm{g} / \mathrm{mL})\end{array}$ & - & $48.09 \pm 1.38$ & $\begin{array}{c}90.89 \pm 13.31 \\
(>100 \mu \mathrm{g} / \mathrm{mL})\end{array}$ & $\begin{array}{c}91.08 \pm 4.90 \\
(>100 \mu \mathrm{g} / \mathrm{mL})\end{array}$ \\
\hline & 10 & $92.94 \pm 1.46$ & $83.69 \pm 38.18$ & - & $33.59 \pm 6.43$ & $85.10 \pm 9.56$ & - \\
\hline \multirow{2}{*}{$40 a$} & 100 & $72.88 \pm 1.68$ & $68.26 \pm 1.66$ & - & $37.66 \pm 2.39$ & - & - \\
\hline & 10 & $55.43 \pm 5.07$ & - & - & $38.89 \pm 1.13$ & - & - \\
\hline \multirow{2}{*}{$40 \mathrm{~b}$} & 100 & $71.39 \pm 3.84$ & $69.37 \pm 1.44$ & - & $42.13 \pm 2.25$ & - & - \\
\hline & 10 & $65.82 \pm 4.56$ & - & - & $39.58 \pm 0.73$ & - & - \\
\hline \multirow{2}{*}{$42 a$} & 100 & $69.05 \pm 10.02$ & - & - & - & - & - \\
\hline & 10 & $51.75 \pm 11.13$ & - & - & - & - & - \\
\hline \multirow{2}{*}{$42 b$} & 100 & $\begin{array}{c}86.62 \pm 8.48 \\
(>100 \mu \mathrm{g} / \mathrm{mL})\end{array}$ & $66.22 \pm 4.03$ & - & - & - & - \\
\hline & 10 & $43.95 \pm 5.65$ & - & - & - & - & - \\
\hline \multirow[t]{2}{*}{$43 b$} & 100 & - & - & - & - & $\begin{array}{c}80.90 \pm 4.76 \\
(>100 \mu \mathrm{g} / \mathrm{mL})\end{array}$ & - \\
\hline & 10 & - & - & - & - & - & - \\
\hline
\end{tabular}




\section{Discussion}

\subsection{Antimicrobial Activity}

The MIC values of significant O-benzyl derivatives ( $\mathrm{I} \%>80 \%$ ) obtained against the tested microorganisms are presented in Table 1 . The strongest antifungal activity was shown by compound 22b, 23a (di O-benzyl aminotriols) at a concentration of $0.78 \mu \mathrm{g} / \mathrm{mL}$, they were as same as the reference drug ampicillin $(0.78 \mu \mathrm{g} / \mathrm{mL})$. Another di O-benzyl aminotriols 20a and 39a-b were effective against B. subtilis below than $10 \mu \mathrm{g} / \mathrm{mL}$ of MIC values. Moreover, O-benzyl aminotriols $5 \mathbf{a}-\mathbf{b}, \mathbf{7} \mathbf{a}-\mathbf{b}, \mathbf{3 1} \mathbf{b}$ together with imidazolesubstituted di $O$-benzyl aminotriol 22a showed lower activity against $B$. subtilis with MIC values in the range between 20 and $50 \mu \mathrm{g} / \mathrm{mL}$. The weak effect on $B$. subtilis was observed for compounds 3, 10, 12a-b, 14a, 31a, 42a (MIC $\geq 100 \mu \mathrm{g} / \mathrm{mL}$ ).

Growth inhibition of S. aureus was observed at the concentration of $50 \mu \mathrm{g} / \mathrm{mL}$ of $O$ benzyl aminodiols 5a and 31a. Imidazole-substituted di $O$-benzyl aminotriol $39 \mathbf{b}$ exhibited relatively high antibacterial potency against $S$. aureus at the MIC values of $3.13 \mu \mathrm{g} / \mathrm{mL}$, whereas derivatives $\mathbf{7 b}, \mathbf{1 0}, \mathbf{1 2} \mathbf{b}$, and $\mathbf{1 4} \mathbf{a}$ was less active against $S$. aureus and inhibited bacterial growth at the concentration of $100 \mu \mathrm{g} / \mathrm{mL}$. The MICs of standard drug ampicillin for the $S$. aureus were $0.78 \mu \mathrm{g} / \mathrm{mL}$.

On the other hand, regarding MIC for pathogenic fungi, $O$-benzyl derivatives showed poor activity against all the tested fungal strains, which obtained by the MIC values against C. albicans and C. krusei (>100 $\mu \mathrm{g} / \mathrm{mL})$.

As shown in Table 1, $N$-benzyl and imidazole-substituted $O$-benzyl derivatives showed significant inhibitory activity against Gram-positive bacteria B. subtilis and $S$. aureus. Di-O-benzyl-substituted derivatives $(20,22-23,39-40)$ exerted bactericidal activities against the bacterial species of $B$. subtilis and S. aureus at low concentrations $(10 \mu \mathrm{M})$. Only 12a-b showed significant effect against Gram-negative bacterium P. aeruginosa as well as a moderate effect against $E$. coli $(\mathbf{1 2 b})$. Other derivatives possessed moderate antibacterial activity against $P$. aeruginosa. Three di-O-benzyl derivatives $(\mathbf{2 0 b}, \mathbf{2 2} \mathbf{b}, \mathbf{3 9} \mathbf{b})$ were highly effective against both $C$. albicans and $C$. krusei. Furthermore, $O$-benzyl derivatives $27 \mathbf{b}$ and $\mathbf{4 3 b}$ were found to exhibit marked growth inhibition against $C$. albicans. N-Dibenzylsubstituted $O$-benzyl derivatives were found to be weakly active or inactive against all tested strains.

The obtained results showed that all synthetic derivatives proved to be more active against Gram-positive than against Gram-negative bacteria. O-benzyl derivatives that contain $N$-benzyl and imidazole substitution were the most active compounds against Gram-positive bacteria and had moderate antimicrobial effect against the P. aeruginosa (Gram-negative) strain. The mechanism of bactericidal action of heterocycles containing the imidazole ring is thought to be due to disruption of intermolecular interactions in the cell membrane. This can cause dissociation of cellular membrane lipid bilayers, which compromises cellular permeability controls and induces leakage of cellular contents [89].

Regarding the yeasts, $\mathrm{N}$-benzyl- and imidazole-substituted $\mathrm{O}$-benzyl derivatives were also found to be the most active compounds against $C$. albicans. The imidazole derivatives can inhibit the transformation of blastospores of $C$. albicans into the invasive mycelial form [90]. In addition, the preliminary in vitro antifungal screening indicated that $S$ isomers showed better potency compared to $R$-isomers against $C$. albicans. Since the widely accepted primary effect of imidazoles is the inhibition of cytochrome P450-mediated 14asterol demethylase of the ergosterol precursor lanosterol from C. albians [91]. This enzyme with strict substrate requirements interacted differentially with the stereoisomers of $O$ benzyl derivatives, therefore the affinity of O-benzyl derivatives for cytochrome P-450 enzymes involved in steroid synthesis is highly dependent on the stereochemistry of the entire molecule.

The results obtained showed that the tested O-benzyl derivatives that contain $\mathrm{N}$ dibenzyl substituents have no antibacterial or antifungal activity against any of the tested pathogenic species of bacteria and fungi. The steric hindrance of the substituents, which prevents the destruction of normal permeability, might be the reason for the low antimi- 
crobial and antifungal activity of the $\mathrm{N}$-dibenzyl-substituted derivatives. Therefore, the inactivity of $\mathrm{N}$-dibenzyl derivatives observed in the present study can be due to the mode of substitution.

\subsection{Structure-Activity Relationship}

(i) N,O-dibenzyl aminodiols (5a-b) exhibited significant inhibitory activity against both Gram-positive bacteria (B. subtilis and S. aureus) and Gram-positive bacteria (P. aeruginosa) as well as yeast (C. albicans and C. krusei). Replacing $N$-benzyl substitution by imidazole $(7 \mathbf{a}-\mathbf{b})$ led to the loss of activity against $C$. krusei.

(ii) When the $-\mathrm{CH}_{3}$ group of isopropyl part was changed to $-\mathrm{CH}_{2} \mathrm{OH}$, disappearance on inhibitory activity against $S$. aureus and C. krusei was observed on $\mathrm{N}, \mathrm{O}$-dibenzyl aminodiol containing $R$-isomer (12a) whereas the other stereoisomer (12b) exhibited an additive effect on E. coli. In the case of imidazole $O$-benzyl aminotriols, this route reduced activity on $C$. albicans with $R$-isomer (14a) and totally lost on antifungal effectiveness on the other isomer (14b).

(iii) Benzylation of $-\mathrm{CH}_{2} \mathrm{OH}$ provided di O-benzyl aminotriols. Our tests revealed that the lack of antifungal activity and high potency against positive-Gram bacteria in both $\mathrm{N}$-benzyl (20a-b) and imidazole (24a-b) aminotriols were produced at a low concentration $(10 \mu \mathrm{M})$. This modification probably improves the lipophilic properties that enhanced interactions in the cell membrane. In addition, the synthesized triazole analogues (23a-b) also exhibit marked growth inhibition against Gram-positive bacteria (B. subtilis and $S$. aureus) and Gram-positive bacteria (P. aeruginosa).

(iv) The almost complete loss of antimicrobial activity resulting from the debenzylation on the cyclohexane ring demonstrated with aminotriol derivatives $(\mathbf{2 5 a}-\mathbf{b})$ suggests that the benzyl moiety on cyclohexyl ring is a key element to have satisfactory antimicrobial activity in the case of $\mathrm{N}, \mathrm{O}$-dibenzyl aminotriol whereas they exert markedly selective antibacterial action on $P$. aeruginosa in the case of imidazole $O$-benzyl aminotriol.

(v) In the stereochemistry study of the $\mathrm{OH}$ group on the cyclohexyl ring, aminodiol with $S$-configuration $(\mathbf{2 7} \mathbf{a}-\mathbf{b})$ displayed a potential negative-Gram bacterial effect ( $P$. aeruginosa) while derivatives with $R$-configuration (42a-b) had significant positive-Gram bacterial effect (B. subtilis) whereas the stereochemistry of the O-benzyl substituent on the cyclohexane ring in the aminodiol and aminotriol function has no influence on the antimicrobial effect.

(vi) The available data demonstrated that most of the N-benzyl and imidazolesubstituted $O$-benzyl derivatives exhibited more antimicrobial potency than triazole or $\mathrm{N}, \mathrm{N}$-dibenzyl O-benzyl ones.

(vii) Further, this result indicates that $S$-isomer showed better potency compared to $R$-isomer against fungi.

\section{Materials and Methods}

\subsection{General Methods}

Commercially available compounds were used as obtained from suppliers (Molar Chemicals Ltd., Halásztelek, Hungary; Merck Ltd., Budapest, Hungary and VWR International Ltd., Debrecen, Hungary), while solvents were dried according to standard procedures. Optical rotations were measured in $\mathrm{MeOH}$ at $20^{\circ} \mathrm{C}$, with a Perkin-Elmer 341 polarimeter (PerkinElmer Inc., Shelton, CT, USA). Chromatographic separations and monitoring of reactions were carried out on Merck Kieselgel 60 (Merck Ltd., Budapest, Hungary). Elemental analyses for all prepared compounds were performed on a PerkinElmer 2400 Elemental Analyzer (PerkinElmer Inc., Waltham, MA, USA). GC measurements for direct separation of commercially available enantiomers of isopulegol to determine the enantiomeric purity of starting material 1 were performed on a Chirasil-DEX CB column $(2500 \times 0.25 \mathrm{~mm}$ I.D. $)$ on a Perkin-Elmer Autosystem XL GC equipped with a Flame Ionization Detector (Perkin-Elmer Corporation, Norwalk, CT, USA) and a Turbochrom Workstation data system (Perkin-Elmer Corp., Norwalk, CT, USA). Melting points were 
determined on a Kofler apparatus (Nagema, Dresden, Germany) and are uncorrected. ${ }^{1} \mathrm{H}-$ and ${ }^{13} \mathrm{C}$-NMR spectra were recorded on Brucker Avance DRX 500 spectrometer (Bruker Biospin, Karlsruhe, Baden Württemberg, Germany) $\left[500 \mathrm{MHz}\left({ }^{1} \mathrm{H}\right)\right.$ and $125 \mathrm{MHz}\left({ }^{13} \mathrm{C}\right)$, $\delta=0$ (TMS)]. Chemical shifts are expressed in ppm ( $\delta$ ) relative to TMS as the internal reference. $J$ values are given by $\mathrm{Hz}$.

(-)-Isopulegol (1) is commercially available from Merck Co with $e e=95 \%,\left([\alpha]_{\mathrm{D}}^{20}=-22.0\right.$, neat $)$ and its enatimomer $(+)-\mathbf{1}\left(e e=90 \%,\left[[\alpha]_{\mathrm{D}}^{20}=+22.0\right.\right.$, neat $) .(+)$-Neoisopulegol $(2)\left([\alpha]_{\mathrm{D}}^{20}=+28.7\right.$, $\left.\mathrm{c}=17.2, \mathrm{CHCl}_{3}\right)$ and its enatimomer $(-)-2\left([\alpha]_{\mathrm{D}}^{20}=-22.2, \mathrm{c}=2.0, \mathrm{CHCl}_{3}\right)$ were synthesized from $(-)-\mathbf{1}$ and its isomer $(+)-\mathbf{1}$ following a reported procedure, respectively [71]. Diol 17, epoxide $\mathbf{4 4}$ [72] as well as compounds $\mathbf{2 9}, \mathbf{3 3}$, and $\mathbf{3 7 a - b}$ [68] were prepared according to literature procedures. All spectroscopic data were similar to those described therein. Since any of the applied transformations do not reach all the four chiral centers at the same time, giving rise to racemization, rather only the formation of the prescribed and isolated diastereoisomers, we believe that the enantiomer purity of the prepared compounds can be defined as $e e \geq$ $95 \%$ (commercial (-)-isopulegol). ${ }^{1} \mathrm{H},{ }^{13} \mathrm{C}, \mathrm{HSQC}, \mathrm{HMBC}$ and NOESY NMR spectra of new compounds and GC chromatograms of isopulegol enantiomers are available in Supplementary Materials.

\subsection{Experimental Section and Compound Characterisations}

4.2.1. (S)-2-((1R,2R,4R)-2-Hydroxy-4-methylcyclohexyl)propane-1,2-diol (45)

Compound $44(0.60 \mathrm{mmol})$ was treated with DMSO $(3.0 \mathrm{~mL})$ and $3 \mathrm{M} \mathrm{NaOH}(3.0 \mathrm{~mL})$. The resulting homogenous solution was stirred at $80{ }^{\circ} \mathrm{C}$ for $2 \mathrm{~h}$. After being cooled to room temperature, EtOAc $(20 \mathrm{~mL})$ was added, and the aqueous layer was washed with EtOAc $(3 \times 20 \mathrm{~mL})$. The combined organic layers were dried over $\mathrm{Na}_{2} \mathrm{SO}_{4}$, filtered, and concentrated in vacuo. The crude material was purified by column chromatography on silica gel ( $n$-hexane:EtOAc $=1: 4)$ to provide compound 45.

Yield: $76 \%$, colorless oil. $[\alpha]_{\mathrm{D}}^{20}=+14.0(\mathrm{c} 0.22, \mathrm{MeOH}) .{ }^{1} \mathrm{H}$ NMR $\left(500 \mathrm{MHz}, \mathrm{CDCl}_{3}\right)$ : $\delta=0.88(3 \mathrm{H}, \mathrm{d}, J=6.2 \mathrm{~Hz}), 0.91-0.97(1 \mathrm{H}, \mathrm{m}), 1.10-1.16(1 \mathrm{H}, \mathrm{m}), 1.25(3 \mathrm{H}, \mathrm{s}), 1.35-1.39$ $(1 \mathrm{H}, \mathrm{m}), 1.49-1.53(1 \mathrm{H}, \mathrm{m}), 1.62-1.70(1 \mathrm{H}, \mathrm{m}), 1.76-1.85(3 \mathrm{H}, \mathrm{m}), 3.23(2 \mathrm{H}, \mathrm{brs}), 3.29(1 \mathrm{H}, \mathrm{d}$, $J=11.1 \mathrm{~Hz}), 3.63(1 \mathrm{H}, \mathrm{d}, J=11.1 \mathrm{~Hz}), 4.38(1 \mathrm{H}, \mathrm{s}) .{ }^{13} \mathrm{C} \mathrm{NMR}\left(125 \mathrm{MHz}, \mathrm{CDCl}_{3}\right): \delta=21.4$, 22.3, 25.0, 25.9, 35.2, 42.8, 48.9, 67.0, 67.3, 74.4. Found: C, 63.83; H, 10.69. Anal. Calcd for $\mathrm{C}_{10} \mathrm{H}_{20} \mathrm{O}_{3}: \mathrm{C}, 63.80 ; \mathrm{H}, 10.71$.

\subsubsection{2-((1S,2S,4R)-2-(Benzyloxy)-4-methylcyclohexyl)prop-2-en-1-ol (10)}

To a solution of $t$ - $\mathrm{BuOOH}\left(70 \%\right.$ purity in $\left.\mathrm{H}_{2} \mathrm{O}, 32.80 \mathrm{mmol}\right)$ in $\mathrm{CH}_{2} \mathrm{Cl}_{2}(50 \mathrm{~mL})$, dried briefly $\left(\mathrm{Na}_{2} \mathrm{SO}_{4}\right)$, was added finely powdered $\mathrm{SeO}_{2}(1.96 \mathrm{mmol})$ followed by 30 minutes by the addition of $3(8.20 \mathrm{mmol})$. After stirring for $20 \mathrm{~h}$ at $25^{\circ} \mathrm{C}$, saturated $\mathrm{NaHCO}_{3}$ solution (50 mL) was added, then $\mathrm{CH}_{2} \mathrm{Cl}_{2}$ phases were separated and the aqueous phase was extracted with $\mathrm{CH}_{2} \mathrm{Cl}_{2}(3 \times 50 \mathrm{~mL})$. The organic layer was dried $\left(\mathrm{Na}_{2} \mathrm{SO}_{4}\right)$ and concentrated in vacuo to afford colorless oil, which was added at $0{ }^{\circ} \mathrm{C}$ to a suspension of $\mathrm{NaBH}_{4}(24.60 \mathrm{mmol})$ in dry MeOH $(50 \mathrm{~mL})$. The reaction mixture was stirred for $2 \mathrm{~h}$ at $0{ }^{\circ} \mathrm{C}$ while the reaction progress was monitored by TLC. When the reaction was complete, the mixture was poured into brine $(100 \mathrm{~mL})$ and the product was extracted with $\mathrm{CH}_{2} \mathrm{Cl}_{2}$ $(3 \times 100 \mathrm{~mL})$. The combined extracts were washed with water and dried over anhydrous $\mathrm{Na}_{2} \mathrm{SO}_{4}$. The solvent was evaporated in vacuo. The crude product was purified by column chromatography on silica gel using $n$-hexane:EtOAc $=4: 1$.

Yield: $27 \%$, colorless oil. $[\alpha]_{\mathrm{D}}^{20}=+29.0(\mathrm{c} 0.27, \mathrm{MeOH}) .{ }^{1} \mathrm{H} \mathrm{NMR}\left(500 \mathrm{MHz}, \mathrm{CDCl}_{3}\right)$ : $\delta=0.89(3 \mathrm{H}, \mathrm{d}, J=6.4 \mathrm{~Hz}), 0.94-1.07(2 \mathrm{H}, \mathrm{m}), 1.50-1.55(1 \mathrm{H}, \mathrm{m}), 1.76-1.80(2 \mathrm{H}, \mathrm{m}), 1.87-1.95$ $(1 \mathrm{H}, \mathrm{m}), 2.07-2.11(1 \mathrm{H}, \mathrm{m}), 2.24(1 \mathrm{H}, \mathrm{d}, J=13.0 \mathrm{~Hz}), 2.67(1 \mathrm{H}, \mathrm{t}, J=5.4 \mathrm{~Hz}), 3.71(1 \mathrm{H}$, $\mathrm{d}, J=2.4 \mathrm{~Hz}), 3.94(1 \mathrm{H}, \mathrm{dd}, J=12.7,5.8 \mathrm{~Hz}), 4.06(1 \mathrm{H}, \mathrm{dd}, J=12.7,4.1 \mathrm{~Hz}), 4.34(1 \mathrm{H}, \mathrm{d}$, $J=11.6 \mathrm{~Hz}), 4.60(1 \mathrm{H}, \mathrm{d}, J=11.7 \mathrm{~Hz}), 4.96(1 \mathrm{H}, \mathrm{s}), 5.07(1 \mathrm{H}, \mathrm{d}, J=1.0 \mathrm{~Hz}), 7.25-7.32(5 \mathrm{H}, \mathrm{m})$. ${ }^{13} \mathrm{C}$ NMR $\left(125 \mathrm{MHz}, \mathrm{CDCl}_{3}\right): \delta=22.5,25.0,26.0,35.0,37.5,46.6,65.2,70.6,77.3,113.2,127.7$, $127.9,128.4,138.4$, 151.0. Found: $\mathrm{C}, 78.40 ; \mathrm{H}, 9.33$. Anal. Calcd for $\mathrm{C}_{17} \mathrm{H}_{24} \mathrm{O}_{2}$ : C, 78.42; $\mathrm{H}$, 9.29. 


\subsubsection{General Procedure for Benzylation}

A suspension of $\mathrm{NaH}(60 \%$ purity, $6.6 \mathrm{mmol})$ in dry THF $(10 \mathrm{~mL})$ was added to a solution of alcohol $(6.6 \mathrm{mmol})$ in dry THF $(20 \mathrm{~mL})$. The reaction mixture was stirred at $25^{\circ} \mathrm{C}$ for $30 \mathrm{~min}$ before benzyl bromide $(9.9-19.8 \mathrm{mmol})$ and $\mathrm{KI}(6.6 \mathrm{mmol})$ were added to the mixture. Stirring was continued for $12-24 \mathrm{~h}$ at $25-60{ }^{\circ} \mathrm{C}$. When the reaction was complete, the mixture was poured into saturated $\mathrm{NH}_{4} \mathrm{Cl}$ solution $(30 \mathrm{~mL})$ and extracted with EtOAc $(3 \times 50 \mathrm{~mL})$. The combined organic phase was dried over anhydrous $\mathrm{Na}_{2} \mathrm{SO}_{4}$. The solvent was evaporated in vacuo and the crude product was purified by column chromatography on silica gel to provide 3 or $\mathbf{1 8} \mathbf{a}-\mathbf{b}$, respectively.

((((1S,2S,5R)-5-Methyl-2-(prop-1-en-2-yl)cyclohexyl)oxy)methyl)benzene (3)

Prepared with 2 and benzyl bromide $(9.9 \mathrm{mmol})$ at reflux for $12 \mathrm{~h}$ and eluted by $n$-hexane:EtOAc $=19: 1$. Yield: $63 \%$, colorless oil. $[\alpha]_{\mathrm{D}}^{20}=+24.0(\mathrm{c} 0.28, \mathrm{MeOH}) .{ }^{1} \mathrm{H} \mathrm{NMR}$ $\left(500 \mathrm{MHz}, \mathrm{CDCl}_{3}\right): \delta=0.87(3 \mathrm{H}, \mathrm{d}, J=6.4 \mathrm{~Hz}), 0.86-0.89(2 \mathrm{H}, \mathrm{m}), 0.92-1.00(2 \mathrm{H}, \mathrm{m}), 1.25-$ $1.31(2 \mathrm{H}, \mathrm{m}), 1.51-1.54(1 \mathrm{H}, \mathrm{m}), 1.73(3 \mathrm{H}, \mathrm{s}), 1.74-1.80(2 \mathrm{H}, \mathrm{m}), 1.85-1.95(2 \mathrm{H}, \mathrm{m}), 2.01-2.06$ $(1 \mathrm{H}, \mathrm{m}), 3.75(1 \mathrm{H}, \mathrm{d}, J=1.6 \mathrm{~Hz}), 4.38(1 \mathrm{H}, \mathrm{d}, J=12.1 \mathrm{~Hz}), 4.56(1 \mathrm{H}, \mathrm{d}, J=12.1 \mathrm{~Hz}), 4.77(1 \mathrm{H}$, $\mathrm{d}, J=0.5 \mathrm{~Hz}), 4.80(1 \mathrm{H}, \mathrm{s}), 7.21-7.32(5 \mathrm{H}, \mathrm{m}) .{ }^{13} \mathrm{C} \mathrm{NMR}\left(125 \mathrm{MHz}, \mathrm{CDCl}_{3}\right): \delta=22.4,22.5$, $22.8,25.2$, 26.3, 35.2, 38.6, 48.6, 70.8, 76.1, 110.5, 127.2, 127.5, 128.2, 139.8, 148.0. Found: C, 83.50; $\mathrm{H}, 9.93$. Anal. Calcd for $\mathrm{C}_{17} \mathrm{H}_{24} \mathrm{O}$ : C, 83.55; $\mathrm{H}, 9.90$.

(((2-((1S,2S,4R)-2-(Benzyloxy)-4-methylcyclohexyl)allyl)oxy)methyl)benzene (18a)

Prepared with 17 and benzyl bromide (19.8 mmol) at reflux for $24 \mathrm{~h}$ and eluted by $n$-hexane:EtOAc $=19: 1$. Yield: $56 \%$, colorless oil. $[\alpha]_{\mathrm{D}}^{20}=+20.0($ c $0.25, \mathrm{MeOH}) .{ }^{1} \mathrm{H} \mathrm{NMR}$ $\left(500 \mathrm{MHz}, \mathrm{CDCl}_{3}\right): \delta=0.88(3 \mathrm{H}, \mathrm{d}, J=6.4 \mathrm{~Hz}), 0.94-1.01(2 \mathrm{H}, \mathrm{m}), 1.20-1.30(3 \mathrm{H}, \mathrm{m}), 1.52-$ $1.57(5 \mathrm{H}, \mathrm{m}), 1.75-1.78(2 \mathrm{H}, \mathrm{m}), 1.83-1.91(1 \mathrm{H}, \mathrm{m}), 2.02-2.05(1 \mathrm{H}, \mathrm{m}), 2.13-2.17(1 \mathrm{H}, \mathrm{m}), 3.71$ $(1 \mathrm{H}, \mathrm{s}), 3.89(1 \mathrm{H}, \mathrm{d}, J=12.5 \mathrm{~Hz}), 3.99(1 \mathrm{H}, \mathrm{d}, J=12.5 \mathrm{~Hz}), 4.31(1 \mathrm{H}, \mathrm{d}, J=12.0 \mathrm{~Hz}), 4.38(1 \mathrm{H}$, $\mathrm{d}, J=11.9 \mathrm{~Hz}), 4.46(1 \mathrm{H}, \mathrm{d}, J=12.0 \mathrm{~Hz}), 4.54(1 \mathrm{H}, \mathrm{d}, J=12.0 \mathrm{~Hz}), 5.06(1 \mathrm{H}, \mathrm{s}), 5.14(1 \mathrm{H}, \mathrm{s})$, 7.23-7.36 (10H, m). ${ }^{13} \mathrm{C} \mathrm{NMR}\left(125 \mathrm{MHz}, \mathrm{CDCl}_{3}\right): \delta=22.5,25.2,26.3,35.2,38.2,44.5,70.6$, 72.0, 73.2, 112.9, 127.3, 127.5, 127.6, 127.8, 128.3, 128.5, 147.8. Found: C, 82.27; H, 8.67. Anal. Calcd for $\mathrm{C}_{24} \mathrm{H}_{30} \mathrm{O}_{2}$ : C, 82.24; $\mathrm{H}, 8.63$.

\section{(1S,2S,5R)-2-(3-(Benzyloxy)prop-1-en-2-yl)-5-methylcyclohexanol (18b)}

Prepared with 17 and benzyl bromide $(9.9 \mathrm{mmol})$ at $25^{\circ} \mathrm{C}$ for $12 \mathrm{~h}$ and eluted by $n$-hexane:EtOAc $=9: 1$. Yield: $59 \%$, colorless oil. $[\alpha]_{\mathrm{D}}^{20}=+33.0($ c $0.28, \mathrm{MeOH}) .{ }^{1} \mathrm{H}$ NMR $\left(500 \mathrm{MHz}, \mathrm{CDCl}_{3}\right): \delta=0.88(3 \mathrm{H}, \mathrm{d}, J=6.5 \mathrm{~Hz}), 0.91-1.01(1 \mathrm{H}, \mathrm{m}), 1.13(1 \mathrm{H}, \mathrm{t}, J=12.9 \mathrm{~Hz})$, 1.41-1.47 $(1 \mathrm{H}, \mathrm{m}), 1.62(1 \mathrm{H}, \mathrm{s}), 1.74-1.83(3 \mathrm{H}, \mathrm{m}), 1.90-1.95(1 \mathrm{H}, \mathrm{m}), 2.21(1 \mathrm{H}, \mathrm{d}, J=12.7 \mathrm{~Hz})$, $2.26(1 \mathrm{H}, \mathrm{s}), 3.91(1 \mathrm{H}, \mathrm{d}, J=11.8 \mathrm{~Hz}), 3.96(1 \mathrm{H}, \mathrm{s}), 4.07(1 \mathrm{H}, \mathrm{d}, J=11.7 \mathrm{~Hz}), 4.48(1 \mathrm{H}, \mathrm{d}$, $J=11.9 \mathrm{~Hz}), 4.54(1 \mathrm{H}, \mathrm{d}, J=11.8 \mathrm{~Hz}), 5.06(1 \mathrm{H}, \mathrm{s}), 5.21(1 \mathrm{H}, \mathrm{s}), 7.25-7.36(5 \mathrm{H}, \mathrm{m}) .{ }^{13} \mathrm{C}$ NMR $\left(125 \mathrm{MHz}, \mathrm{CDCl}_{3}\right): \delta=22.4,24.1,25.8,35.0,41.3,45.9,67.7,72.5,72.7,115.2,127.9,128.6$, 138.0, 143.4, 147.8. Found: $\mathrm{C}, 78.45 ; \mathrm{H}, 9.27$. Anal. Calcd for $\mathrm{C}_{17} \mathrm{H}_{24} \mathrm{O}_{2}: \mathrm{C}, 78.42 ; \mathrm{H}, 9.29$.

\subsubsection{General Procedure of Epoxidation}

To the solution of allylic alcohol derivatives $(11.9 \mathrm{mmol})$ in $\mathrm{CH}_{2} \mathrm{Cl}_{2}(50 \mathrm{~mL}), \mathrm{Na}_{2} \mathrm{HPO}_{4} \cdot 12 \mathrm{H}_{2} \mathrm{O}$ ( $35.7 \mathrm{mmol})$ in water $(130 \mathrm{~mL})$ and $m$-CPBA $(70 \%$ purity, $23.8 \mathrm{mmol})$ were added at $0{ }^{\circ} \mathrm{C}$, then the mixture was stirred at room temperature. When the reaction was complete $(2 \mathrm{~h})$, the mixture was separated, and the aqueous phase was extracted with $\mathrm{CH}_{2} \mathrm{Cl}_{2}(100 \mathrm{~mL})$. The organic layer was washed with $5 \% \mathrm{KOH}$ solution $(3 \times 50 \mathrm{~mL})$, dried $\left(\mathrm{Na}_{2} \mathrm{SO}_{4}\right)$ and concentrated in vacuo. The residue was purified by column chromatography on silica gel with an appropriate solvent mixture to afford epoxides.

(R)-2-((1R,2S,4R)-2-(benzyloxy)-4-methylcyclohexyl)-2-methyloxirane (4a)

Prepared with 3 eluted by $n$-hexane:EtOAc $=9: 1$. Yield: $23 \%$, colorless oil. $[\alpha]_{\mathrm{D}}^{20}=+32.0$ (c $0.285, \mathrm{MeOH}) .{ }^{1} \mathrm{H}$ NMR $\left(500 \mathrm{MHz}, \mathrm{CDCl}_{3}\right): \delta=0.87(3 \mathrm{H}, \mathrm{d}, J=6.5 \mathrm{~Hz}), 0.85-0.95(2 \mathrm{H}, \mathrm{m})$, $1.28(3 \mathrm{H}, \mathrm{s}), 1.44-1.56(3 \mathrm{H}, \mathrm{m}), 1.71-1.76(2 \mathrm{H}, \mathrm{m}), 2.06-2.11(1 \mathrm{H}, \mathrm{m}), 2.51(1 \mathrm{H}, \mathrm{d}, J=4.9 \mathrm{~Hz})$, 
$2.73(1 \mathrm{H}, \mathrm{d}, J=4.9 \mathrm{~Hz}), 3.87(1 \mathrm{H}, \mathrm{d}, J=2.1 \mathrm{~Hz}), 4.39(1 \mathrm{H}, \mathrm{d}, J=11.8 \mathrm{~Hz}), 4.62(1 \mathrm{H}, \mathrm{d}$, $J=11.8 \mathrm{~Hz}), 7.25-7.33(5 \mathrm{H}, \mathrm{m}) .{ }^{13} \mathrm{C}$ NMR $\left(125 \mathrm{MHz}, \mathrm{CDCl}_{3}\right): \delta=20.8,22.1,22.4,26.4,34.6$, 37.7, 47.2, 53.6, 58.5, 70.3, 74.9, 127.4, 127.5, 128.4, 139.4. Found: C, 78.47; H 9.33. Anal. Calcd for $\mathrm{C}_{17} \mathrm{H}_{24} \mathrm{O}_{2}$ : C, 78.42; $\mathrm{H}, 9.29$.

(S)-2-((1R,2S,4R)-2-(Benzyloxy)-4-methylcyclohexyl)-2-methyloxirane (4b)

Prepared with 3 eluted by $n$-hexane:EtOAc $=9: 1$. Yield: $47 \%$, colorless oil. $[\alpha]_{\mathrm{D}}^{20}=+88.7$ (c $0.385, \mathrm{MeOH}) .{ }^{1} \mathrm{H}$ NMR $\left(500 \mathrm{MHz}, \mathrm{CDCl}_{3}\right): \delta=0.87(3 \mathrm{H}, \mathrm{d}, J=6.4 \mathrm{~Hz}), 0.84-0.95(2 \mathrm{H}, \mathrm{m})$, 1.19-1.24 (1H, m), $1.33(3 \mathrm{H}, \mathrm{s}), 1.62-1.66(1 \mathrm{H}, \mathrm{m}), 1.77-1.82(3 \mathrm{H}, \mathrm{m}), 2.02-2.07(1 \mathrm{H}, \mathrm{m}), 2.49$ $(1 \mathrm{H}, \mathrm{d}, J=4.9 \mathrm{~Hz}), 2.68(1 \mathrm{H}, \mathrm{d}, J=4.9 \mathrm{~Hz}), 2.72(1 \mathrm{H}, \mathrm{d}, J=2.2 \mathrm{~Hz}), 4.35(1 \mathrm{H}, \mathrm{d}, J=11.7 \mathrm{~Hz})$, $4.60(1 \mathrm{H}, \mathrm{d}, J=11.7 \mathrm{~Hz}), 7.25-7.34(5 \mathrm{H}, \mathrm{m}) .{ }^{13} \mathrm{C} \mathrm{NMR}\left(125 \mathrm{MHz}, \mathrm{CDCl}_{3}\right): \delta=20.5,22.4,23.2$, 26.3, 34.8, 37.6, 53.4, 59.4, 70.1, 75.6, 76.9, 77.1, 127.4, 127.6, 128.4, 139.2. Found: C, 78.40; $\mathrm{H}$ 9.25. Anal. Calcd for $\mathrm{C}_{17} \mathrm{H}_{24} \mathrm{O}_{2}: \mathrm{C}, 78.42 ; \mathrm{H}, 9.29$.

(S)-2-((1R,2S,4R)-2-(Benzyloxy)-4-methylcyclohexyl)-2-((benzyloxy)methyl)oxirane (19a)

Prepared with 18a eluted by $n$-hexane:EtOAc $=9: 1$. Yield: $36 \%$, colorless oil. $[\alpha]_{\mathrm{D}}^{20}=+47.0$ (c $0.25, \mathrm{MeOH}) .{ }^{1} \mathrm{H}$ NMR $\left(500 \mathrm{MHz}, \mathrm{CDCl}_{3}\right): \delta=0.87(3 \mathrm{H}, \mathrm{d}, J=6.4 \mathrm{~Hz}), 0.86-0.97(2 \mathrm{H}$, m), 1.40-1.50 (2H, m), 1.69-1.75 (2H, m), 1.89-1.93 (1H, m), 2.04-2.09 $(1 \mathrm{H}, \mathrm{m}), 2.64(1 \mathrm{H}, \mathrm{d}$, $J=4.7 \mathrm{~Hz}), 2.80(1 \mathrm{H}, \mathrm{d}, J=4.8 \mathrm{~Hz}), 2.87(1 \mathrm{H}, \mathrm{d}, J=11.6 \mathrm{~Hz}), 3.73(1 \mathrm{H}, \mathrm{d}, J=11.6 \mathrm{~Hz}), 3.83(1 \mathrm{H}$, $\mathrm{d}, J=5.4 \mathrm{~Hz}), 4.25(1 \mathrm{H}, \mathrm{d}, J=11.8 \mathrm{~Hz}), 4.40(1 \mathrm{H}, \mathrm{d}, J=12.1 \mathrm{~Hz}), 4.51(1 \mathrm{H}, \mathrm{d}, J=12.0 \mathrm{~Hz}), 4.57$ $(1 \mathrm{H}, \mathrm{d}, J=11.9 \mathrm{~Hz}), 7.24-7.34(10 \mathrm{H}, \mathrm{m}) .{ }^{13} \mathrm{C}$ NMR $\left(125 \mathrm{MHz}, \mathrm{CDCl}_{3}\right): \delta=21.1,22.4,26.4,34.5$, 37.3, 42.4, 48.3, 60.1, 70.1, 71.6, 73.2, 74.3, 127.4, 127.5, 127.7, 127.8, 128.4, 128.5. Found: C, 78.67; $\mathrm{H}$ 8.23. Anal. Calcd for $\mathrm{C}_{24} \mathrm{H}_{30} \mathrm{O}_{3}: \mathrm{C}, 78.65 ; \mathrm{H}, 8.25$.

(R)-2-((1R,2S,4R)-2-(Benzyloxy)-4-methylcyclohexyl)-2-((benzyloxy)methyl)oxirane (19b)

Prepared with 18a eluted by $n$-hexane:EtOAc $=9: 1$. Yield: $36 \%$, colorless oil. $[\alpha]_{\mathrm{D}}^{20}=+54.0$ (c $0.25, \mathrm{MeOH}) .{ }^{1} \mathrm{H} \mathrm{NMR}\left(500 \mathrm{MHz}, \mathrm{CDCl}_{3}\right): \delta=0.86(3 \mathrm{H}, \mathrm{d}, J=6.5 \mathrm{~Hz}), 0.85-0.95(4 \mathrm{H}, \mathrm{m})$, $1.26-1.29(2 \mathrm{H}, \mathrm{m}), 1.50(2 \mathrm{H}, \mathrm{m}), 1.62-1.76(5 \mathrm{H}, \mathrm{m}), 2.01-2.04(1 \mathrm{H}, \mathrm{m}), 2.69(1 \mathrm{H}, \mathrm{d}, J=5.3 \mathrm{~Hz})$, $2.84(1 \mathrm{H}, \mathrm{d}, J=5.4 \mathrm{~Hz}), 3.58(1 \mathrm{H}, \mathrm{d}, J=10.9 \mathrm{~Hz}), 3.70(1 \mathrm{H}, \mathrm{d}, J=11.4 \mathrm{~Hz}), 3.76(1 \mathrm{H}, \mathrm{s}) 4.32(1 \mathrm{H}$, $\mathrm{d}, J=11.6 \mathrm{~Hz}), 4.48(2 \mathrm{H}, \mathrm{s}), 4.54(1 \mathrm{H}, \mathrm{d}, J=11.6 \mathrm{~Hz}), 7.23-7.32(10 \mathrm{H}, \mathrm{m}) .{ }^{13} \mathrm{C}$ NMR $(125 \mathrm{MHz}$, $\left.\mathrm{CDCl}_{3}\right): \delta=22.4,23.1,26.3,34.9,37.7,43.9,48.9,60.8,70.2,71.3,73.5,74.8,127.4,127.7,127.8$, 128.4, 128.4, 128.5, 138.6, 139.3. Found: $\mathrm{C}, 78.62 ; \mathrm{H}$ 8.23. Anal. Calcd for $\mathrm{C}_{24} \mathrm{H}_{30} \mathrm{O}_{3}: \mathrm{C}, 78.65$; $\mathrm{H}, 8.25$.

$(1 R, 2 R, 5 R)-2-((S)-2-(($ Benzyloxy)methyl)oxiran-2-yl)-5-methylcyclohexanol (24a)

Prepared with $18 b$ eluted by $n$-hexane:EtOAc $=4: 1$. Yield: $42 \%$, colorless oil. $[\alpha]_{\mathrm{D}}^{20}=+37.0$ (c $0.275, \mathrm{MeOH}) .{ }^{1} \mathrm{H}$ NMR $\left(500 \mathrm{MHz}, \mathrm{CDCl}_{3}\right): \delta=0.86(3 \mathrm{H}, \mathrm{d}, J=6.4 \mathrm{~Hz}), 0.88-0.96(1 \mathrm{H}, \mathrm{m})$, 1.00-1.06 (1H, m), 1.45-1.49 (1H, m), 1.55-1.59 (2H, m), 1.66-1.1.78 (2H, m), 1.82-1.87 (2H, m), $2.67(1 \mathrm{H}, \mathrm{d}, J=4.6 \mathrm{~Hz}), 2.80(1 \mathrm{H}, \mathrm{d}, J=4.6 \mathrm{~Hz}), 3.22(1 \mathrm{H}, \mathrm{d}, J=10.3 \mathrm{~Hz}), 3.37(1 \mathrm{H}, \mathrm{s}), 3.82(1 \mathrm{H}$, $\mathrm{d}, J=10.3 \mathrm{~Hz}), 4.18(1 \mathrm{H}, \mathrm{s}), 4.53(1 \mathrm{H}, \mathrm{d}, J=11.6 \mathrm{~Hz}), 4.57(1 \mathrm{H}, \mathrm{d}, J=11.8 \mathrm{~Hz}), 7.25-7.37(5 \mathrm{H}$, m). ${ }^{13} \mathrm{C}$ NMR (125 MHz, $\mathrm{CDCl}_{3}$ ): $\delta=22.2,22.3,25.6,34.7,41.7,44.0,50.2,60.6,67.8,72.1,73.7$, 127.9, 128.0, 128.5, 137.1. Found: C, 78.90; H 8.77. Anal. Calcd for $\mathrm{C}_{17} \mathrm{H}_{24} \mathrm{O}_{3}$ : C, 73.88; $\mathrm{H}, 8.75$.

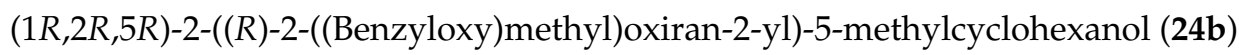

Prepared with $18 b$ eluted by $n$-hexane:EtOAc $=4: 1$. Yield: $15 \%$, colorless oil. $[\alpha]_{\mathrm{D}}^{20}=+24.0$ (c $0.295, \mathrm{MeOH}) .{ }^{1} \mathrm{H}$ NMR $\left(500 \mathrm{MHz}, \mathrm{CDCl}_{3}\right): \delta=0.86(3 \mathrm{H}, \mathrm{d}, J=6.4 \mathrm{~Hz}), 0.88-0.95(1 \mathrm{H}$, $\mathrm{m}), 1.02-1.07(1 \mathrm{H}, \mathrm{m}), 1.47-1.50(1 \mathrm{H}, \mathrm{m}), 1.57(1 \mathrm{H}, \mathrm{s}), 1.59-1.66(2 \mathrm{H}, \mathrm{m}), 1.74-1.77(1 \mathrm{H}, \mathrm{m})$, $1.82-1.88(2 \mathrm{H}, \mathrm{m}), 2.69(1 \mathrm{H}, \mathrm{d}, J=4.6 \mathrm{~Hz}), 2.85(1 \mathrm{H}, \mathrm{d}, J=4.6 \mathrm{~Hz}), 3.24(1 \mathrm{H}, \mathrm{s}), 3.43(1 \mathrm{H}$, $\mathrm{d}, J=10.8 \mathrm{~Hz}), 3.69(1 \mathrm{H}, \mathrm{d}, J=10.9 \mathrm{~Hz}), 4.14(1 \mathrm{H}, \mathrm{s}), 4.53(1 \mathrm{H}, \mathrm{d}, J=11.8 \mathrm{~Hz}), 4.61(1 \mathrm{H}, \mathrm{d}$, $J=11.9 \mathrm{~Hz}), 7.25-7.35(5 \mathrm{H}, \mathrm{m}) .{ }^{13} \mathrm{C} \mathrm{NMR}\left(125 \mathrm{MHz}, \mathrm{CDCl}_{3}\right): \delta=21.9,22.3,25.9,34.9,41.8$, 44.3, 50.7, 66.7, 72.2, 73.8, 128.0, 128.1, 128.7, 137.4. Found: C, 78.85; H 8.74. Anal. Calcd for $\mathrm{C}_{17} \mathrm{H}_{24} \mathrm{O}_{3}$ : C, 73.88; $\mathrm{H}, 8.75$. 
4.2.5. General Procedure for Ring-Opening of Epoxides with Different Amines

A solution of epoxides $(2.9 \mathrm{mmol})$ in $\mathrm{MeCN}(30 \mathrm{~mL})$ was added to the appropriate amines $(5.8 \mathrm{mmol})$ in $\mathrm{MeCN}(10 \mathrm{~mL})$ and $\mathrm{LiClO}_{4}(2.9 \mathrm{mmol})$. The mixture was kept at reflux temperature for 6-20 $\mathrm{h}$. When the reaction was completed (indicated by TLC), the mixture was evaporated to dryness, the residue was again dissolved in water $(15 \mathrm{~mL})$, and then extracted with $\mathrm{CH}_{2} \mathrm{Cl}_{2}(3 \times 50 \mathrm{~mL})$. The combined organic phase was dried $\left(\mathrm{Na}_{2} \mathrm{SO}_{4}\right)$, filtered, and concentrated. The crude product was purified by column chromatography on silica gel with an appropriate solvent mixture, resulting in O-benzyl derivatives, respectively.

(R)-1-(Benzylamino)-2-((1R,2S,4R)-2-(benzyloxy)-4-methylcyclohexyl)propan-2-ol (5a)

Prepared with $4 \mathrm{a}$ with benzylamine at reflux for $20 \mathrm{~h}$ and eluted by $n$-hexane:EtOAc $=1: 1$. Yield: $78 \%$, colorless oil. $[\alpha]_{\mathrm{D}}^{20}=+41.0(\mathrm{c} 0.275, \mathrm{MeOH}) .{ }^{1} \mathrm{H}$ NMR $\left(500 \mathrm{MHz}, \mathrm{CDCl}_{3}\right): \delta=0.87$ $(3 \mathrm{H}, \mathrm{d}, J=6.6 \mathrm{~Hz}), 0.83-0.97(2 \mathrm{H}, \mathrm{m}), 1.17(3 \mathrm{H}, \mathrm{s}), 1.42-1.46(1 \mathrm{H}, \mathrm{m}), 1.62-1.80(4 \mathrm{H}, \mathrm{m}), 2.05-$ $2.09(1 \mathrm{H}, \mathrm{m}), 2.54(1 \mathrm{H}, \mathrm{d}, J=11.6 \mathrm{~Hz}), 2.63(1 \mathrm{H}, \mathrm{d}, J=11.6 \mathrm{~Hz}), 3.71(1 \mathrm{H}, \mathrm{d}, J=13.3 \mathrm{~Hz}), 3.80$ $(1 \mathrm{H}, \mathrm{d}, J=13.3 \mathrm{~Hz}), 3.92(1 \mathrm{H}, \mathrm{d}, J=1.5 \mathrm{~Hz}), 4.13(1 \mathrm{H}, \mathrm{d}, J=11.3 \mathrm{~Hz}), 4.50(1 \mathrm{H}, \mathrm{d}, J=11.2 \mathrm{~Hz})$, 7.23-7.33 (10H, m). ${ }^{13} \mathrm{C} \mathrm{NMR}\left(125 \mathrm{MHz}, \mathrm{CDCl}_{3}\right): \delta=21.2,22.4,24.3,26.1,35.1,37.1,46.6,54.5$, $58.2,69.7,73.8,75.5,127.1,127.8,127.9,128.5,128.6,138.4$, 140.4. Found: C, 78.45; H, 9.07; N, 3.79. Anal. Calcd for $\mathrm{C}_{24} \mathrm{H}_{33} \mathrm{NO}_{2}$ : C, 78.43; $\mathrm{H}, 9.05 ; \mathrm{N}, 3.81$.

\section{(S)-1-(Benzylamino)-2-((1R,2S,4R)-2-(benzyloxy)-4-methylcyclohexyl)propan-2-ol (5b)}

Prepared with 4 with benzylamine at reflux for $20 \mathrm{~h}$ and eluted by $n$-hexane:EtOAc $=1: 1$. Yield: $64 \%$, colorless oil. $[\alpha]_{\mathrm{D}}^{20}=+7.0(\mathrm{c} 0.25, \mathrm{MeOH}) .{ }^{1} \mathrm{H} \mathrm{NMR}\left(500 \mathrm{MHz}, \mathrm{CDCl}_{3}\right): \delta=0.90$ $(3 \mathrm{H}, \mathrm{d}, J=6.4 \mathrm{~Hz}), 0.85-0.93(2 \mathrm{H}, \mathrm{m}), 0.93-1.00(1 \mathrm{H}, \mathrm{m}), 1.02-1.07(1 \mathrm{H}, \mathrm{m}), 1.20-1.29(4 \mathrm{H}, \mathrm{m})$, $1.26(3 \mathrm{H}, \mathrm{s}), 1.53-1.57(1 \mathrm{H}, \mathrm{m}), 1.63-1.71(2 \mathrm{H}, \mathrm{m}), 2.16-2.22(1 \mathrm{H}, \mathrm{m}), 2.58(1 \mathrm{H}, \mathrm{d}, J=12.4 \mathrm{~Hz})$, $2.78(1 \mathrm{H}, \mathrm{d}, J=12.4 \mathrm{~Hz}), 3.53(1 \mathrm{H}, \mathrm{d}, J=13.4 \mathrm{~Hz}), 3.63(1 \mathrm{H}, \mathrm{d}, J=13.4 \mathrm{~Hz}), 4.20(1 \mathrm{H}, \mathrm{s}), 4.37$ $(1 \mathrm{H}, \mathrm{d}, J=10.3 \mathrm{~Hz}), 4.63(1 \mathrm{H}, \mathrm{d}, J=10.4 \mathrm{~Hz}), 7.02-7.04(2 \mathrm{H}, \mathrm{m}), 7.28-7.45(8 \mathrm{H}, \mathrm{m}) .{ }^{13} \mathrm{C}$ NMR $\left(125 \mathrm{MHz}, \mathrm{CDCl}_{3}\right): \delta=22.2,26.2,28.1,29.8,35.0,36.9,49.9,52.0,52.7,70.8,72.3,75.0,128.8$, 129.0, 129.1, 129.3, 129.5, 137.2. Found: $\mathrm{C}, 78.40 ; \mathrm{H}, 9.03 ; \mathrm{N}, 3.84$. Anal. Calcd for $\mathrm{C}_{24} \mathrm{H}_{33} \mathrm{NO}_{2}$ : C, 78.43; H, 9.05; N, 3.81.

(R)-2-((1R,2S,4R)-2-(Benzyloxy)-4-methylcyclohexyl)-1-(dibenzylamino)propan-2-ol (6a)

Prepared with 4 with dibenzylamine at reflux for $20 \mathrm{~h}$ and eluted by $n$-hexane:EtOAc $=9: 1$. Yield: 50\%, white crystal, m.p $=138-140{ }^{\circ} \mathrm{C} .[\alpha]_{\mathrm{D}}^{20}=+30.0(\mathrm{c} 0.27, \mathrm{MeOH}) .{ }^{1} \mathrm{H}$ NMR $(500 \mathrm{MHz}$, $\left.\mathrm{CDCl}_{3}\right): \delta=0.83-0.92(2 \mathrm{H}, \mathrm{m}), 0.84(3 \mathrm{H}, \mathrm{d}, J=6.5 \mathrm{~Hz}), 1.13(3 \mathrm{H}, \mathrm{s}), 1.53-1.56(4 \mathrm{H}, \mathrm{m}), 1.63-1.75$ $(3 \mathrm{H}, \mathrm{m}), 2.01(1 \mathrm{H}, \mathrm{dd}, J=14.1,1.9 \mathrm{~Hz}), 2.86(1 \mathrm{H}, \mathrm{d}, J=13.8 \mathrm{~Hz}), 3.3 .(1 \mathrm{H}, \mathrm{dd}, J=13.6,4.9 \mathrm{~Hz})$, $3.67(2 \mathrm{H}, \mathrm{d}, J=12.4 \mathrm{~Hz}), 4.06(1 \mathrm{H}, \mathrm{d}, J=11.9 \mathrm{~Hz}), 4.43(2 \mathrm{H}, \mathrm{t}, J=13.1 \mathrm{~Hz}), 4.53-4.62(3 \mathrm{H}, \mathrm{m})$, 7.13-7.57 $(15 \mathrm{H}, \mathrm{m}) .{ }^{13} \mathrm{C}$ NMR $\left(125 \mathrm{MHz} \mathrm{CDCl}_{3}\right): \delta=21.6,22.2,25.6,26.4,34.9,37.3,52.3$, 57.0, 59.5, 61.1, 69.5, 72.8, 74.3, 126.8, 127.5, 128.4, 129.2, 129.4, 130.1, 130.3, 132.2, 132.7, 139.1 . Found: C, 81.33; H, 8.63; N, 3.04. Anal. Calcd for $\mathrm{C}_{31} \mathrm{H}_{39} \mathrm{NO}_{2}$ : C, 81.36; $\mathrm{H}, 8.59 ; \mathrm{N}, 3.06$.

(S)-2-((1R,2S,4R)-2-(Benzyloxy)-4-methylcyclohexyl)-1-(dibenzylamino)propan-2-ol (6b)

Prepared with 4 with dibenzylamine at reflux for $20 \mathrm{~h}$ and eluted by $n$-hexane:EtOAc $=9: 1$. Yield: $25 \%$, white crystal, m.p $=164-166{ }^{\circ} \mathrm{C} .[\alpha]_{\mathrm{D}}^{20}=-4.0(\mathrm{c} 0.26, \mathrm{MeOH}) .{ }^{1} \mathrm{H}$ NMR $(500 \mathrm{MHz}$, $\left.\mathrm{CDCl}_{3}\right): \delta=0.82-0.89(1 \mathrm{H}, \mathrm{m}), 0.86(3 \mathrm{H}, \mathrm{d}, J=6.4 \mathrm{~Hz}), 1.29-1.33(2 \mathrm{H}, \mathrm{m}), 1.32(3 \mathrm{H}, \mathrm{s}), 1.56-1.61$ $(3 \mathrm{H}, \mathrm{m}), 1.73(1 \mathrm{H}, \mathrm{dd}, J=12.2,2.1 \mathrm{~Hz}), 2.10(1 \mathrm{H}, \mathrm{dd}, J=14.4,2.4 \mathrm{~Hz}), 2.63(1 \mathrm{H}, \mathrm{d}, J=13.2 \mathrm{~Hz})$, 3.48-3.53 (1H, m), 3.60-3.64 (1H, m), $4.04(1 \mathrm{H}, \mathrm{s}), 4.26(1 \mathrm{H}, \mathrm{d}, J=11.3 \mathrm{~Hz}), 4.47-4.66(5 \mathrm{H}, \mathrm{m})$, 7.25-7.65 (15H, m). $\left.{ }^{13} \mathrm{C} \mathrm{NMR} \mathrm{(125} \mathrm{MHz,} \mathrm{CDCl}_{3}\right): \delta=21.6,22.2,26.1,26.2,34.8,37.0,50.2$, $57.1,58.9,60.7,69.9,73.0,74.5,127.8,127.9,128.7,129.2,129.4,130.0,130.1,132.0,132.8,138.4$ Found: $\mathrm{C}, 81.40 ; \mathrm{H}, 8.55 ; \mathrm{N}, 3.07$. Anal. Calcd for $\mathrm{C}_{31} \mathrm{H}_{39} \mathrm{NO}_{2}$ : C, 81.36; $\mathrm{H}, 8.59 ; \mathrm{N}, 3.06$.

(S)-3-(Benzylamino)-2-((1R,2S,4R)-2-(Benzyloxy)-4-methylcyclohexyl)propane-1,2-diol (12a)

Prepared with 11a with benzylamine at reflux for $8 \mathrm{~h}$ and eluted by $n$-hexane:EtOAc $=1: 2$. Yield: $45 \%$, colorless oil. $[\alpha]_{\mathrm{D}}^{20}=+28.0(\mathrm{c} 0.40, \mathrm{MeOH}) .{ }^{1} \mathrm{H} \mathrm{NMR}\left(500 \mathrm{MHz}, \mathrm{CDCl}_{3}\right): \delta=0.82$ 
$0.89(1 \mathrm{H}, \mathrm{m}), 0.86(3 \mathrm{H}, \mathrm{d}, J=6.4 \mathrm{~Hz}), 1.29-1.33(2 \mathrm{H}, \mathrm{m}), 1.32(3 \mathrm{H}, \mathrm{s}), 1.56-1.61(3 \mathrm{H}, \mathrm{m}), 1.73$ $(1 \mathrm{H}, \mathrm{dd}, J=12.2,2.1 \mathrm{~Hz}), 2.10(1 \mathrm{H}, \mathrm{dd}, J=14.4,2.4 \mathrm{~Hz}), 2.63(1 \mathrm{H}, \mathrm{d}, J=13.2 \mathrm{~Hz}), 3.48-3.53(1 \mathrm{H}$, m), 3.60-3.64 (1H, m), $4.04(1 \mathrm{H}, \mathrm{s}), 4.26(1 \mathrm{H}, \mathrm{d}, J=11.3 \mathrm{~Hz}), 4.47-4.66(5 \mathrm{H}, \mathrm{m}), 7.25-7.65(15 \mathrm{H}$, m). ${ }^{13} \mathrm{C}$ NMR $\left(125 \mathrm{MHz}, \mathrm{CDCl}_{3}\right): \delta=21.6,22.2,26.1,26.2,34.8,37.0,50.2,57.1,58.9,60.7,69.9$, 73.0, 74.5, 127.8, 127.9, 128.7, 129.2, 129.4, 130.0, 130.1, 132.0, 132.8, 138.4. Found: C, 81.40; H, 8.55; N, 3.07. Anal. Calcd for $\mathrm{C}_{31} \mathrm{H}_{39} \mathrm{NO}_{2}$ : C, 81.36; $\mathrm{H}, 8.59 ; \mathrm{N}, 3.06$.

(R)-3-(Benzylamino)-2-((1R,2S,4R)-2-(Benzyloxy)-4-methylcyclohexyl)propane-1,2-diol (12b)

Prepared with 11a with benzylamine at reflux for $8 \mathrm{~h}$ and eluted by $n$-hexane:EtOAc $=1: 2$. Yield: $11 \%$, colorless oil. $[\alpha]_{\mathrm{D}}^{20}=+19.0$ (c $\left.0.30, \mathrm{MeOH}\right) .{ }^{1} \mathrm{H} \mathrm{NMR}\left(500 \mathrm{MHz}, \mathrm{CDCl}_{3}\right): \delta=0.86-$ $0.97(6 \mathrm{H}, \mathrm{m}), 0.88(3 \mathrm{H}, \mathrm{d}, J=6.4 \mathrm{~Hz}), 1.25-1.42(14 \mathrm{H}, \mathrm{m}), 1.57-1.61(3 \mathrm{H}, \mathrm{m}), 1.70-1.77(3 \mathrm{H}, \mathrm{m})$, 2.12-2.17 (1H, m), $2.76(2 \mathrm{H}, \mathrm{s}), 3.48(1 \mathrm{H}, \mathrm{s}), 3.62(1 \mathrm{H}, \mathrm{q}, J=11.2 \mathrm{~Hz}), 3.70(1 \mathrm{H}, \mathrm{q}, J=13.3 \mathrm{~Hz})$, $3.90(1 \mathrm{H}, \mathrm{s}), 4.23(1 \mathrm{H}, \mathrm{d}, J=11.0 \mathrm{~Hz}), 4.57(1 \mathrm{H}, \mathrm{d}, J=11.0 \mathrm{~Hz}), 7.22-7.38(10 \mathrm{H}, \mathrm{m}) .{ }^{13} \mathrm{C}$ NMR $\left(125 \mathrm{MHz}_{\mathrm{CDCl}}\right): \delta=21.1,22.3,26.1,34.9,37.1,45.1,54.0,54.9,67.3,70.0,74.4,74.8,127.7$, 128.2, 128.3, 128.6, 128.7, 128, 8, 137.8. Found: C, 81.33; H, 8.62; N, 3.11. Anal. Calcd for $\mathrm{C}_{31} \mathrm{H}_{39} \mathrm{NO}_{2}: \mathrm{C}, 81.36 ; \mathrm{H}, 8.59 ; \mathrm{N}, 3.06$.

(S)-2-((1R,2S,4R)-2-(Benzyloxy)-4-methylcyclohexyl)-3-(dibenzylamino)propane-1,2-diol (13a)

Prepared with 11a with dibenzylamine at reflux for $8 \mathrm{~h}$ and eluted by $n$-hexane:EtOAc $=4: 1$. Yield: $54 \%$, colorless oil. $[\alpha]_{\mathrm{D}}^{20}=-2.0(\mathrm{c} 0.26, \mathrm{MeOH}) .{ }^{1} \mathrm{H} \mathrm{NMR}\left(500 \mathrm{MHz}, \mathrm{CDCl}_{3}\right): \delta=0.87(3 \mathrm{H}$, $\mathrm{d}, J=6.2 \mathrm{~Hz}), 0.80-1.00(4 \mathrm{H}, \mathrm{m}), 1.10-1.25(1 \mathrm{H}, \mathrm{m}), 1.25-1.35(2 \mathrm{H}, \mathrm{m}), 1.45-1.80(7 \mathrm{H}, \mathrm{m}), 2.11$ $(1 \mathrm{H}, \mathrm{d}, J=14.0 \mathrm{~Hz}), 3.10(1 \mathrm{H}, \mathrm{d}, J=13.0 \mathrm{~Hz}), 3.32(1 \mathrm{H}, \mathrm{d}, J=8.6 \mathrm{~Hz}), 3.51(1 \mathrm{H}, \mathrm{d}, J=12.5 \mathrm{~Hz})$, $3.60(1 \mathrm{H}, \mathrm{brs}), 3.90-4.10(1 \mathrm{H}, \mathrm{m}), 4.04(1 \mathrm{H}, \mathrm{d}, J=12.6 \mathrm{~Hz}), 4.24(1 \mathrm{H}, \mathrm{s}), 4.30(1 \mathrm{H}, \mathrm{d}, J=11.1 \mathrm{H})$, $4.38(1 \mathrm{H}, \mathrm{d}, J=11.7 \mathrm{~Hz}), 4.95(1 \mathrm{H}, \mathrm{d}, J=11.9 \mathrm{~Hz}), 5.21(1 \mathrm{H}, \mathrm{s}), 5.91(1 \mathrm{H}, \mathrm{s}), 7.25-7.62(15 \mathrm{H}, \mathrm{m})$. ${ }^{13} \mathrm{C} \mathrm{NMR}\left(125 \mathrm{MHz} \mathrm{CDCl}_{3}\right): \delta=21.5,22.2,26.1,34.6,36.9,46.4,57.3,58.3,59.5,66.0,70.2,74.8$, 74.9, 128.1, 128.2, 128.7, 129.4, 129.6, 130.2, 130.4, 131.5, 138.3. Found: C, 78.63; H, 8.27; N, 3.00. Anal. Calcd for $\mathrm{C}_{31} \mathrm{H}_{39} \mathrm{NO}_{3}$ : C, 78.61; $\mathrm{H}, 8.30 ; \mathrm{N}, 2.96$.

(R)-2-((1R,2S,4R)-2-(Benzyloxy)-4-methylcyclohexyl)-3-(dibenzylamino)propane-1,2-diol (13b)

Prepared with $\mathbf{1 1 b}$ with dibenzylamine at reflux for $8 \mathrm{~h}$ and eluted by $n$-hexane:EtOAc $=4: 1$. Yield: $7 \%$, colorless oil. $[\alpha]_{\mathrm{D}}^{20}=+5.0(\mathrm{c} 0.20, \mathrm{MeOH}) .{ }^{1} \mathrm{H} \mathrm{NMR}\left(500 \mathrm{MHz}, \mathrm{CDCl}_{3}\right): \delta=0.84(3 \mathrm{H}$, $\mathrm{d}, J=6.5 \mathrm{~Hz}), 0.84-0.90(3 \mathrm{H}, \mathrm{m}), 1.26(3 \mathrm{H}, \mathrm{s}), 1.25-1.29(1 \mathrm{H}, \mathrm{m}), 1.48-1.55(1 \mathrm{H}, \mathrm{m}), 1.57-1.62$ $(1 \mathrm{H}, \mathrm{m}), 1.70-1.80(3 \mathrm{H}, \mathrm{m}), 2.05-2.13(1 \mathrm{H}, \mathrm{m}), 2.69(2 \mathrm{H}, \mathrm{m}), 3.37(1 \mathrm{H}, \mathrm{d}, J=11.3 \mathrm{~Hz}), 3.41(2 \mathrm{H}, \mathrm{d}$, $J=13.3 \mathrm{~Hz}), 3.51(1 \mathrm{H}, \mathrm{d}, J=11.3 \mathrm{~Hz}), 3.85(2 \mathrm{H}, \mathrm{d}, J=13.3 \mathrm{~Hz}), 4.00(1 \mathrm{H}, \mathrm{s}), 4.25(11.1 \mathrm{~Hz}), 4.55$ $(1 \mathrm{H}, \mathrm{d}, J=11.2 \mathrm{~Hz}), 7.23-7.49(15 \mathrm{H}, \mathrm{m}) .{ }^{13} \mathrm{C} \mathrm{NMR}\left(125 \mathrm{MHz}, \mathrm{CDCl}_{3}\right): \delta=20.6,22.3,26.2,34.9$, 37.0, 59.2, 60.6, 67.2, 69.9, 75.4, 75.6, 127.5, 127.9, 128.6, 128.7, 129.5, 139.0. Found: C, 78.57; H, 8.33; N, 2.94. Anal. Calcd for $\mathrm{C}_{31} \mathrm{H}_{39} \mathrm{NO}_{3}$ : C, 78.61; $\mathrm{H}, 8.30 ; \mathrm{N}, 2.96$.

(S)-1-(Benzylamino)-3-(Benzyloxy)-2-((1R,2S,4R)-2-(Benzyloxy)-4methylcyclohexyl)propan-2-ol (20a)

Prepared with 19a and benzylamine at reflux for $6 \mathrm{~h}$ and eluted by $n$-hexane:EtOAc $=2: 1$. Yield: $77 \%$, colorless oil. $[\alpha]_{\mathrm{D}}^{20}=+51.0$ (c $\left.0.25, \mathrm{MeOH}\right) .{ }^{1} \mathrm{H} \mathrm{NMR}\left(500 \mathrm{MHz}, \mathrm{CDCl}_{3}\right): \delta=0.87$ $(3 \mathrm{H}, \mathrm{d}, J=6.4 \mathrm{~Hz}), 0.86-0.95(2 \mathrm{H}, \mathrm{m}), 1.48-1.51(1 \mathrm{H}, \mathrm{m}), 1.64-1.76(4 \mathrm{H}, \mathrm{m}), 2.05-2.08(1 \mathrm{H}, \mathrm{m})$, $2.72(1 \mathrm{H}, \mathrm{dd}, J=16.4,11.7 \mathrm{~Hz}), 3.44(1 \mathrm{H}, \mathrm{d}, J=9.2 \mathrm{~Hz}), 3.50(1 \mathrm{H}, \mathrm{d}, J=9.2 \mathrm{~Hz}), 3.67(1 \mathrm{H}$, $\mathrm{d}, J=13.3 \mathrm{~Hz}), 3.78(1 \mathrm{H}, \mathrm{d}, J=13.4 \mathrm{~Hz}), 3.99(1 \mathrm{H}, \mathrm{s}), 4.13(1 \mathrm{H}, \mathrm{d}, J=11.2 \mathrm{~Hz}), 4.42(1 \mathrm{H}, \mathrm{d}$, $J=12.0 \mathrm{~Hz}), 4.49(1 \mathrm{H}, \mathrm{d}, J=11.2 \mathrm{~Hz}), 4.50(1 \mathrm{H}, \mathrm{d}, J=12.0 \mathrm{~Hz}), 7.22-7.32(15 \mathrm{H}, \mathrm{m}) .{ }^{13} \mathrm{C} \mathrm{NMR}$ $\left(125 \mathrm{MHz}_{\mathrm{CDCl}}\right): \delta=20.8,22.4,26.2,35.0,37.0,43.8,54.3,55.2,69.8,73.6,73.8,74.9,75.6$, 127.0, 127.7, 127.8, 128.0, 128.4, 128.5, 128.6, 138.4, 138.5. Found: C, 78.59; H, 8.33; N, 2.98. Anal. Calcd for $\mathrm{C}_{31} \mathrm{H}_{39} \mathrm{NO}_{3}$ : C, 78.61; H, 8.30; N, 2.96. 
(R)-1-(Benzylamino)-3-(Benzyloxy)-2-((1R,2S,4R)-2-(Benzyloxy)-4methylcyclohexyl)propan-2-ol (20b)

Prepared with $19 \mathrm{~b}$ and benzylamine at reflux for $6 \mathrm{~h}$ and eluted by $n$-hexane:EtOAc $=1: 2$. Yield: $84 \%$, colorless oil. $[\alpha]_{\mathrm{D}}^{20}=+42.0(\mathrm{c} 0.25, \mathrm{MeOH}) .{ }^{1} \mathrm{H} \mathrm{NMR}\left(500 \mathrm{MHz}, \mathrm{CDCl}_{3}\right): \delta=0.85-$ $0.97(3 \mathrm{H}, \mathrm{m}), 0.88(1 \mathrm{H}, \mathrm{d}, J=6.4 \mathrm{~Hz}), 1.25-1.29(1 \mathrm{H}, \mathrm{m}), 1.41-1.46(1 \mathrm{H}, \mathrm{m}), 1.57-1.65(2 \mathrm{H}, \mathrm{m})$, 1.71-1.77 (2H, m), 2.13-2.17 (1H, m), $2.74(1 \mathrm{H}, \mathrm{d}, J=12.2 \mathrm{~Hz}), 2.91(1 \mathrm{H}, \mathrm{d}, J=12.2 \mathrm{~Hz}), 3.58$ $(1 \mathrm{H}, \mathrm{d}, J=11.3 \mathrm{~Hz}), 3.62(1 \mathrm{H}, \mathrm{d}, J=11.3 \mathrm{~Hz}), 3.74(2 \mathrm{H}, \mathrm{s}), 4.10(1 \mathrm{H}, \mathrm{s}), 4.33(1 \mathrm{H}, \mathrm{d}, J=10.9 \mathrm{~Hz})$, $4.60(1 \mathrm{H}, \mathrm{d}, J=10.9 \mathrm{~Hz}), 7.20-7.35(10 \mathrm{H}, \mathrm{m}) .{ }^{13} \mathrm{C} \mathrm{NMR}\left(125 \mathrm{MHz}, \mathrm{CDCl}_{3}\right): \delta=21.2,22.3,26.1$, 34.9 , 37.0, 53.5, 53.8, 68.9, 70.2, 74.1, 75.2, 127.9, 128.2, 128.3, 128.8, 137.9. Found: C, 75.12; H, 8.70; N, 2.63. Anal. Calcd for $\mathrm{C}_{24} \mathrm{H}_{33} \mathrm{NO}_{3}$ : C, 75.16; $\mathrm{H}, 8.67 ; \mathrm{N}, 3.65$.

(S)-1-(Benzyloxy)-2-((1R,2S,4R)-2-(Benzyloxy)-4-methylcyclohexyl)-3(dibenzylamino)propan-2-ol (21a)

Prepared with 19a and dibenzylamine at reflux for $6 \mathrm{~h}$ and eluted by $n$-hexane:EtOAc $=9: 1$. Yield: $67 \%$, white crystal, m.p. $=54-55{ }^{\circ} \mathrm{C} .[\alpha]_{\mathrm{D}}^{20}=+39.0($ c $0.25, \mathrm{MeOH}) .{ }^{1} \mathrm{H} \mathrm{NMR}(500 \mathrm{MHz}$, $\left.\mathrm{CDCl}_{3}\right): \delta=0.83(3 \mathrm{H}, \mathrm{d}, J=6.5 \mathrm{~Hz}), 0.79-0.90(2 \mathrm{H}, \mathrm{m}), 1.39-1.41(1 \mathrm{H}, \mathrm{m}), 1.57-1.69(4 \mathrm{H}, \mathrm{m})$, 1.97-2.00 (1H, m), $2.70(1 \mathrm{H}, \mathrm{d}, J=13.9 \mathrm{~Hz}), 2.77(1 \mathrm{H}, \mathrm{d}, J=13.9 \mathrm{~Hz}), 3.33(1 \mathrm{H}, \mathrm{d}, J=9.1 \mathrm{~Hz}), 3.49$ $(1 \mathrm{H}, \mathrm{d}, J=13.7 \mathrm{~Hz}), 3.60(1 \mathrm{H}, \mathrm{d}, J=9.1 \mathrm{~Hz}), 3.77(1 \mathrm{H}, \mathrm{d}, J=13.7 \mathrm{~Hz}), 3.80(1 \mathrm{H}, \mathrm{d}, J=9.7 \mathrm{~Hz})$, $3.87(1 \mathrm{H}, \mathrm{s}), 4.12(1 \mathrm{H}, \mathrm{d}, J=11.5 \mathrm{~Hz}), 4.35(1 \mathrm{H}, \mathrm{d}, J=16.6 \mathrm{~Hz}), 4.66(1 \mathrm{H}, \mathrm{d}, J=16.7 \mathrm{~Hz}), 4.44$ $(1 \mathrm{H}, \mathrm{d}, J=11.4 \mathrm{~Hz}), 7.16-7.31(20 \mathrm{H}, \mathrm{m}) .{ }^{13} \mathrm{C} \mathrm{NMR}\left(125 \mathrm{MHz}, \mathrm{CDCl}_{3}\right): \delta=20.9,22.4,26.3,35.2$, 37.4, 45.4, 58.2, 60.0, 69.8, 73.1, 73.4, 75.3, 76.0, 126.9, 127.5, 127.6, 127.8, 127.9, 128.2, 128.3, $128.5,129.3,138.7,138.9,140.0$. Found: $\mathrm{C}, 80.95 ; \mathrm{H}, 8.03 ; \mathrm{N}, 2.50$. Anal. Calcd for $\mathrm{C}_{38} \mathrm{H}_{45} \mathrm{NO}_{3}$ : C, 80.96; H, 8.05; N, 2.48 .

(R)-1-(Benzyloxy)-2-((1R,2S,4R)-2-(Benzyloxy)-4-methylcyclohexyl)-3(dibenzylamino)propan-2-ol (21b)

Prepared with $\mathbf{1 9 b}$ and dibenzylamine at reflux for $6 \mathrm{~h}$ and eluted by $n$-hexane:EtOAc $=9: 1$. Yield: $53 \%$, colorless oil. $[\alpha]_{\mathrm{D}}^{20}=+26.0$ (c $\left.0.25, \mathrm{MeOH}\right) .{ }^{1} \mathrm{H} \mathrm{NMR}\left(500 \mathrm{MHz}, \mathrm{CDCl}_{3}\right): \delta=0.84$ $(3 \mathrm{H}, \mathrm{d}, J=6.5 \mathrm{~Hz}), 0.79-0.83(3 \mathrm{H}, \mathrm{m}), 1.51-1.58(4 \mathrm{H}, \mathrm{m}), 1.67-1.76(3 \mathrm{H}, \mathrm{m}), 1.97-2.01(1 \mathrm{H}$, m), $2.60(1 \mathrm{H}, \mathrm{d}, J=13.7 \mathrm{~Hz}), 2.71(1 \mathrm{H}, \mathrm{d}, J=13.6 \mathrm{~Hz}), 3.23(1 \mathrm{H}, \mathrm{d}, J=8.7 \mathrm{~Hz}), 3.40(2 \mathrm{H}, \mathrm{d}$, $J=13.9 \mathrm{~Hz}), 3.65(1 \mathrm{H}, \mathrm{s}), 3.73(1 \mathrm{H}, \mathrm{d}, J=8.7 \mathrm{~Hz}), 3.80(1 \mathrm{H}, \mathrm{s}), 3.88(2 \mathrm{H}, \mathrm{d}, J=13.9 \mathrm{~Hz}), 3.96$ $(1 \mathrm{H}, \mathrm{d}, J=12.7 \mathrm{~Hz}), 4.17(1 \mathrm{H}, \mathrm{d}, J=11.9 \mathrm{~Hz}), 4.34(1 \mathrm{H}, \mathrm{d}, J=12.0 \mathrm{~Hz}), 4.40(1 \mathrm{H}, \mathrm{d}, J=11.3 \mathrm{~Hz})$, 7.17-7.32 (20H, m). ${ }^{13} \mathrm{C} \mathrm{NMR}\left(125 \mathrm{MHz} \mathrm{CDCl}_{3}\right): \delta=20.8,22.4,26.2,35.0,36.9,44.3,57.5,59.8$, $69.5,72.5,73.2,75.9,76.5,126.7,127.6,127.7,127.8,128.1,128.2,128.3,128.5,129.2,138.5,138.6$, 140.2. Found: C, 81.00; H, 8.10; N, 2.45. Anal. Calcd for $\mathrm{C}_{38} \mathrm{H}_{45} \mathrm{NO}_{3}$ : C, 80.96; H, 8.05; N, 2.48 .

(1S,2R,5R)-2-((S)-1-(Benzylamino)-3-(Benzyloxy)-2-hydroxypropan-2-yl)-5methylcyclohexanol (25a)

Prepared with 25a and benzylamine at reflux for $8 \mathrm{~h}$ and eluted by $n$-hexane:EtOAc $=1: 2$. Yield: $71 \%$, colorless oil. $[\alpha]_{\mathrm{D}}^{20}=+18.0$ (c $\left.0.29, \mathrm{MeOH}\right) .{ }^{1} \mathrm{H} \mathrm{NMR}\left(500 \mathrm{MHz}, \mathrm{CDCl}_{3}\right): \delta=0.85$ $(3 \mathrm{H}, \mathrm{d}, J=6.4 \mathrm{~Hz}), 0.84-0.92(2 \mathrm{H}, \mathrm{m}), 1.00-1.06(1 \mathrm{H}, \mathrm{m}), 1.20-1.29(2 \mathrm{H}, \mathrm{m}), 1.53-1.62(2 \mathrm{H}, \mathrm{m})$, 1.70-1.73 (1H, m), 1.86-1.94 (2H, m), $2.64(1 \mathrm{H}, \mathrm{d}, J=11.9 \mathrm{~Hz}), 2.75(1 \mathrm{H}, \mathrm{d}, J=12.0 \mathrm{~Hz}), 3.33$ $(1 \mathrm{H}, \mathrm{d}, J=9.2 \mathrm{~Hz}), 3.38(1 \mathrm{H}, \mathrm{d}, J=9.2 \mathrm{~Hz}), 3.80(2 \mathrm{H}, \mathrm{s}), 4.23(1 \mathrm{H}, \mathrm{s}), 4.51(1 \mathrm{H}, \mathrm{d}, J=16.8 \mathrm{~Hz})$, $4.52(1 \mathrm{H}, \mathrm{d}, J=16.8 \mathrm{~Hz}), 7.25-7.35(10 \mathrm{H}, \mathrm{m}) .{ }^{13} \mathrm{C} \mathrm{NMR}\left(125 \mathrm{MHz}, \mathrm{CDCl}_{3}\right): \delta=21.5,22.4,26.0$, 35.5, 42.4, 48.2, 50.6, 53.8, 64.6, 73.8, 74.7, 75.1, 127.7, 127.9, 128.1, 128.6, 128.7, 128.8, 137.7, 138.1. Found: $\mathrm{C}, 75.13 ; \mathrm{H}, 8.65 ; \mathrm{N}, 3.70$. Anal. Calcd for $\mathrm{C}_{24} \mathrm{H}_{33} \mathrm{NO}_{3}: \mathrm{C}, 75.16 ; \mathrm{H}, 8.67 ; \mathrm{N}, 3.65$. 
(1S,2R,5R)-2-((R)-1-(Benzylamino)-3-(Benzyloxy)-2-hydroxypropan-2-yl)-5methylcyclohexanol (25b)

Prepared with $25 \mathrm{~b}$ and benzylamine at reflux for $8 \mathrm{~h}$ and eluted by $n$-hexane:EtOAc $=1: 2$. Yield: $85 \%$, colorless oil. $[\alpha]_{\mathrm{D}}^{20}=+4.0$ (c $\left.0.25, \mathrm{MeOH}\right) .{ }^{1} \mathrm{H}$ NMR $\left(500 \mathrm{MHz}, \mathrm{CDCl}_{3}\right): \delta=0.85$ $(3 \mathrm{H}, \mathrm{d}, J=6.4 \mathrm{~Hz}), 0.84-0.90(3 \mathrm{H}, \mathrm{m}), 0.98-1.03(1 \mathrm{H}, \mathrm{m}), 1.25-1.42(7 \mathrm{H}, \mathrm{m}), 1.48-1.56(2 \mathrm{H}$, m), 1.63-1.77 (2H, m), 1.82-1.90 (2H, m), $2.80(2 \mathrm{H}, \mathrm{s}), 3.37(1 \mathrm{H}, \mathrm{d}, J=9.3 \mathrm{~Hz}), 3.53(1 \mathrm{H}, \mathrm{d}$, $J=9.3 \mathrm{~Hz}), 3.77(2 \mathrm{H}, \mathrm{s}), 4.07(1 \mathrm{H}, \mathrm{s}), 4.47(1 \mathrm{H}, \mathrm{d}, J=11.9 \mathrm{~Hz}), 4.53(1 \mathrm{H}, \mathrm{d}, J=11.9 \mathrm{~Hz}), 7.24-7.34$ $(10 \mathrm{H}, \mathrm{m}) .{ }^{13} \mathrm{C} \mathrm{NMR}\left(125 \mathrm{MHz}, \mathrm{CDCl}_{3}\right): \delta=20.4,22.4,26.0,29.8,35.3,42.3,46.8,52.1,54.2,66.2$, 73.4, 73.6, 75.0, 127.6, 127.9, 128.1, 128.5, 128.7, 128.8, 137.8, 138.6. Found: C, 75.20; H, 8.70; N, 3.63. Anal. Calcd for $\mathrm{C}_{24} \mathrm{H}_{33} \mathrm{NO}_{3}$ : C, 75.16; $\mathrm{H}, 8.67 ; \mathrm{N}, 3.65$.

(1S,2R,5R)-2-((S)-1-(Benzyloxy)-3-(dibenzylamino)-2-hydroxypropan-2-yl)-5methylcyclohexanol (26a)

Prepared with 25a and dibenzylamine at reflux for $8 \mathrm{~h}$ and eluted by $n$-hexane:EtOAc $=9: 1$. Yield: $88 \%$, colorless oil. $[\alpha]_{\mathrm{D}}^{20}=+23.0(\mathrm{c} 0.25, \mathrm{MeOH}) .{ }^{1} \mathrm{H} \mathrm{NMR}\left(500 \mathrm{MHz}, \mathrm{CDCl}_{3}\right): \delta=0.82$ $(3 \mathrm{H}, \mathrm{d}, J=6.4 \mathrm{~Hz}), 0.76-0.89(2 \mathrm{H}, \mathrm{m}), 0.92-0.98(1 \mathrm{H}, \mathrm{m}), 1.19-1.45$ (3H, m), 1.60-1.64 (1H, m), $1.77-1.83(2 \mathrm{H}, \mathrm{m}), 2.67(1 \mathrm{H}, \mathrm{d}, J=14.0 \mathrm{~Hz}), 2.79(1 \mathrm{H}, \mathrm{d}, J=14.0 \mathrm{~Hz}), 3.25(1 \mathrm{H}, \mathrm{d}, J=9.0 \mathrm{~Hz})$, $3.42(1 \mathrm{H}, \mathrm{d}, J=9.0 \mathrm{~Hz}), 3.53(2 \mathrm{H}, \mathrm{d}, J=13.5 \mathrm{~Hz}), 3.80(2 \mathrm{H}, \mathrm{d}, J=13.5 \mathrm{~Hz}), 4.16(1 \mathrm{H}, \mathrm{s}), 4.33$ $(1 \mathrm{H}, \mathrm{d}, J=11.7 \mathrm{~Hz}), 4.39(1 \mathrm{H}, \mathrm{d}, J=11.7 \mathrm{~Hz}), 7.21-7.33(15 \mathrm{H}, \mathrm{m}) .{ }^{13} \mathrm{C} \mathrm{NMR}\left(125 \mathrm{MHz}, \mathrm{CDCl}_{3}\right)$ : $\delta=20.2,22.4,25.9,35.3,42.1,46.1,56.1,60.3,66.2,72.5,73.5,74.8,127.5,128.0,128.5,128.6$, 129.5, 137.6, 138.6. Found: C, 78.63; H, 8.33; N, 2.98. Anal. Calcd for $\mathrm{C}_{31} \mathrm{H}_{39} \mathrm{NO}_{3}$ : C, $78.61 ; \mathrm{H}$, 8.30; N, 2.96 .

(1S,2R,5R)-2-((R)-1-(Benzyloxy)-3-(dibenzylamino)-2-hydroxypropan-2-yl)-5methylcyclohexanol (26b)

Prepared with $\mathbf{2 5 b}$ and dibenzylamine at reflux for $8 \mathrm{~h}$ and eluted by $n$-hexane:EtOAc $=9: 1$. Yield: $88 \%$, colorless oil. $[\alpha]_{\mathrm{D}}^{20}=+8.0(\mathrm{c} 0.25, \mathrm{MeOH}) .{ }^{1} \mathrm{H} \mathrm{NMR}\left(500 \mathrm{MHz}, \mathrm{CDCl}_{3}\right): \delta=0.82$ $(3 \mathrm{H}, \mathrm{d}, J=6.3 \mathrm{~Hz}), 0.77-0.85(1 \mathrm{H}, \mathrm{m}), 0.88-0.94(1 \mathrm{H}, \mathrm{m}), 1.29-1.33(1 \mathrm{H}, \mathrm{m}), 1.39-1.42(1 \mathrm{H}, \mathrm{m})$, 1.49-1.55 (3H, m), 1.64-1.67 (1H, m), 1.77-1.80 (2H, m), $2.82(1 \mathrm{H}, \mathrm{d}, J=17.0 \mathrm{~Hz}), 2.83(1 \mathrm{H}$, d, $J=16.9 \mathrm{~Hz}), 3.16(1 \mathrm{H}, \mathrm{brs}), 3.18(1 \mathrm{H}, \mathrm{d}, J=9.1 \mathrm{~Hz}), 3.34(1 \mathrm{H}, \mathrm{d}, J=9.1 \mathrm{~Hz}), 3.61(2 \mathrm{H}, \mathrm{d}$, $J=13.5 \mathrm{~Hz}), 3.72(2 \mathrm{H}, \mathrm{d}, J=13.5 \mathrm{~Hz}), 3.76(1 \mathrm{H}, \mathrm{brs}), 4.06(1 \mathrm{H}, \mathrm{s}), 4.36(2 \mathrm{H}, \mathrm{s}), 7.22-7.33(15 \mathrm{H}$, m). ${ }^{13} \mathrm{C} \mathrm{NMR}\left(125 \mathrm{MHz}, \mathrm{CDCl}_{3}\right): \delta=20.6,22.4,25.8,35.4,42.2,44.6,57.0,60.6,66.7,71.7,73.5$, $75.4,127.5,128.0,128.5,128.7,129.4,137.8,139.1$. Found: C, 78.57; H, 8.35; N, 2.93. Anal. Calcd for $\mathrm{C}_{31} \mathrm{H}_{39} \mathrm{NO}_{3}$ : C, 78.61; $\mathrm{H}, 8.30 ; \mathrm{N}, 2.96$.

(S)-2-((1R,2R,4R)-2-(Benzyloxy)-4-methylcyclohexyl)-1-(dibenzylamino)propan-2-ol (30b)

Prepared with 29 and dibenzylamine at reflux for $20 \mathrm{~h}$ and eluted by $n$-hexane:EtOAc $=9: 1$. Yield: $47 \%$, colorless oil. $[\alpha]_{\mathrm{D}}^{20}=-40.0$ (c $\left.0.255, \mathrm{MeOH}\right) .{ }^{1} \mathrm{H} \mathrm{NMR}\left(500 \mathrm{MHz}, \mathrm{CDCl}_{3}\right): \delta=0.45-$ $0.53(1 \mathrm{H}, \mathrm{m}), 0.84-0.90(1 \mathrm{H}, \mathrm{m}), 0.93(3 \mathrm{H}, \mathrm{s}), 0.96(3 \mathrm{H}, \mathrm{d}, J=5.5 \mathrm{~Hz}), 1.04(1 \mathrm{H}, \mathrm{q}, J=12.0 \mathrm{~Hz})$, 1.23-1.43 (3H, m), $1.55(3 \mathrm{H}, \mathrm{s}), 2.03-2.08(1 \mathrm{H}, \mathrm{m}), 2.22-2.26(1 \mathrm{H}, \mathrm{m}), 2.31(1 \mathrm{H}, \mathrm{d}, J=13.6 \mathrm{~Hz})$, $2.45(1 \mathrm{H}, \mathrm{d}, J=13.7 \mathrm{~Hz}), 3.23(2 \mathrm{H}, \mathrm{d}, J=13.7 \mathrm{hz}), 3.54(1 \mathrm{H}, \mathrm{td}, J=10.6,3.9 \mathrm{~Hz}), 4.18(2 \mathrm{H}, \mathrm{d}$, $J=13.6 \mathrm{~Hz}), 4.39(1 \mathrm{H}, \mathrm{d}, J=11.0 \mathrm{~Hz}), 4.66(1 \mathrm{H}, \mathrm{d}, J=11.0 \mathrm{~Hz}), 5.25(1 \mathrm{H}, \mathrm{s}), 7.18-7.35(15 \mathrm{H}, \mathrm{m})$. ${ }^{13} \mathrm{C} \mathrm{NMR}\left(125 \mathrm{MHz}, \mathrm{CDCl}_{3}\right): \delta=22.3,23.6,26.6,31.8,35.8,40.0,47.9,59.8,61.4,70.2,76.8,81.2$, 126.7, 128.1, 128.3, 128.7, 129.5, 137.6, 140.4. Found: C, 81.37; H, 8.35; N, 2.93. Anal. Calcd for $\mathrm{C}_{31} \mathrm{H}_{39} \mathrm{NO}_{2}: \mathrm{C}, 81.36 ; \mathrm{H}, 8.33 ; \mathrm{N}, 2.94$.

(S)-2-((1R,2R,4R)-2-(Benzyloxy)-4-methylcyclohexyl)-3-(dibenzylamino)propane-1,2-diol (34a)

Prepared with 33 and dibenzylamine at reflux for $8 \mathrm{~h}$ and eluted by $n$-hexane:EtOAc $=9: 1$. Yield: $76 \%$, colorless oil. $[\alpha]_{\mathrm{D}}^{20}=-126.0$ (c $\left.0.30, \mathrm{MeOH}\right) .{ }^{1} \mathrm{H} \mathrm{NMR}\left(500 \mathrm{MHz}, \mathrm{CDCl}_{3}\right): \delta=0.55-$ $0.64(1 \mathrm{H}, \mathrm{m}), 0.77-0.86(1 \mathrm{H}, \mathrm{m}), 0.87-0.94(1 \mathrm{H}, \mathrm{m}), 0.90(3 \mathrm{H}, \mathrm{d}, J=6.5 \mathrm{~Hz}), 1.22-1.32(1 \mathrm{H}, \mathrm{m})$, 1.51-1.61 (5H, m), $2.22(1 \mathrm{H}, \mathrm{d}, J=12.1 \mathrm{~Hz}), 2.43(1 \mathrm{H}, \mathrm{d}, J=13.6 \mathrm{~Hz}), 3.13(2 \mathrm{H}, \mathrm{d}, J=13.3 \mathrm{~Hz})$, 
$3.38(1 \mathrm{H}, \mathrm{d}, J=11.5 \mathrm{~Hz}), 3.46(1 \mathrm{H} \mathrm{td}, J=10.5,3.95 \mathrm{~Hz}), 3.60(1 \mathrm{H}, \mathrm{d}, J=11.4 \mathrm{~Hz}), 4.14(2 \mathrm{H}, \mathrm{d}$, $J=13.3 \mathrm{~Hz}), 4.39(1 \mathrm{H}, \mathrm{d}, J=11.1 \mathrm{~Hz}), 4.68(1 \mathrm{H}, \mathrm{d}, J=11.1 \mathrm{~Hz}), 7.21-7.38(15 \mathrm{H}, \mathrm{m}) .{ }^{13} \mathrm{C}$ NMR $\left(125 \mathrm{MHz}, \mathrm{CDCl}_{3}\right): \delta=22.2,26.0,31.2,34.4,40.0,49.8,57.2,60.6,67.8,70.1,76.7,80.0,127.2$, $128.2,128.3,128.5,128.8,129.3,137.7,139.2$. Found: C, 78.58; H, 8.33; N, 2.94. Anal. Calcd for $\mathrm{C}_{31} \mathrm{H}_{39} \mathrm{NO}_{3}: \mathrm{C}, 78.61 ; \mathrm{H}, 8.30 ; \mathrm{N}, 2.96$.

(S)-1-(Benzyloxy)-2-((1R,2R,4R)-2-(Benzyloxy)-4-methylcyclohexyl)-3(dibenzylamino)propan-2-ol (38a)

Prepared with 37a and dibenzylamine at reflux for $6 \mathrm{~h}$ and eluted by $n$-hexane:EtOAc $=9: 1$. Yield: $80 \%$, colorless oil. $[\alpha]_{\mathrm{D}}^{20}=-68.0(\mathrm{c} 0.27, \mathrm{MeOH}) .{ }^{1} \mathrm{H} \mathrm{NMR}\left(500 \mathrm{MHz}, \mathrm{CDCl}_{3}\right): \delta=0.52$ $0.60(1 \mathrm{H}, \mathrm{m}), 0.79-0.87(1 \mathrm{H}, \mathrm{m}), 0.89(3 \mathrm{H}, \mathrm{d}, J=6.5 \mathrm{~Hz}), 0.89-0.96(1 \mathrm{H}, \mathrm{m}), 1.20-1.35(\mathrm{H}$, m), $1.45-1.55(3 \mathrm{H}, \mathrm{m}), 1.86(1 \mathrm{H}, \mathrm{td}, J=12.2,3.2 \mathrm{~Hz}), 2.16(1 \mathrm{H}, \mathrm{d}, J=12.2 \mathrm{~Hz}), 2.43(1 \mathrm{H}, \mathrm{d}$, $J=13.6 \mathrm{~Hz}), 2.61(1 \mathrm{H}, \mathrm{d}, J=13.6 \mathrm{~Hz}), 3.13(1 \mathrm{H}, \mathrm{d}, J=10.6 \mathrm{~Hz}), 3.19(2 \mathrm{H}, \mathrm{d}, J=13.6 \mathrm{~Hz})$, $3.45(1 \mathrm{H}, \mathrm{td}, J=10.6,3.9 \mathrm{~Hz}), 3.71(1 \mathrm{H}, \mathrm{d}, J=10.6 \mathrm{~Hz}), 4.04(2 \mathrm{H}, \mathrm{d}, J=13.6 \mathrm{~Hz}), 4.34(1 \mathrm{H}, \mathrm{d}$, $J=11.2 \mathrm{~Hz}), 4.42(1 \mathrm{H}, \mathrm{d}, J=12.1 \mathrm{~Hz}), 4.63(1 \mathrm{H}, \mathrm{d}, J=12.2 \mathrm{~Hz}), 4.64(1 \mathrm{H}, \mathrm{d}, J=11.2 \mathrm{~Hz}), 4.77$ (1H, brs), 7.16-7.34 (20H, m). ${ }^{13} \mathrm{C} \mathrm{NMR}\left(125 \mathrm{MHz}, \mathrm{CDCl}_{3}\right): \delta=22.2,26.5,31.5,34.6,40.2,48.6$, $57.7,60.2,70.0,73.8,74.3,77.7,80.1,126.8,127.4,127.9,128.1,128.2,128.3,128.6,129.2,138.1$, 139.0, 140.0. Found: $\mathrm{C}, 80.93 ; \mathrm{H}, 8.07 ; \mathrm{N}, 2.50$. Anal. Calcd for $\mathrm{C}_{38} \mathrm{H}_{45} \mathrm{NO}_{3}$ : C, 80.96; H, 8.05; $\mathrm{N}, 2.48$

(R)-1-(Benzyloxy)-2-((1R,2R,4R)-2-(Benzyloxy)-4-methylcyclohexyl)-3(dibenzylamino)propan-2-ol (38b)

Prepared with 37a and dibenzylamine at reflux for $6 \mathrm{~h}$ and eluted by $n$-hexane:EtOAc $=9: 1$. Yield: $67 \%$, colorless oil. $[\alpha]_{\mathrm{D}}^{20}=-37.0(\mathrm{c} 0.25, \mathrm{MeOH}) .{ }^{1} \mathrm{H} \mathrm{NMR}\left(500 \mathrm{MHz}, \mathrm{CDCl}_{3}\right): \delta=0.82$ $0.99(3 \mathrm{H}, \mathrm{m}), 0.93(3 \mathrm{H}, \mathrm{d}, J=6.5 \mathrm{~Hz}), 1.29-1.35(1 \mathrm{H}, \mathrm{m}), 1.44-1.50(1 \mathrm{H}, \mathrm{m}), 1.55(1 \mathrm{H}, \mathrm{brs})$, 2.00-2.04 (1H, m), $2.17(1 \mathrm{H}, \mathrm{dd}, J=12.1,1.4 \mathrm{~Hz}), 2.39(1 \mathrm{H}, \mathrm{d}, J=13.7 \mathrm{~Hz}), 2.62(1 \mathrm{H}, \mathrm{d}$, $J=13.7 \mathrm{~Hz}), 3.29(2 \mathrm{H}, \mathrm{s}), 3.33(2 \mathrm{H}, \mathrm{d}, J=13.7 \mathrm{~Hz}), 3.68(1 \mathrm{H}, \mathrm{td}, J=10.7,3.9 \mathrm{~Hz}), 4.08(2 \mathrm{H}, \mathrm{d}$, $J=13.7 \mathrm{~Hz}), 4.19(1 \mathrm{H}, \mathrm{d}, J=10.9 \mathrm{~Hz}), 4.41(2 \mathrm{H}, \mathrm{q}, J=12.1 \mathrm{~Hz}), 4.51(1 \mathrm{H}, \mathrm{d}, J=10.9 \mathrm{~Hz}), 5.35$ (1H, brs), 7.18-7.33 (20H, m). ${ }^{13} \mathrm{C} \mathrm{NMR}\left(125 \mathrm{MHz}, \mathrm{CDCl}_{3}\right): \delta=22.3,26.3,31.7,35.1,40.5,47.7$, 58.0, 59.9, 70.1, 73.7, 74.8, 77.9, 81.6, 126.7, 127.6, 127.9, 128.1, 128.4, 128.6, 129.5, 138.0, 138.7, 140.2. Found: $\mathrm{C}, 80.95 ; \mathrm{H}, 8.07 ; \mathrm{N}, 2.52$. Anal. Calcd for $\mathrm{C}_{38} \mathrm{H}_{45} \mathrm{NO}_{3}$ : C, 80.96; $\mathrm{H}, 8.05 ; \mathrm{N}, 2.48$.

(1R,2R,5R)-2-((S)-1-(Benzyloxy)-3-(dibenzylamino)-2-hydroxypropan-2-yl)-5methylcyclohexanol (41a)

Prepared with $37 \mathrm{~b}$ and dibenzylamine at reflux for $8 \mathrm{~h}$ and eluted by $n$-hexane:EtOAc $=9: 1$. Yield: $88 \%$, colorless oil. $[\alpha]_{\mathrm{D}}^{20}=-5.0(\mathrm{c} 0.285, \mathrm{MeOH}) .{ }^{1} \mathrm{H}$ NMR $\left(500 \mathrm{MHz}, \mathrm{CDCl}_{3}\right): \delta=0.60-0.69$ $(1 \mathrm{H}, \mathrm{m}), 0.74-0.83(1 \mathrm{H}, \mathrm{m}), 0.85-0.95(1 \mathrm{H}, \mathrm{m}), 0.86(3 \mathrm{H}, \mathrm{d}, J=6.5 \mathrm{~Hz}), 1.22-1.32(1 \mathrm{H}, \mathrm{m}), 1.45-1.55$ $(2 \mathrm{H}, \mathrm{m}), 1.55(1 \mathrm{H}, \mathrm{s}), 1.64-1.69(1 \mathrm{H}, \mathrm{m}), 1.86-1.89(1 \mathrm{H}, \mathrm{m}), 2.62(1 \mathrm{H}, \mathrm{d}, J=14.1 \mathrm{~Hz}), 2.83(1 \mathrm{H}$, $\mathrm{d}, J=14.1 \mathrm{~Hz}), 3.16(1 \mathrm{H}, \mathrm{d}, J=9.9 \mathrm{~Hz}), 3.41-3.47(2 \mathrm{H}, \mathrm{m}), 3.58(2 \mathrm{H}, \mathrm{d}, J=13.4 \mathrm{~Hz}), 3.75(2 \mathrm{H}$, $\mathrm{d}, J=13.4 \mathrm{~Hz}), 3.88(1 \mathrm{H}, \mathrm{brs}), 4.41(1 \mathrm{H}, \mathrm{d}, J=12.0 \mathrm{~Hz}), 4.52(1 \mathrm{H}, \mathrm{d}, J=12.0 \mathrm{~Hz}), 4.77(1 \mathrm{H}, \mathrm{brs})$, 7.24-7.32 (15H, m). ${ }^{13} \mathrm{C} \mathrm{NMR}\left(125 \mathrm{MHz}, \mathrm{CDCl}_{3}\right): \delta=22.1,25.6,31.2,34.7,44.4,49.3,54.9,60.4$, 71.0, 73.6, 74.8, 77.5, 127.4, 127.8, 128.0, 128.5, 128.6, 129.4, 138.2, 139.1. Found: C, 78.58; H, 8.27; $\mathrm{N}, 2.95$. Anal. Calcd for $\mathrm{C}_{31} \mathrm{H}_{39} \mathrm{NO}_{3}$ : C, 78.61; $\mathrm{H}, 8.30 ; \mathrm{N}, 2.96$.

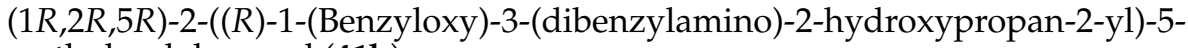
methylcyclohexanol (41b)

Prepared with $37 \mathrm{~b}$ and dibenzylamine at reflux for $8 \mathrm{~h}$ and eluted by $n$-hexane:EtOAc $=9: 1$. Yield: $76 \%$, colorless oil. $[\alpha]_{\mathrm{D}}^{20}=-22.0(\mathrm{c} 0.28, \mathrm{MeOH}) .{ }^{1} \mathrm{H} \mathrm{NMR}\left(500 \mathrm{MHz}, \mathrm{CDCl}_{3}\right): \delta=0.64$ $0.72(1 \mathrm{H}, \mathrm{m}), 0.87(3 \mathrm{H}, \mathrm{d}, J=6.4 \mathrm{~Hz}), 0.86-0.95(2 \mathrm{H}, \mathrm{m}), 1.26-1.49(5 \mathrm{H}, \mathrm{m}), 1.59(1 \mathrm{H}, \mathrm{brs}), 1.92$ $(1 \mathrm{H}, \mathrm{d}, J=12.4 \mathrm{~Hz}), 2.62(1 \mathrm{H}, \mathrm{d}, J=13.9 \mathrm{~Hz}), 2.87(1 \mathrm{H}, \mathrm{d}, J=13.9 \mathrm{~Hz}), 3.39(2 \mathrm{H}, \mathrm{s}), 3.51(1 \mathrm{H}, \mathrm{d}$, $J=13.4 \mathrm{~Hz}), 3.70(1 \mathrm{H}, \mathrm{td}, J=10.3,4.3 \mathrm{~Hz}), 3.87(1 \mathrm{H}, \mathrm{d}, J=13.4 \mathrm{~Hz}), 4.43(2 \mathrm{H}, \mathrm{t}, J=12.3 \mathrm{~Hz})$, 
7.24-7.32 (15H, m). $\left.{ }^{13} \mathrm{C} \mathrm{NMR} \mathrm{(125} \mathrm{MHz,} \mathrm{CDCl}_{3}\right): \delta=22.1,24.5,31.3,34.9,44.7,50.5,58.1,60.1$, 71.8, 73.2, 73.7, 76.3, 127.4, 127.8, 128.5, 128.6, 129.4, 138.1, 139.0. Found: C, 78.64; H, 8.33; N, 2.99. Anal. Calcd for $\mathrm{C}_{31} \mathrm{H}_{39} \mathrm{NO}_{3}$ : C, $78.61 ; \mathrm{H}, 8.30 ; \mathrm{N}, 2.96$.

4.2.6. General Procedure for Ring-Opening of Epoxide with Azoles

A solution of epoxides $(2.9 \mathrm{mmol})$ in dry DMF $(30 \mathrm{~mL})$ was added to the triazole or imidazole $(8.7 \mathrm{mmol})$ in dry DMF $(10 \mathrm{~mL})$ and $\mathrm{K}_{2} \mathrm{CO}_{3}(14.5 \mathrm{mmol})$. The mixture was kept at reflux temperature for 12-96 h. When the reaction completed (indicated by TLC), the mixture was dissolved in water $(15 \mathrm{~mL})$ and extracted with EtOAc $(3 \times 50 \mathrm{~mL})$. The combined organic phase was again extracted with saturated $\mathrm{NaCl}$ solution $(3 \times 50 \mathrm{~mL})$ then dried $\left(\mathrm{Na}_{2} \mathrm{SO}_{4}\right)$, filtered, and concentrated. The crude product was purified by column chromatography on silica gel with $\mathrm{CHCl}_{3}: \mathrm{MeOH}=19: 1$, resulting in O-benzyl derivatives, respectively.

(R)-2-((1R,2S,4R)-2-(Benzyloxy)-4-methylcyclohexyl)-1-(1H-imidazol-1-yl)propan-2-ol (7a)

Prepared with $4 \mathbf{a}$ and imidazole at reflux for $24 \mathrm{~h}$. Yield: $42 \%$, colorless oil. $[\alpha]_{\mathrm{D}}^{20}=+27.0$ (c $0.27, \mathrm{MeOH}) .{ }^{1} \mathrm{H} \mathrm{NMR}\left(500 \mathrm{MHz} \mathrm{CDCl}_{3}\right): \delta=0.86-0.97(3 \mathrm{H}, \mathrm{m}), 0.91(3 \mathrm{H}, \mathrm{d}, J=6.5 \mathrm{~Hz})$, $0.94(3 \mathrm{H}, \mathrm{s}), 1.23-1.42(7 \mathrm{H}, \mathrm{m}), 1.68-1.85(4 \mathrm{H}, \mathrm{m}), 2.22(1 \mathrm{H}, \mathrm{dd}, J=14.4,2.2 \mathrm{~Hz}), 3.82(2 \mathrm{H}, \mathrm{d}$, $J=2.8 \mathrm{~Hz}), 4.07(1 \mathrm{H}, \mathrm{s}), 4.33(1 \mathrm{H}, \mathrm{d}, J=11.4 \mathrm{~Hz}), 4.70(1 \mathrm{H}, \mathrm{d}, J=11.4 \mathrm{~Hz}), 6.85(1 \mathrm{H}, \mathrm{s}), 7.00$ $(1 \mathrm{H}, \mathrm{s}), 7.25-7.39(6 \mathrm{H}, \mathrm{m}) .{ }^{13} \mathrm{C}$ NMR $\left(125 \mathrm{MHz}, \mathrm{CDCl}_{3}\right): \delta=21.4,22.2,23.3,26.0,29.8,34.8$, $36.9,47.3,56.2,69.8,74.0,74.6,120.7,128.4,128.5,128.8,128.9,137.6,138.5$. Found: C, 73.10; $\mathrm{H}, 8.57 ; \mathrm{N}, 8.55$. Anal. Calcd for $\mathrm{C}_{20} \mathrm{H}_{28} \mathrm{~N}_{2} \mathrm{O}_{2}$ : C, 73.14; $\mathrm{H}, 8.59 ; \mathrm{N}, 8.53$.

(S)-2-((1R,2S,4R)-2-(Benzyloxy)-4-methylcyclohexyl)-1-(1H-imidazol-1-yl)propan-2-ol (7b)

Prepared with $\mathbf{4 b}$ and imidazole at reflux for $24 \mathrm{~h}$. Yield: $67 \%$, colorless oil. $[\alpha]_{\mathrm{D}}^{20}=+30.0$ (c $0.26, \mathrm{MeOH}) .{ }^{1} \mathrm{H}$ NMR $\left(500 \mathrm{MHz}, \mathrm{CDCl}_{3}\right): \delta=0.86-0.94(1 \mathrm{H}, \mathrm{m}), 0.91(3 \mathrm{H}, \mathrm{d}, J=6.6 \mathrm{~Hz})$, 0.96-1.02 (1H, m), $1.07(3 \mathrm{H}, \mathrm{s}), 1.25-1.31(2 \mathrm{H}, \mathrm{m}), 1.69-1.98(6 \mathrm{H}, \mathrm{m}), 2.16-2.22(1 \mathrm{H}, \mathrm{m})$, $3.82(1 \mathrm{H}, \mathrm{d}, J=14.1 \mathrm{~Hz}), 3.96(1 \mathrm{H}, \mathrm{d}, J=14.1 \mathrm{~Hz}), 4.06(1 \mathrm{H}, \mathrm{d}, J=1.7 \mathrm{~Hz}), 4.33(1 \mathrm{H}, \mathrm{d}$, $J=11.1 \mathrm{~Hz}), 4.65(1 \mathrm{H}, \mathrm{d}, J=11.1 \mathrm{~Hz}), 6.90(1 \mathrm{H}, \mathrm{s}), 7.02(1 \mathrm{H}, \mathrm{s}), 7.25-7.26(5 \mathrm{H}, \mathrm{m}), 7.43(1 \mathrm{H}$, s). ${ }^{13} \mathrm{C} \mathrm{NMR}\left(125 \mathrm{MHz} \mathrm{CDCl}_{3}\right): \delta=21.3,22.2,24.7,26.0,34.6,37.0,46.4,55.1,70.0,73.8$, 75.7, 120.7, 128.1, 128.2, 128.8, 129.0, 137.8, 138.5. Found: C, 73.17; H, 8.62; N, 8.50. Anal. Calcd for $\mathrm{C}_{20} \mathrm{H}_{28} \mathrm{~N}_{2} \mathrm{O}_{2}$ : C, 73.14; $\mathrm{H}, 8.59 ; \mathrm{N}, 8.53$.

(R)-2-((1R,2S,4R)-2-(Benzyloxy)-4-methylcyclohexyl)-1-(1H-1,2,4-triazol-1-yl)propan-2-ol (8a)

Prepared with 4a and 1,2,4-triazole at reflux for $24 \mathrm{~h}$. Yield: 67\%, colorless oil. $[\alpha]_{\mathrm{D}}^{20}=+42.0(\mathrm{c} 0.275, \mathrm{MeOH}) .{ }^{1} \mathrm{H}$ NMR $\left(500 \mathrm{MHz}, \mathrm{CDCl}_{3}\right): \delta=0.91(3 \mathrm{H}, \mathrm{d}, J=6.5 \mathrm{~Hz}), 0.94$ $(3 \mathrm{H}, \mathrm{s}), 0.90-0.98(2 \mathrm{H}, \mathrm{m}), 1.39-1.43(1 \mathrm{H}, \mathrm{m}), 1.61(1 \mathrm{H}, \mathrm{brs}), 1.71-1.84(4 \mathrm{H}, \mathrm{m}), 2.19-2.23(1 \mathrm{H}$, m), $4.00(1 \mathrm{H}, \mathrm{d}, J=13.9 \mathrm{~Hz}), 4.21(3 \mathrm{H}, \mathrm{t}, J=14.0 \mathrm{~Hz}), 4.34(1 \mathrm{H}, \mathrm{d}, J=11.3 \mathrm{~Hz}), 4.71(1 \mathrm{H}, \mathrm{d}$, $J=11.3 \mathrm{~Hz}), 7.26(1 \mathrm{H}, \mathrm{s}), 7.31-7.40(5 \mathrm{H}, \mathrm{m}), 7.89(1 \mathrm{H}, \mathrm{d}, J=2.8 \mathrm{~Hz}) .{ }^{13} \mathrm{C} \mathrm{NMR}(125 \mathrm{MHz}$, $\left.\mathrm{CDCl}_{3}\right): \delta=21.2,22.3,23.6,26.0,34.8,36.9,47.0,57.9,69.9,74.0,75.0,128.4,128.9,137.9$, 144.4, 151.6. Found: $\mathrm{C}, 69.30 ; \mathrm{H}, 8.24 ; \mathrm{N}, 12.80$. Anal. Calcd for $\mathrm{C}_{20} \mathrm{H}_{28} \mathrm{~N}_{2} \mathrm{O}_{2}: \mathrm{C}_{19} \mathrm{H}_{27} \mathrm{~N}_{3} \mathrm{O}_{2}$ : C, 69.27; H, 8.26; N, 12.76 .

(S)-2-((1R,2S,4R)-2-(Benzyloxy)-4-methylcyclohexyl)-1-(1H-1,2,4-triazol-1-yl)propan-2-ol (8b)

Prepared with $4 \mathrm{~b}$ and 1,2,4-triazole at reflux for $24 \mathrm{~h}$. Yield: 67\%, colorless oil. $[\alpha]_{\mathrm{D}}^{20}=+73.0(\mathrm{c} 0.28, \mathrm{MeOH}) .{ }^{1} \mathrm{H}$ NMR $\left(500 \mathrm{MHz}, \mathrm{CDCl}_{3}\right): \delta=0.86-1.03(2 \mathrm{H}, \mathrm{m}), 0.91$ $(3 \mathrm{H}, \mathrm{d}, J=6.5 \mathrm{~Hz}), 1.06(3 \mathrm{H}, \mathrm{s}), 1.37-1.41(1 \mathrm{H}, \mathrm{m}), 1.56(1 \mathrm{H}, \mathrm{s}), 1.71-1.90(4 \mathrm{H}, \mathrm{m}), 2.16-2.21$ $(1 \mathrm{H}, \mathrm{m}), 3.86(1 \mathrm{H}, \mathrm{s}), 4.10(1 \mathrm{H}, \mathrm{d}, J=1.8 \mathrm{~Hz}), 4.11(1 \mathrm{H}, \mathrm{d}, J=13.9 \mathrm{~Hz}), 4.21(1 \mathrm{H}, \mathrm{d}, J=14.0 \mathrm{~Hz})$, $4.34(1 \mathrm{H}, \mathrm{d}, J=11.2 \mathrm{~Hz}), 4.64(1 \mathrm{H}, \mathrm{d}, J=11.2 \mathrm{~Hz}), 7.25-7.35(5 \mathrm{H}, \mathrm{m}), 7.89(1 \mathrm{H}, \mathrm{s}), 8.06(1 \mathrm{H}$, s). ${ }^{13} \mathrm{C} \mathrm{NMR}\left(125 \mathrm{MHz}, \mathrm{CDCl}_{3}\right): \delta=21.4,22.3,24.7,26.0,47.0,57.2,70.0,73.7,75.4,128.1$, 128.7, 137.9, 144.6, 151.5. Found: C, 69.24; $\mathrm{H}, 8.30 ; \mathrm{N}, 12.73$. Anal. Calcd for $\mathrm{C}_{20} \mathrm{H}_{28} \mathrm{~N}_{2} \mathrm{O}_{2}$ : $\mathrm{C}_{19} \mathrm{H}_{27} \mathrm{~N}_{3} \mathrm{O}_{2}:$ C, 69.27; $\mathrm{H}, 8.26 ; \mathrm{N}, 12.76$. 
(S)-2-((1R,2S,4R)-2-(Benzyloxy)-4-methylcyclohexyl)-3-(1H-imidazol-1-yl)propane-1,2diol (14a)

Prepared with 11a and 1,2,4-triazole at reflux for $12 \mathrm{~h}$. Yield: 58\%, colorless oil. $[\alpha]_{\mathrm{D}}^{20}=+44.0(\mathrm{c} 0.25, \mathrm{MeOH}) .{ }^{1} \mathrm{H}$ NMR $\left(500 \mathrm{MHz}, \mathrm{CDCl}_{3}\right): \delta=0.91(3 \mathrm{H}, \mathrm{d}, J=6.6 \mathrm{~Hz})$, 0.94-1.03 (2H, m), 1.65-1.71 (2H, m), 1.77-1.80 (1H, m), 1.85-1.95 (2H, m), 2.10-2.20 (1H, m), $3.05(1 \mathrm{H}, \mathrm{d}, J=10.9 \mathrm{~Hz}), 3.32(1 \mathrm{H}, \mathrm{d}, J=10.9 \mathrm{~Hz}), 3.45(1 \mathrm{H}, \mathrm{s}), 4.00(1 \mathrm{H}, \mathrm{d}, J=14.0 \mathrm{~Hz}), 4.06$ $(1 \mathrm{H}, \mathrm{d}, J=14.0 \mathrm{~Hz}), 4.33(1 \mathrm{H}, \mathrm{d}, J=11.1 \mathrm{~Hz}), 4.63(1 \mathrm{H}, \mathrm{d}, J=11.1 \mathrm{~Hz}), 6.92(1 \mathrm{H}, \mathrm{s}), 6.96(1 \mathrm{H}$, s), 7.25-7.33 (5H, m), $7.46(1 \mathrm{H}, \mathrm{s}) .{ }^{13} \mathrm{C} \mathrm{NMR}\left(125 \mathrm{MHz}, \mathrm{CDCl}_{3}\right): \delta=20.8,22.3,26.0,34.7$, 36.8, 42.7, 50.7, 62.4, 69.9, 75.6, 75.8, 120.9, 128.1, 128.7, 137.7, 138.7. Found: C, 69.77; H, 8.16; $\mathrm{N}, 8.10$. Anal. Calcd for $\mathrm{C}_{20} \mathrm{H}_{28} \mathrm{~N}_{2} \mathrm{O}_{3}$ : C, 69.74; $\mathrm{H}, 8.19 ; \mathrm{N}, 8.13$.

(S)-2-((1R,2S,4R)-2-(Benzyloxy)-4-methylcyclohexyl)-3-(1H-1,2,4-triazol-1-yl)propane-1,2diol (15a)

Prepared with 11a and 1,2,4-triazole at reflux for $12 \mathrm{~h}$. Yield: 46\%, colorless oil. $[\alpha]_{\mathrm{D}}^{20}=+50.0(\mathrm{c} 0.26, \mathrm{MeOH}) .{ }^{1} \mathrm{H} \mathrm{NMR}\left(500 \mathrm{MHz}, \mathrm{CDCl}_{3}\right): \delta=0.86-0.93(1 \mathrm{H}, \mathrm{m}), 0.91(3 \mathrm{H}$, $\mathrm{d}, J=6.6 \mathrm{~Hz}), 0.95-1.05(2 \mathrm{H}, \mathrm{m}), 1.25-1.29(1 \mathrm{H}, \mathrm{m}), 1.55(2 \mathrm{H}, \mathrm{s}), 1.65-1.70(1 \mathrm{H}, \mathrm{m}), 1.73-1.85$ $(1 \mathrm{H}, \mathrm{m}), 1.84-1.93(2 \mathrm{H}, \mathrm{m}), 2.15-2.23(1 \mathrm{H}, \mathrm{m}), 2.99(1 \mathrm{H}, \mathrm{t}, J=7.6 \mathrm{~Hz}), 3.07(1 \mathrm{H}, \mathrm{dd}, J=12.0$, $4.4 \mathrm{~Hz}), 3.36(1 \mathrm{H}, \mathrm{dd}, J=11.9,7.9 \mathrm{~Hz}), 4.12(2 \mathrm{H}, \mathrm{s}), 4.28(1 \mathrm{H}, \mathrm{d}, J=6.9 \mathrm{~Hz}), 4.32(1 \mathrm{H}, \mathrm{d}$, $J=11.2 \mathrm{~Hz}), 4.64(1 \mathrm{H}, \mathrm{d}, J=11.1 \mathrm{~Hz}), 7.25-7.34(5 \mathrm{H}, \mathrm{m}), 7.92(1 \mathrm{H}, \mathrm{s}), 8.05(1 \mathrm{H}, \mathrm{s}) .{ }^{13} \mathrm{C}$ NMR $\left(125 \mathrm{MHz} \mathrm{CDCl}_{3}\right): \delta=20.8,22.2,26.0,34.6,36.7,42.5,53.7,64.3,69.9,75.2,75.9,128.2,128.8$, 137.5, 151.9. Found: C, 66.10; $\mathrm{H}, 7.83 ; \mathrm{N}, 12.11$. Anal. Calcd for $\mathrm{C}_{19} \mathrm{H}_{27} \mathrm{~N}_{3} \mathrm{O}_{3}$ : $\mathrm{C}, 66.06 ; \mathrm{H}$, $7.88 ; \mathrm{N}, 12.16$.

(S)-1-(Benzyloxy)-2-((1R,2S,4R)-2-(Benzyloxy)-4-methylcyclohexyl)-3-(1H-imidazol-1yl)propan-2-ol (22a)

Prepared with 19a and imidazole at reflux for $48 \mathrm{~h}$. Yield: $50 \%$, colorless oil. $[\alpha]_{\mathrm{D}}^{20}=+47.0$ (c $0.20, \mathrm{MeOH}) .{ }^{1} \mathrm{H}$ NMR $\left(500 \mathrm{MHz}, \mathrm{CDCl}_{3}\right): \delta=0.92(3 \mathrm{H}, \mathrm{d}, J=6.4 \mathrm{~Hz}), 0.91-0.97(2 \mathrm{H}, \mathrm{m})$, 1.52-1.81 (8H, m), $2.21(1 \mathrm{H}, \mathrm{dd}, J=14.3,2.4 \mathrm{~Hz}), 2.65(1 \mathrm{H}, \mathrm{d}, J=9.3 \mathrm{~Hz}), 3.17(1 \mathrm{H}, \mathrm{d}, J=9.3 \mathrm{~Hz})$, $3.81(1 \mathrm{H}, \mathrm{d}, J=13.8 \mathrm{~Hz}), 4.02(1 \mathrm{H}, \mathrm{d}, J=13.8 \mathrm{~Hz}), 4.02(1 \mathrm{H}, \mathrm{brs}), 4.11(1 \mathrm{H}, \mathrm{brs}), 4.27(1 \mathrm{H}, \mathrm{d}$, $J=11.7 \mathrm{~Hz}), 4.31(1 \mathrm{H}, \mathrm{d}, J=11.4 \mathrm{~Hz}), 4.40(1 \mathrm{H}, \mathrm{d}, J=11.7 \mathrm{~Hz}), 4.69(1 \mathrm{H}, \mathrm{d}, J=11.3 \mathrm{~Hz}), 6.84$ $(1 \mathrm{H}, \mathrm{s}), 6.98(1 \mathrm{H}, \mathrm{s}), 7.25-7.40(11 \mathrm{H}, \mathrm{m}) .{ }^{13} \mathrm{C} \mathrm{NMR}\left(125 \mathrm{MHz}, \mathrm{CDCl}_{3}\right): \delta=20.7,22.3,25.9,34.6$, 36.8, 42.7, 52.5, 69.1, 69.8, 73.2, 75.0, 75.4, 120.8, 127.9, 128.5, 128.6, 128.9, 137.4, 137.9, 138.6 Found: $\mathrm{C}, 74.63 ; \mathrm{H}, 7.93 ; \mathrm{N}, 6.47$. Anal. Calcd for $\mathrm{C}_{27} \mathrm{H}_{34} \mathrm{~N}_{2} \mathrm{O}_{3}$ : C, 74.62; $\mathrm{H}, 7.89 ; \mathrm{N}, 6.45$.

(R)-1-(Benzyloxy)-2-((1R,2S,4R)-2-(Benzyloxy)-4-methylcyclohexyl)-3-(1H-imidazol-1yl)propan-2-ol (22b)

Prepared with $19 \mathrm{~b}$ and imidazole at reflux for $48 \mathrm{~h}$. Yield: $42 \%$, colorless oil. $[\alpha]_{\mathrm{D}}^{20}=+71.0$ (c 0.20, MeOH). ${ }^{1} \mathrm{H}$ NMR $\left(500 \mathrm{MHz}, \mathrm{CDCl}_{3}\right): \delta=0.87-0.92(1 \mathrm{H}, \mathrm{m}), 0.95-1.03(1 \mathrm{H}, \mathrm{m}), 1.57-$ $1.68(6 \mathrm{H}, \mathrm{m}), 1.73-1.76(1 \mathrm{H}, \mathrm{m}), 1.82-1.87(1 \mathrm{H}, \mathrm{m}), 1.90-1.94(1 \mathrm{H}, \mathrm{m}), 2.09-1.13(1 \mathrm{H}, \mathrm{m}), 2.74$ $(1 \mathrm{H}, \mathrm{d}, J=9.4 \mathrm{~Hz}), 3.06(1 \mathrm{H}, \mathrm{d}, J=9.4 \mathrm{~Hz}), 3.82(1 \mathrm{H}, \mathrm{s}), 3.93(1 \mathrm{H}, \mathrm{d}, J=14.0 \mathrm{~Hz}), 3.95(1 \mathrm{H}$, $\mathrm{d}, J=14.1 \mathrm{~Hz}), 4.04(1 \mathrm{H}, \mathrm{d}, J=11.1 \mathrm{~Hz}), 4.08(1 \mathrm{H}, \mathrm{d}, J=13.9 \mathrm{~Hz}), 4.19(1 \mathrm{H}, \mathrm{d}, J=11.7 \mathrm{~Hz})$, $4.53(1 \mathrm{H}, \mathrm{d}, J=11.7 \mathrm{~Hz}), 4.50(1 \mathrm{H}, \mathrm{d}, J=11.1 \mathrm{~Hz}), 6.90(1 \mathrm{H}, \mathrm{s}), 6.99(1 \mathrm{H}, \mathrm{s}), 7.19-7.42(11 \mathrm{H}$, m). ${ }^{13} \mathrm{C} \mathrm{NMR}\left(125 \mathrm{MHz}, \mathrm{CDCl}_{3}\right): \delta=20.6,22.3,25.9,34.5,36.6,42.4,51.6,69.7,69.9,73.2,75.0$, $75.5,120.9,128.2,128.3,128.6,128.7,137.5$. Found: C, 74.59; H, 7.87; N, 6.43. Anal. Calcd for $\mathrm{C}_{27} \mathrm{H}_{34} \mathrm{~N}_{2} \mathrm{O}_{3}: \mathrm{C}, 74.62 ; \mathrm{H}, 7.89 ; \mathrm{N}, 6.45$.

(S)-1-(Benzyloxy)-2-((1R,2S,4R)-2-(Benzyloxy)-4-methylcyclohexyl)-3-(1H-1,2,4-triazol-1yl)propan-2-ol (23a)

Prepared with 19a and 1,2,4-triazole at reflux for $48 \mathrm{~h}$. Yield: 67\%, colorless oil. $[\alpha]_{\mathrm{D}}^{20}=+52.0(\mathrm{c} 0.25, \mathrm{MeOH}) .{ }^{1} \mathrm{H} \mathrm{NMR}\left(500 \mathrm{MHz}, \mathrm{CDCl}_{3}\right): \delta=0.89-0.98(2 \mathrm{H}, \mathrm{m}), 0.90(3 \mathrm{H}$, 
$\mathrm{d}, J=6.4 \mathrm{~Hz}), 1.54-1.65(3 \mathrm{H}, \mathrm{m}), 1.71-7.79(3 \mathrm{H}, \mathrm{m}), 2.18(1 \mathrm{H}, \mathrm{dd}, J=14.4,2.2 \mathrm{~Hz}), 3.05(1 \mathrm{H}$, $\mathrm{d}, J=9.7 \mathrm{~Hz}), 3.15(1 \mathrm{H}, \mathrm{d}, J=9.7 \mathrm{~Hz}), 4.22(2 \mathrm{H}, \mathrm{d}, J=14.1 \mathrm{~Hz}), 4.31-4.39(4 \mathrm{H}, \mathrm{m}), 4.46(1 \mathrm{H}$, $\mathrm{d}, J=11.7 \mathrm{~Hz}), 4.65(1 \mathrm{H}, \mathrm{d}, J=11.2 \mathrm{~Hz}), 7.25-7.36(10 \mathrm{H}, \mathrm{m}), 7.88(1 \mathrm{H}, \mathrm{s}), 7.94(1 \mathrm{H}, \mathrm{s}) .{ }^{13} \mathrm{C}$ NMR (125 MHz, $\left.\mathrm{CDCl}_{3}\right): \delta=20.8,22.3,26.0,34.7,36.8,43.4,53.7,69.9,71.3,73.6,75.1,75.6$, 128.0, 128.1, 128.2, 128.3, 128.5, 128.8, 137.8, 137.9, 144.8, 151.4. Found: C, 71.67; H, 7.69; N, 9.63. Anal. Calcd for $\mathrm{C}_{26} \mathrm{H}_{33} \mathrm{~N}_{3} \mathrm{O}_{3}$ : C, 71.70; $\mathrm{H}, 7.64 ; \mathrm{N}, 9.65$.

(R)-1-(Benzyloxy)-2-((1R,2S,4R)-2-(benzyloxy)-4-methylcyclohexyl)-3-(1H-1,2,4-triazol-1yl)propan-2-ol (23b)

Prepared with 19b and 1,2,4-triazole at reflux for $48 \mathrm{~h}$. Yield: 67\%, colorless oil. $[\alpha]_{\mathrm{D}}^{20}=+60.0(\mathrm{c} 0.25, \mathrm{MeOH}) .{ }^{1} \mathrm{H} \mathrm{NMR}\left(500 \mathrm{MHz}, \mathrm{CDCl}_{3}\right): \delta=0.88-0.94(2 \mathrm{H}, \mathrm{m}), 0.90(3 \mathrm{H}$, $\mathrm{d}, J=6.6 \mathrm{~Hz}), 0.98-1.02(1 \mathrm{H}, \mathrm{m}), 1.56(3 \mathrm{H}, \mathrm{s}), 1.63-1.89(5 \mathrm{H}, \mathrm{m}), 2.09-2.13(1 \mathrm{H}, \mathrm{m}), 2.94(1 \mathrm{H}$, $\mathrm{d}, J=9.6 \mathrm{~Hz}), 3.11(1 \mathrm{H}, \mathrm{d}, J=9.6 \mathrm{~Hz}), 3.90(1 \mathrm{H}, \mathrm{s}), 4.00(1 \mathrm{H}, \mathrm{s}), 4.09(1 \mathrm{H}, \mathrm{d}, J=11.1 \mathrm{~Hz})$, $4.27(1 \mathrm{H}, \mathrm{d}, J=11.7 \mathrm{~Hz}), 4.28(1 \mathrm{H}, \mathrm{d}, J=15.9 \mathrm{~Hz}), 4.35(1 \mathrm{H}, \mathrm{d}, J=14.0 \mathrm{~Hz}), 4.49(1 \mathrm{H}, \mathrm{d}$, $J=11.8 \mathrm{~Hz}), 4.52(1 \mathrm{H}, \mathrm{d}, J=11.1 \mathrm{~Hz}), 7.21-7.37(10 \mathrm{H}, \mathrm{m}), 7.89(1 \mathrm{H}, \mathrm{s}), 8.04(1 \mathrm{H}, \mathrm{s}) .{ }^{13} \mathrm{C}$ NMR $\left(125 \mathrm{MHz}_{2} \mathrm{CDCl}_{3}\right): \delta=20.7,22.3,26.0,34.6,36.7,42.6,53.4,69.8,70.8,73.4,74.9,75.3,128.0$, 128.1, 128.2, 128.3, 128.5, 128.7, 137.7, 144.9, 151.3. Found: C, 71.73; H, 7.60; N, 9.68. Anal. Calcd for $\mathrm{C}_{26} \mathrm{H}_{33} \mathrm{~N}_{3} \mathrm{O}_{3}$ : C, 71.70; $\mathrm{H}, 7.64 ; \mathrm{N}, 9.65$.

(1S,2R,5R)-2-((S)-1-(Benzyloxy)-2-hydroxy-3-(1H-imidazol-1-yl)propan-2-yl)-5methylcyclohexanol (27a)

Prepared with 25a and imidazole at reflux for $12 \mathrm{~h}$. Yield: 67\%, white crystal, m.p. $=118-119^{\circ} \mathrm{C} .[\alpha]_{\mathrm{D}}^{20}=+11.0($ c $0.30, \mathrm{MeOH}) .{ }^{1} \mathrm{H}$ NMR $\left(500 \mathrm{MHz}, \mathrm{CDCl}_{3}\right): \delta=0.89(3 \mathrm{H}$, $\mathrm{d}, J=6.2 \mathrm{~Hz}), 0.92-1.02(1 \mathrm{H}, \mathrm{m}), 1.12(1 \mathrm{H}, \mathrm{t}, J=12.2 \mathrm{~Hz}), 1.56-1.64(2 \mathrm{H}, \mathrm{m}), 1.74-1.90(4 \mathrm{H}$, m), $2.95(1 \mathrm{H}, \mathrm{d}, J=9.4 \mathrm{~Hz}), 3.40(1 \mathrm{H}, \mathrm{d}, J=9.4 \mathrm{~Hz}), 4.00(1 \mathrm{H}, \mathrm{d}, J=13.9 \mathrm{~Hz}), 4.11(1 \mathrm{H}, \mathrm{d}$, $J=13.9 \mathrm{~Hz}), 4.21(1 \mathrm{H}, \mathrm{s}), 4.37(1 \mathrm{H}, \mathrm{d}, J=11.7 \mathrm{~Hz}), 4.48(1 \mathrm{H}, \mathrm{d}, J=11.7 \mathrm{~Hz}), 6.90(1 \mathrm{H}, \mathrm{s}), 6.96$ $(1 \mathrm{H}, \mathrm{s}), 7.25-7.37(1 \mathrm{H}, \mathrm{s}), 7.43(1 \mathrm{H}, \mathrm{s}) .{ }^{13} \mathrm{C} \mathrm{NMR}\left(125 \mathrm{MHz}, \mathrm{CDCl}_{3}\right): \delta=20.0,22.2,25.8,34.7$, $42.5,42.9,51.4,68.1,70.3,73.5,75.5,120.8,128.1,128.2,128.6,128.7,137.7,138.7$. Found: C, 69.77; $\mathrm{H}, 8.15 ; \mathrm{N}, 8.12$. Anal. Calcd for $\mathrm{C}_{20} \mathrm{H}_{28} \mathrm{~N}_{2} \mathrm{O}_{3}$ : C, 69.74; $\mathrm{H}, 8.19 ; \mathrm{N}, 8.13$.

(1S,2R,5R)-2-((R)-1-(Benzyloxy)-2-hydroxy-3-(1H-imidazol-1-yl)propan-2-yl)-5methylcyclohexanol (27b)

Prepared with 25b and imidazole at reflux for $12 \mathrm{~h}$. Yield: 83\%, white crystal, m.p. $=149-150{ }^{\circ} \mathrm{C} .[\alpha]_{\mathrm{D}}^{20}=+20.0(\mathrm{c} 0.275, \mathrm{MeOH}) .{ }^{1} \mathrm{H} \mathrm{NMR}\left(500 \mathrm{MHz}, \mathrm{CDCl}_{3}\right): \delta=0.89(3 \mathrm{H}$, $\mathrm{d}, J=6.2 \mathrm{~Hz}), 0.90-0.95(1 \mathrm{H}, \mathrm{m}), 1.15(1 \mathrm{H}, \mathrm{td}, J=12.7,2.0 \mathrm{~Hz}), 1.46-1.50(1 \mathrm{H}, \mathrm{m}), 1.62-1.65$ $(1 \mathrm{H}, \mathrm{m}), 1.69-1.85(4 \mathrm{H}, \mathrm{m}), 2.81(1 \mathrm{H}, \mathrm{d}, J=9.3 \mathrm{~Hz}), 3.24(1 \mathrm{H}, \mathrm{d}, J=9.2 \mathrm{~Hz}), 4.17(2 \mathrm{H}, \mathrm{q}$, $J=14.0 \mathrm{~Hz}), 4.35(1 \mathrm{H}, \mathrm{d}, J=11.7 \mathrm{~Hz}), 4.37(1 \mathrm{H}, \mathrm{d}, J=1.5 \mathrm{~Hz}), 4.46(1 \mathrm{H}, \mathrm{d}, J=11.7 \mathrm{~Hz}), 6.94$ $(1 \mathrm{H}, \mathrm{s}), 6.97(1 \mathrm{H}, \mathrm{s}), 7.29-7.37(5 \mathrm{H}, \mathrm{m}), 747(1 \mathrm{H}, \mathrm{s}) .{ }^{13} \mathrm{C} \mathrm{NMR}\left(125 \mathrm{MHz} \mathrm{CDCl}_{3}\right): \delta=20.3$, 22.2, 25.8, 34.7, 42.6, 42.9, 52.4, 67.9, 69.3, 73.4, 75.8, 120.9, 128.0, 128.1, 128.5, 128.6, 137.7, 138.6. Found: $\mathrm{C}, 69.79 ; \mathrm{H}, 8.22 ; \mathrm{N}, 8.17$. Anal. Calcd for $\mathrm{C}_{20} \mathrm{H}_{28} \mathrm{~N}_{2} \mathrm{O}_{3}: \mathrm{C}, 69.74 ; \mathrm{H}, 8.19 ; \mathrm{N}$, 8.13 .

(1S,2R,5R)-2-((S)-1-(Benzyloxy)-2-hydroxy-3-(1H-1,2,4-triazol-1-yl)propan-2-yl)-5methylcyclohexanol (28a)

Prepared with 25a and 1,2,4-triazole at reflux for $12 \mathrm{~h}$. Yield: 83\%, colorless oil. $[\alpha]_{\mathrm{D}}^{20}=+12.0(\mathrm{c} 0.30, \mathrm{MeOH}) .{ }^{1} \mathrm{H} \mathrm{NMR}\left(500 \mathrm{MHz}, \mathrm{CDCl}_{3}\right): \delta=0.88(3 \mathrm{H}, \mathrm{d}, J=6.4 \mathrm{~Hz})$, 0.92-0.99 (1H, m), $1.07(1 \mathrm{H}, \mathrm{td}, J=12.0,1.7 \mathrm{~Hz}), 1.61-1.66(2 \mathrm{H}, \mathrm{m}), 1.78-1.91(4 \mathrm{H}, \mathrm{m}), 0.86$ $(1 \mathrm{H}, \mathrm{d}, J=9.5 \mathrm{~Hz}), 2.87(1 \mathrm{H}, \mathrm{s}), 2.94(1 \mathrm{H}, \mathrm{s}), 3.46(1 \mathrm{H}, \mathrm{d}, J=9.6 \mathrm{~Hz}), 4.22(1 \mathrm{H}, \mathrm{s}), 4.35-4.46$ $(4 \mathrm{H}, \mathrm{m}), 7.25-7.37(5 \mathrm{H}, \mathrm{m}), 7.92(1 \mathrm{H}, \mathrm{s}), 8.02(1 \mathrm{H}, \mathrm{s}) .{ }^{13} \mathrm{C} \mathrm{NMR}\left(125 \mathrm{MHz}, \mathrm{CDCl}_{3}\right): \delta=20.0$, $22.2,25.8,34.9,42.5,43.4,53.2,67.5,70.5,73.7,75.9,128.1,128.2,1287,137.5,151.9$. Found: C, 66.03; $\mathrm{H}, 7.90 ; \mathrm{N}, 12.20$. Anal. Calcd for $\mathrm{C}_{19} \mathrm{H}_{27} \mathrm{~N}_{3} \mathrm{O}_{3}: \mathrm{C}, 66.06 ; \mathrm{H}, 7.88 ; \mathrm{N}, 12.16$. 
(1S,2R,5R)-2-((R)-1-(Benzyloxy)-2-hydroxy-3-(1H-1,2,4-triazol-1-yl)propan-2-yl)-5methylcyclohexanol (28b)

Prepared with 25b and 1,2,4-triazole at reflux for $12 \mathrm{~h}$. Yield: 83\%, colorless oil. $[\alpha]_{\mathrm{D}}^{20}=+15.0(\mathrm{c} 0.25, \mathrm{MeOH}) .{ }^{1} \mathrm{H} \mathrm{NMR}\left(500 \mathrm{MHz}, \mathrm{CDCl}_{3}\right): \delta=0.88(3 \mathrm{H}, \mathrm{d}, J=6.3 \mathrm{~Hz})$, 0.87-0.95 (1H, m), $1.10(1 \mathrm{H}, \mathrm{td}, J=12.6,1.6 \mathrm{~Hz}), 1.22-1.29(1 \mathrm{H}, \mathrm{m}), 1.49-1.52(1 \mathrm{H}, \mathrm{m})$, 1.60-1.64 (1H, m), 1.70-1.81 (2H, m), 1.81-1.87 (2H, m), $2.81(1 \mathrm{H}, \mathrm{d}, J=9.6 \mathrm{~Hz}), 3.28(1 \mathrm{H}$, $\mathrm{d}, J=9.6 \mathrm{~Hz}), 4.35-4.52(5 \mathrm{H}, \mathrm{m}), 7.25-7.36(5 \mathrm{H}, \mathrm{m}), 7.93(1 \mathrm{H}, \mathrm{s}), 8.05(1 \mathrm{H}, \mathrm{s}) .{ }^{13} \mathrm{C} \mathrm{NMR}$ $\left(125 \mathrm{MHz}_{2} \mathrm{CDCl}_{3}\right): \delta=20.3,22.2,25.8,34.9,42.6,43.6,53.7,67.6,70.4,73.7,76.3,128.1,128.3$, 128.7, 137.5. Found: C, 66.10; $\mathrm{H}, 7.85 ; \mathrm{N}, 12.14$. Anal. Calcd for $\mathrm{C}_{19} \mathrm{H}_{27} \mathrm{~N}_{3} \mathrm{O}_{3}$ : $\mathrm{C}, 66.06 ; \mathrm{H}$, $7.88 ; \mathrm{N}, 12.16$.

(R)-2-((1R,2R,4R)-2-(Benzyloxy)-4-methylcyclohexyl)-1-(1H-imidazol-1-yl)propan-2-ol (31a)

Prepared with 29 and imidazole at reflux for 96 h. Yield: $38 \%$, colorless oil. $[\alpha]_{\mathrm{D}}^{20}=-34.0$ (c $0.20, \mathrm{MeOH}) .{ }^{1} \mathrm{H}$ NMR $\left(500 \mathrm{MHz}, \mathrm{CDCl}_{3}\right): \delta=0.80-1.01(2 \mathrm{H}, \mathrm{m}), 0.96(3 \mathrm{H}, \mathrm{d}, J=6.1 \mathrm{~Hz}), 1.01$ $(3 \mathrm{H}, \mathrm{s}), 1.10-1.20(1 \mathrm{H}, \mathrm{m}), 1.40-1.55(1 \mathrm{H}, \mathrm{m}), 1.72(1 \mathrm{H}, \mathrm{d}, J=12.7 \mathrm{~Hz}), 1.97(1 \mathrm{H}, \mathrm{d}, J=10.7 \mathrm{~Hz})$, $2.30(1 \mathrm{H}, \mathrm{d}, J=11.9 \mathrm{~Hz}), 3.46(1 \mathrm{H}, \mathrm{t}, J=7.8 \mathrm{~Hz}), 4.18(1 \mathrm{H}, \mathrm{s}), 4.35(1 \mathrm{H}, \mathrm{d}, J=10.9 \mathrm{~Hz}), 4.50(1 \mathrm{H}$, s), $4.76(1 \mathrm{H}, \mathrm{d}, J=10.8 \mathrm{~Hz}), 7.02(1 \mathrm{H}, \mathrm{s}), 7.16(1 \mathrm{H}, \mathrm{s}), 7.31-7.39(5 \mathrm{H}, \mathrm{m}), 9.26(1 \mathrm{H}, \mathrm{s}) .{ }^{13} \mathrm{C} \mathrm{NMR}$ $\left(125 \mathrm{MHz} \mathrm{CDCl}_{3}\right): \delta=22.1,26.0,31.6,34.5,40.1,49.7,57.6,70.6,73.4,80.3,118.2,122.9,128.6$, 128.7, 129.0, 137.7. Found: C, 73.17; $\mathrm{H}, 8.60 ; \mathrm{N}, 8.55$. Anal. Calcd for $\mathrm{C}_{20} \mathrm{H}_{28} \mathrm{~N}_{2} \mathrm{O}_{2}$ : C, 73.14; $\mathrm{H}$, $8.59 ; \mathrm{N}, 8.53$.

(S)-2-((1R,2R,4R)-2-(Benzyloxy)-4-methylcyclohexyl)-1-(1H-imidazol-1-yl)propan-2-ol (31b)

Prepared with 29 and imidazole at reflux for 96 h. Yield: 58\%, white crystal, m.p = 170 $172{ }^{\circ} \mathrm{C} .[\alpha]_{\mathrm{D}}^{20}=-48.0(\mathrm{c} 0.21, \mathrm{MeOH}) .{ }^{1} \mathrm{H} \mathrm{NMR}\left(500 \mathrm{MHz}, \mathrm{CDCl}_{3}\right): \delta=0.87-1.00(2 \mathrm{H}, \mathrm{m})$, $0.95(3 \mathrm{H}, \mathrm{d}, J=5.4 \mathrm{~Hz}), 1.05-1.16(4 \mathrm{H}, \mathrm{m}), 1.32(1 \mathrm{H}, \mathrm{s}), 1.43(1 \mathrm{H}, \mathrm{s}), 1.70-1.90(2 \mathrm{H}, \mathrm{m})$, $2.29(1 \mathrm{H}, \mathrm{d}, J=11.2 \mathrm{~Hz}), 3.62(1 \mathrm{H}, \mathrm{s}), 4.19(1 \mathrm{H}, \mathrm{brs}), 4.39(1 \mathrm{H}, \mathrm{d}, J=10.9 \mathrm{~Hz}), 4.70(1 \mathrm{H}, \mathrm{d}$, $J=10.9 \mathrm{~Hz}), 5.65(1 \mathrm{H}, \mathrm{s}), 7.28-7.38(7 \mathrm{H}, \mathrm{m}) .{ }^{13} \mathrm{C} \mathrm{NMR}\left(125 \mathrm{MHz}, \mathrm{CDCl}_{3}\right): \delta=22.0,27.5$, 31.3, 34.2, 39.5, 48.2, 70.4, 74.4, 80.2, 128.5, 128.6, 128.9. Found: C, 73.10; H, 8.55; N, 8.57. Anal. Calcd for $\mathrm{C}_{20} \mathrm{H}_{28} \mathrm{~N}_{2} \mathrm{O}_{2}: \mathrm{C}, 73.14 ; \mathrm{H}, 8.59 ; \mathrm{N}, 8.53$.

(R)-2-((1R,2R,4R)-2-(Benzyloxy)-4-methylcyclohexyl)-1-(1H-1,2,4-triazol-1-yl)propan-2-ol (32a)

Prepared with 29 and 1,2,4-triazole at reflux for $24 \mathrm{~h}$. Yield: 67\%, colorless oil. $[\alpha]_{\mathrm{D}}^{20}=-40.0$ (c 0.265, MeOH). ${ }^{1} \mathrm{H}$ NMR $\left(500 \mathrm{MHz}, \mathrm{CDCl}_{3}\right): \delta=0.85-0.94(1 \mathrm{H}, \mathrm{m}), 0.96$ $(3 \mathrm{H}, \mathrm{d}, J=6.6 \mathrm{~Hz}), 0.97(3 \mathrm{H}, \mathrm{s}), 1.05-1.13(1 \mathrm{H}, \mathrm{m}), 1.40-1.45(1 \mathrm{H}, \mathrm{m}), 1.50(1 \mathrm{H}, \mathrm{td}, J=9.7$, $3.1 \mathrm{~Hz}), 1.63(1 \mathrm{H}, \mathrm{brs}), 1.72(1 \mathrm{H}, \mathrm{d}, J=13.0 \mathrm{~Hz}), 1.93(1 \mathrm{H}, \mathrm{dd}, J=13.2,3.2 \mathrm{~Hz}), 2.28(1 \mathrm{H}, \mathrm{d}$, $J=12.1 \mathrm{~Hz}), 3.39(1 \mathrm{H}, \mathrm{td}, J=10.5,3.8 \mathrm{~Hz}), 4.12(2 \mathrm{H}, \mathrm{q}, J=13.8 \mathrm{~Hz}), 4.26(1 \mathrm{H}, \mathrm{d}, J=11.0 \mathrm{~Hz})$, $4.72(1 \mathrm{H}, \mathrm{d}, J=10.9 \mathrm{~Hz}), 4.98(1 \mathrm{H}, \mathrm{s}), 7.26-7.40(5 \mathrm{H}, \mathrm{m}), 7.88(1 \mathrm{H}, \mathrm{s}), 7.91(1 \mathrm{H}, \mathrm{s}) .{ }^{13} \mathrm{C}$ NMR $\left(125 \mathrm{MHz}_{2} \mathrm{CDCl}_{3}\right): \delta=22.1,23.4,26.5,31.5,34.6,39.9,50.8,57.1,70.3,74.4,80.3,128.5,128.6$, $129.0,137.5,144.8,151.1$. Found: $\mathrm{C}, 69.32 ; \mathrm{H}, 8.24 ; \mathrm{N}, 12.80$. Anal. Calcd for $\mathrm{C}_{19} \mathrm{H}_{27} \mathrm{~N}_{3} \mathrm{O}_{2}$ : C, 69.27; H, 8.26; N, 12.76 .

(S)-2-((1R,2R,4R)-2-(Benzyloxy)-4-methylcyclohexyl)-1-(1H-1,2,4-triazol-1-yl)propan-2-ol (32b)

Prepared with 29 and 1,2,4-triazole at reflux for $24 \mathrm{~h}$. Yield: 83\%, colorless oil. $[\alpha]_{\mathrm{D}}^{20}=-41.0(\mathrm{c} 0.285, \mathrm{MeOH}) .{ }^{1} \mathrm{H}$ NMR $\left(500 \mathrm{MHz}, \mathrm{CDCl}_{3}\right): \delta=0.78-0.87(1 \mathrm{H}, \mathrm{m}), 0.89-0.96$ $(1 \mathrm{H}, \mathrm{m}), 0.93(3 \mathrm{H}, \mathrm{d}, J=6.5 \mathrm{~Hz}), 0.97-1.04(1 \mathrm{H}, \mathrm{m}), 1.13(3 \mathrm{H}, \mathrm{s}), 1.15-1.21(1 \mathrm{H}, \mathrm{m}), 1.35-1.45$ $(1 \mathrm{H}, \mathrm{m}), 1.62(1 \mathrm{H}, \mathrm{s}), 1.66(1 \mathrm{H}, \mathrm{d}, J=13.1 \mathrm{~Hz}), 2.09(1 \mathrm{H}, \mathrm{dd}, J=12.8,3.1 \mathrm{~Hz}), 2.23(1 \mathrm{H}, \mathrm{d}$, $J=12.1 \mathrm{~Hz}), 3.59(1 \mathrm{H}, \mathrm{td}, J=10.4,3.8 \mathrm{~Hz}), 3.99(1 \mathrm{H}, \mathrm{d}, J=14.2 \mathrm{~Hz}), 4.26(1 \mathrm{H}, \mathrm{d}, J=14.1 \mathrm{~Hz})$, $4.39(1 \mathrm{H}, \mathrm{d}, J=11.0 \mathrm{~Hz}), 4.67(1 \mathrm{H}, \mathrm{d}, J=11.0 \mathrm{~Hz}), 5.46(1 \mathrm{H}, \mathrm{s}), 7.29-7.35(5 \mathrm{H}, \mathrm{m}), 7.87(1 \mathrm{H}$, s), $8.31(1 \mathrm{H}, \mathrm{s}) .{ }^{13} \mathrm{C} \mathrm{NMR}\left(125 \mathrm{MHz}, \mathrm{CDCl}_{3}\right): \delta=21.5,22.1,27.0,31.4,34.0,39.5,47.4,58.0$, 
70.2, 74.8, 80.4, 128.3, 128.4, 128.8, 137.2, 145.2, 150.7. Found: C, 69.25; H, 8.28; N, 12.73. Anal. Calcd for $\mathrm{C}_{19} \mathrm{H}_{27} \mathrm{~N}_{3} \mathrm{O}_{2}$ : C, 69.27; $\mathrm{H}, 8.26 ; \mathrm{N}, 12.76$.

(S)-2-((1R,2R,4R)-2-(Benzyloxy)-4-methylcyclohexyl)-3-(1H-imidazol-1-yl)propane-1,2diol (35a)

Prepared with 33 and imidazole at reflux for 12 h. Yield: 67\%, white crystal, m.p. = 135$136{ }^{\circ} \mathrm{C} .[\alpha]_{\mathrm{D}}^{20}=-42.0$ (c $\left.0.25, \mathrm{MeOH}\right) .{ }^{1} \mathrm{H}$ NMR $\left(500 \mathrm{MHz}, \mathrm{CDCl}_{3}\right): \delta=0.92-1.02(2 \mathrm{H}, \mathrm{m})$, $0.96(3 \mathrm{H}, \mathrm{d}, J=6.5 \mathrm{~Hz}), 1.03-1.09(1 \mathrm{H}, \mathrm{m}), 1.35-1.43(1 \mathrm{H}, \mathrm{m}), 1.69-1.72(1 \mathrm{H}, \mathrm{m}), 1.83-1.92$ $(3 \mathrm{H}, \mathrm{m}), 2.27(1 \mathrm{H}, \mathrm{d}, J=10.9 \mathrm{~Hz}), 3.21(1 \mathrm{H}, \mathrm{d}, J=11.3 \mathrm{~Hz}), 3.32(1 \mathrm{H}, \mathrm{td}, J=10.6,4.0 \mathrm{~Hz}), 3.39$ $(1 \mathrm{H}, \mathrm{d}, J=11.2 \mathrm{~Hz}), 3.90(2 \mathrm{H}, \mathrm{s}), 4.21(1 \mathrm{H}, \mathrm{d}, J=11.0 \mathrm{~Hz}), 4.64(1 \mathrm{H}, \mathrm{d}, J=11.0 \mathrm{~Hz}), 6.93(1 \mathrm{H}$, s), $7.02(1 \mathrm{H}, \mathrm{s}), 7.30-7.39(5 \mathrm{H}, \mathrm{m}), 7.44(1 \mathrm{H}, \mathrm{s}) .{ }^{13} \mathrm{C} \mathrm{NMR}\left(125 \mathrm{MHz}, \mathrm{CDCl}_{3}\right): \delta=22.1,26.5$, 31.3, 34.4, 40.1, 46.7, 50.5, 65.8, 70.0, 76.3, 79.7, 121.0, 128.5, 128.6, 128.9, 129.0, 137.2, 138.5 . Found: $\mathrm{C}, 69.77 ; \mathrm{H}, 8.17 ; \mathrm{N}, 8.10$. Anal. Calcd for $\mathrm{C}_{20} \mathrm{H}_{28} \mathrm{~N}_{2} \mathrm{O}_{3}: \mathrm{C}, 69.74 ; \mathrm{H}, 8.19 ; \mathrm{N}, 8.13$.

(R)-2-((1R,2R,4R)-2-(Benzyloxy)-4-methylcyclohexyl)-3-(1H-imidazol-1-yl)propane-1,2diol (35b)

Prepared with 33 and imidazole at reflux for $12 \mathrm{~h}$. Yield: $50 \%$, colorless oil. $[\alpha]_{\mathrm{D}}^{20}=-45.0$ (c $0.185, \mathrm{MeOH}) .{ }^{1} \mathrm{H}$ NMR $\left(500 \mathrm{MHz}, \mathrm{CDCl}_{3}\right): \delta=0.78-0.87(1 \mathrm{H}, \mathrm{m}), 0.88-0.96(1 \mathrm{H}, \mathrm{m}), 0.92$ $(3 \mathrm{H}, \mathrm{d}, J=6.5 \mathrm{~Hz}), 1.13-1.19(1 \mathrm{H}, \mathrm{m}), 1.35-1.41(2 \mathrm{H}, \mathrm{m}), 1.67(1 \mathrm{H}, \mathrm{d}, J=13.2 \mathrm{~Hz}), 1.75(1 \mathrm{H}$, $\mathrm{dd}, J=13.1,2.9 \mathrm{~Hz}), 1.85-2.10(2 \mathrm{H}, \mathrm{m}), 2.22(1 \mathrm{H}, \mathrm{d}, J=12.3 \mathrm{~Hz}), 3.40(1 \mathrm{H}, \mathrm{d}, J=11.2 \mathrm{~Hz})$, $3.50(1 \mathrm{H}, \mathrm{t}, J=11.1 \mathrm{~Hz}), 3.70(1 \mathrm{H}, \mathrm{td}, J=10.5,3.8 \mathrm{~Hz}), 3.90(1 \mathrm{H}, \mathrm{d}, J=14.4 \mathrm{~Hz}), 4.06(1 \mathrm{H}, \mathrm{d}$, $J=14.5 \mathrm{~Hz}), 4.37(1 \mathrm{H}, \mathrm{d}, J=11.1 \mathrm{~Hz}), 4.66(1 \mathrm{H}, \mathrm{d}, J=11.1 \mathrm{~Hz}), 7.01(2 \mathrm{H}, \mathrm{s}), 7.25-7.37(5 \mathrm{H}$, m), $7.54(1 \mathrm{H}, \mathrm{s}) .{ }^{13} \mathrm{C} \mathrm{NMR}\left(125 \mathrm{MHz} \mathrm{CDCl}_{3}\right): \delta=22.0,26.7,31.4,34.2,39.9,45.6,51.6,64.7$, 70.2, 76.5, 80.0, 121.0, 128.4, 128.5, 128.6, 128.9, 137.1, 138.7. Found: C, 69.73; H, 8.22; N, 8.17. Anal. Calcd for $\mathrm{C}_{20} \mathrm{H}_{28} \mathrm{~N}_{2} \mathrm{O}_{3}$ : C, 69.74; $\mathrm{H}, 8.19 ; \mathrm{N}, 8.13$.

(S)-2-((1R,2R,4R)-2-(Benzyloxy)-4-methylcyclohexyl)-3-(1H-1,2,4-triazol-1-yl)propane-1,2diol (36a)

Prepared with 33 and 1,2,4-triazole at reflux for $12 \mathrm{~h}$. Yield: 58\%, colorless oil. $[\alpha]_{\mathrm{D}}^{20}=-32.0$ (c 0.26, MeOH). ${ }^{1} \mathrm{H}$ NMR (500 MHz, $\left.\mathrm{CDCl}_{3}\right): \delta=0.88-1.01(2 \mathrm{H}, \mathrm{m}), 0.97$ $(3 \mathrm{H}, \mathrm{d}, J=6.5 \mathrm{~Hz}), 1.07-1.15(1 \mathrm{H}, \mathrm{m}), 1.39-1.46(1 \mathrm{H}, \mathrm{m}), 1.70-1.80(2 \mathrm{H}, \mathrm{m}), 1.95-2.00(1 \mathrm{H}$, $\mathrm{m}), 2.30(1 \mathrm{H}, \mathrm{dd}, J=12.3,1.5 \mathrm{~Hz}), 3.28(2 \mathrm{H}, \mathrm{dd}, J=13.5,12.2 \mathrm{~Hz}), 3.46(1 \mathrm{H}, \mathrm{td}, J=10.5$, $4.0 \mathrm{~Hz}), 4.03(1 \mathrm{H}, \mathrm{d}, J=14.1 \mathrm{~Hz}), 4.27(1 \mathrm{H}, \mathrm{d}, J=11.1 \mathrm{~Hz}), 4.28(1 \mathrm{H}, \mathrm{d}, J=14.1 \mathrm{~Hz}), 4.70(1 \mathrm{H}$, $\mathrm{d}, J=11.1 \mathrm{~Hz}), 7.33-7.42(5 \mathrm{H}, \mathrm{m}), 7.91(1 \mathrm{H}, \mathrm{s}), 7.92(1 \mathrm{H}, \mathrm{s}) .{ }^{13} \mathrm{C} \mathrm{NMR}\left(125 \mathrm{MHz}, \mathrm{CDCl}_{3}\right)$ : $\delta=22.1,26.1,31.2,34.5,40.0,48.2,52.9,66.1,70.0,76.1,79.5,128.7,129.0,137.2,151.2$. Found: C, 66.10; $\mathrm{H}, 7.89 ; \mathrm{N}, 12.12$. Anal. Calcd for $\mathrm{C}_{19} \mathrm{H}_{27} \mathrm{~N}_{3} \mathrm{O}_{3}: \mathrm{C}, 66.06 ; \mathrm{H}, 7.88 ; \mathrm{N}, 12.16$.

(R)-2-((1R,2R,4R)-2-(Benzyloxy)-4-methylcyclohexyl)-3-(1H-1,2,4-triazol-1-yl)propane1,2-diol (36b)

Prepared with 33 and 1,2,4-triazole at reflux for $12 \mathrm{~h}$. Yield: 50\%, colorless oil. $[\alpha]_{\mathrm{D}}^{20}=-32.0(\mathrm{c} 0.24, \mathrm{MeOH}) .{ }^{1} \mathrm{H}$ NMR $\left(500 \mathrm{MHz}, \mathrm{CDCl}_{3}\right): \delta=0.79-0.97(3 \mathrm{H}, \mathrm{m}), 0.93(3 \mathrm{H}$, $\mathrm{d}, J=6.5 \mathrm{~Hz}), 1.07-1.17(1 \mathrm{H}, \mathrm{m}), 1.26(2 \mathrm{H}, \mathrm{s}), 1.28-1.43(4 \mathrm{H}, \mathrm{m}), 1.69(1 \mathrm{H}, \mathrm{d}, J=13.2 \mathrm{~Hz})$, 2.06-2.09 (1H, m), $2.23(1 \mathrm{H}, \mathrm{d}, J=12.2 \mathrm{~Hz}), 3.43-3.49(2 \mathrm{H}, \mathrm{m}), 3.68(1 \mathrm{H}, \mathrm{td}, J=10.5,3.9 \mathrm{~Hz})$, $4.23(1 \mathrm{H}, \mathrm{d}, J=14.4 \mathrm{~Hz}), 4.35(1 \mathrm{H}, \mathrm{d}, J=14.3 \mathrm{~Hz}), 4.36(1 \mathrm{H}, \mathrm{d}, J=11.0 \mathrm{~Hz}), 4.66(1 \mathrm{H}, \mathrm{d}$, $J=11.1 \mathrm{~Hz}), 5.50$ (1H, brs), 7.25-7.37 (5H, m), $7.91(1 \mathrm{H}, \mathrm{s}), 8.23(1 \mathrm{H}, \mathrm{s}) .{ }^{13} \mathrm{C} \mathrm{NMR}(125 \mathrm{MHz}$, $\left.\mathrm{CDCl}_{3}\right): \delta=22.0,26.4,29.8,31.3,34.3,39.9,46.2,54.3,64.4,70.2,76.4,80.0,128.4,128.5,128.9$, 137.1, 150.6. Found: C, 66.03; H, 7.92; N, 12.18. Anal. Calcd for $\mathrm{C}_{19} \mathrm{H}_{27} \mathrm{~N}_{3} \mathrm{O}_{3}$ : C, 66.06; $\mathrm{H}$, 7.88; N, 12.16 . 
(S)-1-(Benzyloxy)-2-((1R,2R,4R)-2-(benzyloxy)-4-methylcyclohexyl)-3-(1H-imidazol-1yl)propan-2-ol (39a)

Prepared with 35a and imidazole at reflux for $48 \mathrm{~h}$. Yield: $67 \%$, colorless oil. $[\alpha]_{\mathrm{D}}^{20}=-72.0$ (c $0.28, \mathrm{MeOH}) .{ }^{1} \mathrm{H}$ NMR $\left(500 \mathrm{MHz}, \mathrm{CDCl}_{3}\right): \delta=0.86-1.10(3 \mathrm{H}, \mathrm{m}), 0.96(3 \mathrm{H}, \mathrm{d}, J=6.5 \mathrm{~Hz})$, 1.37-1.42 (1H, m), $1.69(1 \mathrm{H}, \mathrm{d}, J=12.6 \mathrm{~Hz}), 1.79(1 \mathrm{H}, \mathrm{td}, J=12.6,3.2 \mathrm{~Hz}), 2.00-2.04(2 \mathrm{H}, \mathrm{m})$, $2.28(1 \mathrm{H}, \mathrm{d}, J=12.1 \mathrm{~Hz}), 3.13(2 \mathrm{H}, \mathrm{d}, J=8.9 \mathrm{~Hz}), 3.39(1 \mathrm{H}, \mathrm{td}, J=10.6,3.9 \mathrm{~Hz}), 3.83(1 \mathrm{H}, \mathrm{d}$, $J=13.9 \mathrm{~Hz}), 4.07(1 \mathrm{H}, \mathrm{d}, J=14.0 \mathrm{~Hz}), 4.24(1 \mathrm{H}, \mathrm{d}, J=11.0 \mathrm{~Hz}), 4.33(1 \mathrm{H}, \mathrm{d}, J=11.8 \mathrm{~Hz}), 4.43$ $(1 \mathrm{H}, \mathrm{d}, J=11.8 \mathrm{~Hz}), 4.70(1 \mathrm{H}, \mathrm{d}, J=11.1 \mathrm{~Hz}), 4.82(1 \mathrm{H}, \mathrm{brs}), 6.90(1 \mathrm{H}, \mathrm{s}), 7.00(1 \mathrm{H}, \mathrm{s}), 7.25-7.37$ $(10 \mathrm{H}, \mathrm{m}), 7.47(1 \mathrm{H}, \mathrm{s}) .{ }^{13} \mathrm{C} \mathrm{NMR}\left(125 \mathrm{MHz} \mathrm{CDCl}_{3}\right): \delta=22.1,26.4,31.4,34.6,40.0,49.0,51.4$, 70.2, 73.6, 75.6, 79.8, 121.4, 127.9, 128.6, 128.9, 137.3, 137.9. Found: C, 74.65; H, 7.93; N, 6.48. Anal. Calcd for $\mathrm{C}_{27} \mathrm{H}_{34} \mathrm{~N}_{2} \mathrm{O}_{3}:$ C, 74.62; $\mathrm{H}, 7.89 ; \mathrm{N}, 6.45$.

(R)-1-(Benzyloxy)-2-((1R,2R,4R)-2-(benzyloxy)-4-methylcyclohexyl)-3-(1H-imidazol-1yl)propan-2-ol (39b)

Prepared with 35a and imidazole at reflux for $48 \mathrm{~h}$. Yield: $83 \%$, colorless oil. $[\alpha]_{\mathrm{D}}^{20}=-48.0$ (c $0.285, \mathrm{MeOH}) .{ }^{1} \mathrm{H} \mathrm{NMR}\left(500 \mathrm{MHz}, \mathrm{CDCl}_{3}\right): \delta=0.76-0.95(3 \mathrm{H}, \mathrm{m}), 0.93(1 \mathrm{H}, \mathrm{d}, J=6.5 \mathrm{~Hz})$, 1.38-1.50 (3H, m), $1.65(1 \mathrm{H}, \mathrm{d}, J=13.2 \mathrm{~Hz}), 1.73(1 \mathrm{H}, \mathrm{d}, J=10.2 \mathrm{~Hz}), 1.96(2 \mathrm{H}, \mathrm{brs}), 2.20(1 \mathrm{H}, \mathrm{d}$, $J=12.3 \mathrm{~Hz}), 3.11(1 \mathrm{H}, \mathrm{d}, J=9.7 \mathrm{~Hz}), 3.32(1 \mathrm{H}, \mathrm{d}, J=9.7 \mathrm{~Hz}), 3.54(1 \mathrm{H}, \mathrm{td}, J=10.2,3.8 \mathrm{~Hz}), 3.96$ $(1 \mathrm{H}, \mathrm{d}, J=14.0 \mathrm{~Hz}), 4.12(1 \mathrm{H}, \mathrm{d}, J=14.1 \mathrm{~Hz}), 4.16(1 \mathrm{H}, \mathrm{d}, J=11.0 \mathrm{~Hz}), 4.34(1 \mathrm{H}, \mathrm{d}, J=11.9 \mathrm{~Hz})$, $4.49(1 \mathrm{H}, \mathrm{d}, J=11.9 \mathrm{~Hz}), 4.56(1 \mathrm{H}, \mathrm{d}, J=11.0 \mathrm{~Hz}), 5.17(1 \mathrm{H}, \mathrm{s}), 7.00(1 \mathrm{H}, \mathrm{s}), 7.01(1 \mathrm{H}, \mathrm{s}), 7.21-7.38$ $(10 \mathrm{H}, \mathrm{m}), 7.55(1 \mathrm{H}, \mathrm{s}) .{ }^{13} \mathrm{C}$ NMR $\left(125 \mathrm{MHz}, \mathrm{CDCl}_{3}\right): \delta=22.1,26.7,31.4,34.6,39.8,48.1,52.2$, 70.1, 72.1, 73.7, 75.3, 80.5, 121.3, 128.2, 128.6, 128.8, 137.2, 137.9, 138.7. Found: C, 74.60; H, 7.87; $\mathrm{N}, 6.50$. Anal. Calcd for $\mathrm{C}_{27} \mathrm{H}_{34} \mathrm{~N}_{2} \mathrm{O}_{3}$ : C, 74.62; $\mathrm{H}, 7.89 ; \mathrm{N}, 6.45$.

(S)-1-(Benzyloxy)-2-((1R,2R,4R)-2-(benzyloxy)-4-methylcyclohexyl)-3-(1H-1,2,4-triazol-1yl)propan-2-ol (40a)

Prepared with 35a and 1,2,4-triazole at reflux for $48 \mathrm{~h}$. Yield: 83\%, colorless oil. $[\alpha]_{\mathrm{D}}^{20}=-58.0(\mathrm{c} 0.265, \mathrm{MeOH}) .{ }^{1} \mathrm{H}$ NMR $\left(500 \mathrm{MHz}, \mathrm{CDCl}_{3}\right): \delta=0.84-0.97(2 \mathrm{H}, \mathrm{m}), 0.94$ $(3 \mathrm{H}, \mathrm{d}, J=6.5 \mathrm{~Hz}), 1.04-1.13(1 \mathrm{H}, \mathrm{m}), 1.38-1.45(1 \mathrm{H}, \mathrm{m}), 1.67(1 \mathrm{H}, \mathrm{d}, J=12.8 \mathrm{~Hz}), 1.78(1 \mathrm{H}$, $\mathrm{td}, J=12.5,3.2 \mathrm{~Hz}), 1.90-1.94(1 \mathrm{H}, \mathrm{m}), 2.25(1 \mathrm{H}, \mathrm{d}, J=12.3 \mathrm{~Hz}), 2.59(2 \mathrm{H}, \mathrm{s}), 3.18(1 \mathrm{H}, \mathrm{d}$, $J=10.0 \mathrm{~Hz}), 3.32(1 \mathrm{H}, \mathrm{d}, J=10.0 \mathrm{~Hz}), 3.43(1 \mathrm{H}, \mathrm{td}, J=10.6,3.9 \mathrm{~Hz}), 4.18(1 \mathrm{H}, \mathrm{d}, J=14.1 \mathrm{~Hz})$, $4.20(1 \mathrm{H}, \mathrm{d}, J=10.9 \mathrm{~Hz}), 4.33(1 \mathrm{H}, \mathrm{d}, J=14.0 \mathrm{~Hz}), 4.40(1 \mathrm{H}, \mathrm{d}, J=11.9 \mathrm{~Hz}), 4.50(1 \mathrm{H}, \mathrm{d}$, $J=11.9 \mathrm{~Hz}), 4.64(1 \mathrm{H}, \mathrm{d}, J=10.9 \mathrm{~Hz}), 5.02(1 \mathrm{H}, \mathrm{brs}), 7.25-7.36(10 \mathrm{H}, \mathrm{m}), 7.88(1 \mathrm{H}, \mathrm{s}), 7.97$ $(1 \mathrm{H}, \mathrm{s}) .{ }^{13} \mathrm{C}$ NMR $\left(125 \mathrm{MHz}, \mathrm{CDCl}_{3}\right): \delta=22.1,26.1,31.4,34.5,40.1,47.7,54.3,70.1,73.1$, $73.8,75.9,79.5,127.9,128.0,128.4,128.5,128.6,128.9,137.5,138.0,150.5$. Found: $\mathrm{C}, 71.69 ; \mathrm{H}$, 7.67; N, 9.66. Anal. Calcd for $\mathrm{C}_{26} \mathrm{H}_{33} \mathrm{~N}_{3} \mathrm{O}_{3}$ : C, 71.70; $\mathrm{H}, 7.64 ; \mathrm{N}, 9.65$.

(R)-1-(Benzyloxy)-2-((1R,2R,4R)-2-(benzyloxy)-4-methylcyclohexyl)-3-(1H-1,2,4-triazol-1yl)propan-2-ol (40b)

Prepared with 35a and 1,2,4-triazole at reflux for $48 \mathrm{~h}$. Yield: 83\%, colorless oil. $[\alpha]_{\mathrm{D}}^{20}=-57.0$ (c 0.265, MeOH). ${ }^{1} \mathrm{H}$ NMR $\left(500 \mathrm{MHz}, \mathrm{CDCl}_{3}\right): \delta=0.74-0.95(3 \mathrm{H}, \mathrm{m}), 0.91(3 \mathrm{H}$, $\mathrm{d}, J=6.5 \mathrm{~Hz}), 1.17-1.42(6 \mathrm{H}, \mathrm{m}), 1.64(1 \mathrm{H}, \mathrm{d}, J=13.7 \mathrm{~Hz}), 2.06(1 \mathrm{H}, \mathrm{d}, J=10.1 \mathrm{~Hz}), 2.18$ $(1 \mathrm{H}, \mathrm{d}, J=12.2 \mathrm{~Hz}), 3.28(1 \mathrm{H}, \mathrm{d}, J=9.8 \mathrm{~Hz}), 3.41(1 \mathrm{H}, \mathrm{d}, J=9.7 \mathrm{~Hz}), 3.59(1 \mathrm{H}, \mathrm{td}, J=10.2$, $3.8 \mathrm{~Hz}), 4.19(1 \mathrm{H}, \mathrm{d}, J=10.9 \mathrm{~Hz}), 4.27(1 \mathrm{H}, \mathrm{d}, J=14.3 \mathrm{~Hz}), 4.40(1 \mathrm{H}, \mathrm{d}, J=14.3 \mathrm{~Hz}), 4.41$ $(1 \mathrm{H}, \mathrm{d}, J=12.0 \mathrm{~Hz}), 4.55(1 \mathrm{H}, \mathrm{d}, J=11.9 \mathrm{~Hz}), 456(1 \mathrm{H}, \mathrm{d}, J=10.9 \mathrm{~Hz}), 5.35(1 \mathrm{H}, \mathrm{s}), 7.21-7.37$ $(10 \mathrm{H}, \mathrm{m}), 7.89(1 \mathrm{H}, \mathrm{s}), 8.28(1 \mathrm{H}, \mathrm{s}) .{ }^{13} \mathrm{C} \mathrm{NMR}\left(125 \mathrm{MHz}, \mathrm{CDCl}_{3}\right): \delta=22.1,26.6,31.4,34.5$, 39.9, 47.4, 54.8, 70.1, 72.6, 73.8, 75.8, 80.4, 128.0, 128.1, 128.2, 128.4, 128.7, 137.3, 138.0, 150.7. Found: $\mathrm{C}, 71.73 ; \mathrm{H}, 7.60 ; \mathrm{N}, 9.62$. Anal. Calcd for $\mathrm{C}_{26} \mathrm{H}_{33} \mathrm{~N}_{3} \mathrm{O}_{3}: \mathrm{C}, 71.70 ; \mathrm{H}, 7.64 ; \mathrm{N}, 9.65$. 
(1R,2R,5R)-2-((S)-1-(Benzyloxy)-2-hydroxy-3-(1H-imidazol-1-yl)propan-2-yl)-5methylcyclohexanol (42a)

Prepared with $35 \mathrm{~b}$ and imidazole at reflux for $12 \mathrm{~h}$. Yield: 58\%, white crystal, m.p. $=133-134{ }^{\circ} \mathrm{C} .[\alpha]_{\mathrm{D}}^{20}=-22.0$ (c 0.25, MeOH). ${ }^{1} \mathrm{H}$ NMR $\left(500 \mathrm{MHz}, \mathrm{CDCl}_{3}\right): \delta=0.84-0.92$ $(1 \mathrm{H}, \mathrm{m}), 0.92(3 \mathrm{H}, \mathrm{d}, J=6.5 \mathrm{~Hz}), 0.97-1.07(2 \mathrm{H}, \mathrm{m}), 1.39-1.47(1 \mathrm{H}, \mathrm{m}), 1.65-1.77(2 \mathrm{H}, \mathrm{m})$, 1.84-1.88 (1H, m), 1.94-1.97 (H, m), $3.16(1 \mathrm{H}, \mathrm{d}, J=9.5 \mathrm{~Hz}), 3.28(1 \mathrm{H}, \mathrm{d}, J=9.5 \mathrm{~Hz}), 3.64$ $(1 \mathrm{H}, \mathrm{td}, J=10.5,4.2 \mathrm{~Hz}), 4.11(1 \mathrm{H}, \mathrm{d}, J=14.3 \mathrm{~Hz}), 4.20(1 \mathrm{H}, \mathrm{d}, J=14.2 \mathrm{~Hz}), 4.38(1 \mathrm{H}, \mathrm{d}$, $J=11.8 \mathrm{~Hz}), 4.48(1 \mathrm{H}, \mathrm{d}, J=11.8 \mathrm{~Hz}), 7.00(2 \mathrm{H}, \mathrm{s}), 7.25-7.35(5 \mathrm{H}, \mathrm{m}), 7.53(1 \mathrm{H}, \mathrm{s}) .{ }^{13} \mathrm{C}$ NMR $\left(125 \mathrm{MHz}, \mathrm{CDCl}_{3}\right): \delta=21.9,26.2,31.4,34.6,45.5,49.7,50.6,72.0,73.3,73.7,76.5,121.2$, $127.9,128.1,128.6,128.7,137.7,138.8$. Found: C, 69.71; H, 8.16; N, 8.15. Anal. Calcd for $\mathrm{C}_{20} \mathrm{H}_{28} \mathrm{~N}_{2} \mathrm{O}_{3}: \mathrm{C}, 69.74 ; \mathrm{H}, 8.19 ; \mathrm{N}, 8.13$.

$(1 R, 2 R, 5 R)-2-((R)-1-($ Benzyloxy)-2-hydroxy-3-(1H-imidazol-1-yl)propan-2-yl)-5methylcyclohexanol (42b)

Prepared with $35 \mathrm{~b}$ and imidazole at reflux for $12 \mathrm{~h}$. Yield: $58 \%$, colorless oil. $[\alpha]_{\mathrm{D}}^{20}=-8.0$ (c $0.25, \mathrm{MeOH}) .{ }^{1} \mathrm{H}$ NMR $\left(500 \mathrm{MHz}, \mathrm{CDCl}_{3}\right): \delta=0.77(1 \mathrm{H}, \mathrm{q}, J=11.6 \mathrm{~Hz}), 0.89(3 \mathrm{H}, \mathrm{d}$, $J=6.4 \mathrm{~Hz}), 0.99(1 \mathrm{H}, \mathrm{d}, J=11.6 \mathrm{~Hz}), 1.26-1.42(3 \mathrm{H}, \mathrm{m}), 1.64(1 \mathrm{H}, \mathrm{d}, J=13.3 \mathrm{~Hz}), 1.69(1 \mathrm{H}, \mathrm{d}$, $J=8.5 \mathrm{~Hz}), 1.91(1 \mathrm{H}, \mathrm{d}, J=12.1 \mathrm{~Hz}), 3.38(1 \mathrm{H}, \mathrm{d}, J=9.7 \mathrm{~Hz}), 3.57(1 \mathrm{H}, \mathrm{d}, J=9.6 \mathrm{~Hz}), 3.80(1 \mathrm{H}, \mathrm{t}$, $J=7.4 \mathrm{~Hz}), 4.02(1 \mathrm{H}, \mathrm{d}, J=14.2 \mathrm{~Hz}), 4.16(1 \mathrm{H}, \mathrm{d}, J=14.2 \mathrm{~Hz}), 4.49(1 \mathrm{H}, \mathrm{d}, J=11.9 \mathrm{~Hz}), 4.55(1 \mathrm{H}$, $\mathrm{d}, J=11.8 \mathrm{~Hz}), 6.98(1 \mathrm{H}, \mathrm{s}), 6.99(1 \mathrm{H}, \mathrm{s}), 7.25-7.38(5 \mathrm{H}, \mathrm{m}), 7.52(1 \mathrm{H}, \mathrm{s}) .{ }^{13} \mathrm{C} \mathrm{NMR}(125 \mathrm{MHz}$, $\left.\mathrm{CDCl}_{3}\right): \delta=21.9,26.4,31.5,34.5,45.3,48.3,52.4,72.2,72.5,73.9,76.3,121.1,127.9,128.1,128.3$, 128.7, 137.8, 138.7. Found: C, 69.77; $\mathrm{H}, 8.17 ; \mathrm{N}, 8.10$. Anal. Calcd for $\mathrm{C}_{20} \mathrm{H}_{28} \mathrm{~N}_{2} \mathrm{O}_{3}: \mathrm{C}, 69.74 ; \mathrm{H}$, $8.19 ; \mathrm{N}, 8.13$.

$(1 R, 2 R, 5 R)-2-((S)-1-($ Benzyloxy)-2-hydroxy-3-(1H-1,2,4-triazol-1-yl)propan-2-yl)-5methylcyclohexanol (43a)

Prepared with $\mathbf{3 5 b}$ and 1,2,4-triazole at reflux for $12 \mathrm{~h}$. Yield: 67\%, white crystal, m.p. $=53-54{ }^{\circ} \mathrm{C} .[\alpha]_{\mathrm{D}}^{20}=-16.0(\mathrm{c} 0.25, \mathrm{MeOH}) .{ }^{1} \mathrm{H} \mathrm{NMR}\left(500 \mathrm{MHz}, \mathrm{CDCl}_{3}\right): \delta=0.80-0.89$ $(1 \mathrm{H}, \mathrm{m}), 0.91(1 \mathrm{H}, \mathrm{d}, J=6.5 \mathrm{~Hz}), 0.96-1.07(2 \mathrm{H}, \mathrm{m}), 1.21-1.46(3 \mathrm{H}, \mathrm{m}), 1.62-1.68(2 \mathrm{H}, \mathrm{m})$, 181-1.85 (1H, m), 1.95-1.99 (1H, m), $3.27(1 \mathrm{H}, \mathrm{d}, J=9.7 \mathrm{~Hz}), 3.34(1 \mathrm{H}, \mathrm{d}, J=9.7 \mathrm{~Hz}), 3.58$ $(1 \mathrm{H}, \mathrm{td}, J=10.6,4.1 \mathrm{~Hz}), 4.37-4.53(4 \mathrm{H}, \mathrm{m}), 7.25-7.36(5 \mathrm{H}, \mathrm{m}), 7.91(1 \mathrm{H}, \mathrm{s}), 8.17(1 \mathrm{H}, \mathrm{s}) .{ }^{13} \mathrm{C}$ $\operatorname{NMR}\left(125 \mathrm{MHz}, \mathrm{CDCl}_{3}\right): \delta=22.0,25.8,31.4,34.5,45.4,48.9,53.6,71.7,72.8,73.8,76.5,127.9$, $128.1,128.6,137.5,151.3$. Found: $\mathrm{C}, 66.10 ; \mathrm{H}, 7.85 ; \mathrm{N}, 12.12$. Anal. Calcd for $\mathrm{C}_{19} \mathrm{H}_{27} \mathrm{~N}_{3} \mathrm{O}_{3}$ : C, 66.06; H, 7.88; N, 12.16 .

$(1 R, 2 R, 5 R)-2-((R)-1-(B e n z y l o x y)-2-h y d r o x y-3-(1 \mathrm{H}-1,2,4-$ triazol-1-yl)propan-2-yl)-5methylcyclohexanol (43b)

Prepared with 35b and 1,2,4-triazole at reflux for $12 \mathrm{~h}$. Yield: 58\%, colorless oil. $[\alpha]_{\mathrm{D}}^{20}=-6.0$ (c 0.25, MeOH). ${ }^{1} \mathrm{H}$ NMR $\left(500 \mathrm{MHz} \mathrm{CDCl}_{3}\right): \delta=0.71-0.79(1 \mathrm{H}, \mathrm{m}), 0.88(1 \mathrm{H}$, $\mathrm{d}, J=6.5 \mathrm{~Hz}), 0.93(1 \mathrm{H}, \mathrm{q}, J=12.0 \mathrm{~Hz}), 1.26-1.29(2 \mathrm{H}, \mathrm{m}), 1.36-1.43(1 \mathrm{H}, \mathrm{m}), 1.63(1 \mathrm{H}, \mathrm{d}$, $J=13.2 \mathrm{~Hz}), 1.88-1.94(2 \mathrm{H}, \mathrm{m}), 3.45(1 \mathrm{H}, \mathrm{d}, J=9.8 \mathrm{~Hz}), 3.61(1 \mathrm{H}, \mathrm{d}, J=9.8 \mathrm{~Hz}), 3.79(1 \mathrm{H}, \mathrm{td}$, $J=10.4,4.1 \mathrm{~Hz}), 4.37(1 \mathrm{H}, \mathrm{d}, J=14.3 \mathrm{~Hz}), 4.41(1 \mathrm{H}, \mathrm{d}, J=14.3 \mathrm{~Hz}), 4.50(1 \mathrm{H}, \mathrm{d}, J=11.9 \mathrm{~Hz})$, $4.56(1 \mathrm{H}, \mathrm{d}, J=11.9 \mathrm{~Hz}), 7.26-7.37(5 \mathrm{H}, \mathrm{m}), 7.89(1 \mathrm{H}, \mathrm{s}), 8.21(1 \mathrm{H}, \mathrm{s}) .{ }^{13} \mathrm{C}$ NMR $(125 \mathrm{MHz}$, $\left.\mathrm{CDCl}_{3}\right): \delta=21.9,26.2,31.4,34.5,45.2,48.2,54.7,72.2,74.0,127.9,128.1,128.6,137.7,145.0$, 151.1. Found: $\mathrm{C}, 66.03 ; \mathrm{H}, 7.90 ; \mathrm{N}, 12.19$. Anal. Calcd for $\mathrm{C}_{19} \mathrm{H}_{27} \mathrm{~N}_{3} \mathrm{O}_{3}: \mathrm{C}, 66.06 ; \mathrm{H}, 7.88 ; \mathrm{N}$, 12.16 .

\subsubsection{General Procedure for Debenzylation}

A suspension of palladium-on-carbon ( $5 \% \mathrm{Pd} / \mathrm{C}, 0.22 \mathrm{~g})$ in $\mathrm{MeOH}(50 \mathrm{~mL})$ was added to (+)-neoisopulegol-based O-benzyl derivatives $(14.0 \mathrm{mmol})$ in $\mathrm{MeOH}(100 \mathrm{~mL})$ and the mixture was stirred under a $\mathrm{H}_{2}$ atmosphere $(1 \mathrm{~atm})$ at room temperature. After completion 
of the reaction (as monitored by TLC, $24 \mathrm{~h}$ ), the mixture was filtered through a Celite pad and the solution was evaporated to dryness. The crude products were recrystallized in diethyl ether, resulting in primary aminodiols $(\mathbf{9 a}-\mathbf{b})$ and aminotriols $(\mathbf{1 6} \mathbf{a}-\mathbf{b})$.

(1S,2R,5R)-2-((R)-1-Amino-2-hydroxypropan-2-yl)-5-methylcyclohexanol (9a)

Prepared with 5a. Yield: $91 \%$, white crystal, m.p. $=100-110{ }^{\circ} \mathrm{C}[\alpha]_{\mathrm{D}}^{20}=+14.0(\mathrm{c} 0.25$, $\mathrm{MeOH}) .{ }^{1} \mathrm{H}$ NMR $\left(500 \mathrm{MHz}\right.$, DMSO- $\left.d_{6}\right): \delta=0.75-0.85(2 \mathrm{H}, \mathrm{m}), 0.80(3 \mathrm{H}, \mathrm{d}, J=5.3 \mathrm{~Hz}), 0.99$ $(1 \mathrm{H}, \mathrm{d}, J=12.1 \mathrm{~Hz}), 1.17(3 \mathrm{H}, \mathrm{s}), 1.31(1 \mathrm{H}, \mathrm{d}, J=11.7 \mathrm{~Hz}), 1.45(1 \mathrm{H}, \mathrm{q}, J=10.9 \mathrm{~Hz}), 1.58(1 \mathrm{H}$, $\mathrm{d}, J=10.4 \mathrm{~Hz}), 1.65-1.80(3 \mathrm{H}, \mathrm{m}), 2.70(1 \mathrm{H}, \mathrm{d}, J=12.7 \mathrm{~Hz}), 2.89(1 \mathrm{H}, \mathrm{d}, J=12.7 \mathrm{~Hz}), 4.04$ $(1 \mathrm{H}, \mathrm{s}), 4.95(1 \mathrm{H}, \mathrm{brs}) .{ }^{13} \mathrm{C}$ NMR $\left(125 \mathrm{MHz}, \mathrm{DMSO}-d_{6}\right): \delta=20.1,22.2,23.3,25.4,34.7,42.8$, $45.9,49.0,65.1,71.3$. Found: $\mathrm{C}, 64.09 ; \mathrm{H}, 11.35 ; \mathrm{N}, 7.50$. Anal. Calcd for $\mathrm{C}_{10} \mathrm{H}_{21} \mathrm{NO}_{2}: \mathrm{C}$, 64.13; H, 11.30; N, 7.48.

(1S,2R,5R)-2-((S)-1-Amino-2-hydroxypropan-2-yl)-5-methylcyclohexanol (9b)

Prepared with 5b. Yield: $91 \%$, white crystal, m.p. $=138-140{ }^{\circ} \mathrm{C} .[\alpha]_{\mathrm{D}}^{20}=+10.0(\mathrm{c} 0.25$, $\mathrm{MeOH}) .{ }^{1} \mathrm{H}$ NMR $\left(500 \mathrm{MHz}, \mathrm{DMSO}-d_{6}\right): \delta=0.82(3 \mathrm{H}, \mathrm{d}, J=5.7 \mathrm{~Hz}), 0.81-0.88(1 \mathrm{H}, \mathrm{m}), 1.02$ $(1 \mathrm{H}, \mathrm{t}, J=12.5 \mathrm{~Hz}), 1.17(3 \mathrm{H}, \mathrm{s}), 1.32(1 \mathrm{H}, \mathrm{d}, J=10.2 \mathrm{~Hz}), 1.45-1.55(2 \mathrm{H}, \mathrm{m}), 1.65-1.80(3 \mathrm{H}$, $\mathrm{m}), 2.62(1 \mathrm{H}, \mathrm{d}, J=12.7 \mathrm{~Hz}), 2.91(1 \mathrm{H}, \mathrm{d}, J=12.8 \mathrm{~Hz}), 4.12(1 \mathrm{H}, \mathrm{s}), 4.86(1 \mathrm{H}, \mathrm{brs}), 6.85(3 \mathrm{H}$, brs). ${ }^{13} \mathrm{C}$ NMR (125 MHz, DMSO- $\left.d_{6}\right): \delta=20.7,22.0,25.1,25.2,34.7,42.4,45.2,49.5,64.3$, 70.9. Found: $\mathrm{C}, 64.15 ; \mathrm{H}, 11.27 ; \mathrm{N}, 7.45$. Anal. Calcd for $\mathrm{C}_{10} \mathrm{H}_{21} \mathrm{NO}_{2}: \mathrm{C}, 64.13 ; \mathrm{H}, 11.30 ; \mathrm{N}$, 7.48.

(S)-3-Amino-2-((1R,2S,4R)-2-hydroxy-4-methylcyclohexyl)propane-1,2-diol (16a)

Prepared with 12a, 20a or 25a. Yield: 78\% (12a), 94\% (20a), 91\% (25a), white crystal, m.p. $=107-106{ }^{\circ} \mathrm{C} .[\alpha]_{\mathrm{D}}^{20}=+18.0$ (c $\left.0.30, \mathrm{MeOH}\right) .{ }^{1} \mathrm{H}$ NMR $\left(500 \mathrm{MHz}, \mathrm{DMSO}-d_{6}\right): \delta=0.80$ $(3 \mathrm{H}, \mathrm{d}, J=6.6 \mathrm{~Hz}), 0.79-0.90(1 \mathrm{H}, \mathrm{m}), 0.93-1.00(1 \mathrm{H}, \mathrm{m}), 1.38-1.41(1 \mathrm{H}, \mathrm{m}), 1.45-1.54(2 \mathrm{H}$, $\mathrm{m}), 1.60-1.70(2 \mathrm{H}, \mathrm{m}), 1.73-1.85(1 \mathrm{H}, \mathrm{m}), 2.60(1 \mathrm{H}, \mathrm{d}, J=12.6 \mathrm{~Hz}), 3.30(2 \mathrm{H}, \mathrm{q}, J=10.9 \mathrm{~Hz})$, $4.07(1 \mathrm{H}, \mathrm{s}) .{ }^{13} \mathrm{C}$ NMR $\left(125 \mathrm{MHz}, \mathrm{DMSO}-d_{6}\right): \delta=20.3,22.4,25.4,35.1,42.3,44.1,45.2,64.4$, 65.2, 74.9. Found: $\mathrm{C}, 59.10 ; \mathrm{H}, 10.38 ; \mathrm{N}, 6.93$. Anal. Calcd for $\mathrm{C}_{10} \mathrm{H}_{21} \mathrm{NO}_{3}: \mathrm{C}, 59.08 ; \mathrm{H}, 10.41$; $\mathrm{N}, 6.89$.

(R)-3-Amino-2-((1R,2S,4R)-2-hydroxy-4-methylcyclohexyl)propane-1,2-diol (16b)

Prepared with 20b or 25b. Yield: $94 \%(\mathbf{2 0 b}), 91 \%(\mathbf{2 5 b})$, white crystal, m.p. $=80-82{ }^{\circ} \mathrm{C}$. $[\alpha]_{\mathrm{D}}^{20}=+13.0(\mathrm{c} 0.30, \mathrm{MeOH}) .{ }^{1} \mathrm{H}$ NMR $\left(500 \mathrm{MHz}, \mathrm{DMSO}-d_{6}\right): \delta=0.80(3 \mathrm{H}, \mathrm{d}, J=6.6 \mathrm{~Hz})$, $0.82-0.88(1 \mathrm{H}, \mathrm{m}), 0.94-0.99(1 \mathrm{H}, \mathrm{m}), 1.44-1.57(3 \mathrm{H}, \mathrm{m}), 1.64-1.69(2 \mathrm{H}, \mathrm{m}), 1.73-1.77(1 \mathrm{H}$, $\mathrm{m}), 2.57(2 \mathrm{H}, \mathrm{q}, J=12.7 \mathrm{~Hz}), 3.32(1 \mathrm{H}, \mathrm{d}, J=11.0 \mathrm{~Hz}), 3.39(1 \mathrm{H}, \mathrm{d}, J=11.0 \mathrm{~Hz}), 4.00(1 \mathrm{H}$, s). ${ }^{13} \mathrm{C}$ NMR $\left(125 \mathrm{MHz}\right.$, DMSO- $\left.d_{6}\right): \delta=19.8,22.4,25.4,35.1,42.4,44.7,45.3,64.2,64.8,75.3$. Found: $\mathrm{C}, 59.05 ; \mathrm{H}, 10.43 ; \mathrm{N}, 6.87$. Anal. Calcd for $\mathrm{C}_{10} \mathrm{H}_{21} \mathrm{NO}_{3}$ : $\mathrm{C}, 59.08 ; \mathrm{H}, 10.41 ; \mathrm{N}, 6.89$.

\subsection{General Procedure for Antimicrobial Assays}

For the antimicrobial analyses the pure compounds were first dissolved in $\mathrm{MeOH}$ and diluted with $\mathrm{H}_{2} \mathrm{O}$ to two concentration levels $\left(400 \mu \mathrm{g} \mathrm{mL}^{-1}\right.$ and $\left.40 \mu \mathrm{g} \mathrm{mL}^{-1}\right)$ keeping the final $\mathrm{MeOH}$ content at $10 \%$. Then these solutions were investigated in microdilution assay with two Gram-positive bacteria including Bacillus subtilis SZMC 0209 and Staphylococcus aureus SZMC 14611, two Gram-negative bacteria Escherichia coli SZMC 6271 and Pseudomonas aeruginosa SZMC 23290, as well as two yeast strains Candida albicans SZMC 1533 and C. krusei SZMC 1352 according to the M07-A10 CLSI guideline [92] and our previous work [93]. Suspensions of the test microbes were prepared from overnight cultures cultivated in ferment broth (bacteria: $10 \mathrm{~g} \mathrm{~L}^{-1}$ peptone, $5 \mathrm{~g} \mathrm{~L}^{-1} \mathrm{NaCl}, 5 \mathrm{~g} \mathrm{~L}^{-1}$ yeast extract; yeast: $20 \mathrm{~g} \mathrm{~L}^{-1}$ peptone, $10 \mathrm{~g} \mathrm{~L}^{-1}$ yeast extract, $20 \mathrm{~g} \mathrm{~L}^{-1}$ glucose) at $37^{\circ} \mathrm{C}$. Then the concentrations of the suspensions were set to $2 \times 10^{5}$ cells mL $\mathrm{mL}^{-1}$ with sterile media. For the assay, 96-well plates were prepared by dispensing into each well $100 \mu \mathrm{L}$ of suspension containing the bacterial or yeast cells and $50 \mu \mathrm{L}$ of sterile broth as well as $50 \mu \mathrm{L}$ of the test solutions and incubated for $24 \mathrm{~h}$ at $37^{\circ} \mathrm{C}$. The mixture of $150 \mu \mathrm{L}$ broth 
and $50 \mu \mathrm{L}$ of $10 \%$ methanol was used as the blank sample for the background correction, while $100 \mu \mathrm{L}$ of microbial suspension supplemented with $50 \mu \mathrm{L}$ sterile broth and $50 \mu \mathrm{L}$ of $10 \%$ methanol was applied as negative control. The positive control contained ampicillin (Sigma) or nystatin (Sigma) for bacteria or fungi, respectively, at two final concentration levels $\left(100 \mu \mathrm{g} \mathrm{mL}^{-1}\right.$ and $\left.10 \mu \mathrm{g} \mathrm{mL}^{-1}\right)$. The inhibitory effects of the derivatives were observed spectrophotometrically at $620 \mathrm{~nm}$ after the incubation, and inhibition was calculated as the percentage of the positive control after blank correction.

The MIC was also determined for certain compounds, which were based on the broth microdilution method described above and in the M07-A10 CLSI guideline [92]. The compounds were prepared in two-fold dilutions in $10 \% \mathrm{MeOH}$ covering the final concentration range of $0.78-100.00 \mu \mathrm{g} / \mathrm{mL}$. The MIC was observed as the lowest concentration level of the compound that completely inhibits the growth of the organism in microdilution wells as detected by the unaided eye. All experiments were repeated three times.

\section{Conclusions}

The results of the present study establishing antimicrobial and antifungal behavior of some synthetic derivatives are promising with respect to possible clinical application. It is strongly believed that it will serve a suitable basis for future research on developing alternative antibiotics focusing on the development of better antibiotics against infectious organisms. The obtained results indicate that the di-O-benzyl derivatives may have considerable potential for therapeutic application as novel drug candidates against bacterial and fungal infections. Based on the results obtained, some of the studied compounds have proved to be promising candidates for additional efficacy evaluation.

Furthermore, in vitro studies have clearly shown that the $O$-benzyl substituent on the cyclohexyl ring in aminodiol and aminotriol derivatives is essential to have an antimicrobial effect whereas the stereochemistry of the $O$-benzyl substituent on the cyclohexane ring in the aminodiol and aminotriol function has no influence on the antimicrobial effect.

In addition, the antifungal activity was found to be affected by the stereochemistry of the derivatives, namely the $S$-isomers were more potent than the corresponding $R$ isomers against fungi while the antibacterial effect did not distinguish between the different stereoisomers.

In the next stage of our project, we plan to obtain $N$-benzyl and imidazole $O$-benzyl analogs, preferably different substitutions on $N$-benzyl and imidazole systems, to increase their antimicrobial activities on various microorganisms. For the optimized compounds, additionally, docking studies and molecular dynamics study will also be performed to get an insight into the dynamics of ligand interaction.

Supplementary Materials: The following are available online at https:/ / www.mdpi.com/article/10 $.3390 /$ ijms22115626/s1.

Author Contributions: Z.S. and A.S. conceived and designed the experiments; T.M.L., T.H. and F.Z.B. performed the experiments, analyzed the data, and wrote the experimental part; Z.S., F.F. and A.S. discussed the results and contributed to manuscript writing. All authors have read and agreed to the published version of the manuscript.

Funding: This research was funded by EU-funded Hungarian grant, grant number GINOP-2.3.2-152016-00012, Ministry of Human Capacities, Hungary grant 20391-3/2018/FEKUSTRAT, Hungarian Research Foundation (OTKA No. K 115731). The APC was funded by University of Szeged Open Access Fund (No. 5315) and Hungarian Academy of Science.

Acknowledgments: We are grateful for financial supports from the EU-funded Hungarian grant GINOP-2.3.2-15-2016-00012, Ministry of Human Capacities, Hungary grant 20391-3/2018/FEKUSTRAT, Hungarian Research Foundation (OTKA No. K 115731).

Conflicts of Interest: The authors declare no conflict of interest. The funders had no role in the design of the study; in the collection, analyses, or interpretation of data; in the writing of the manuscript, or in the decision to publish the results. 


\section{References}

1. Dabholkar, V.V.; Ansari, F.Y. Novel Pyrimidine Derivatives by Sonication and Traditional Thermal Methods. Green Chem. Lett. Rev. 2010, 3, 245-248. [CrossRef]

2. Koparir, M.; Orek, C.; Parlak, A.E.; Söylemez, A.; Koparir, P.; Karatepe, M.; Dastan, S.D. Synthesis and Biological Activities of Some Novel Aminomethyl Derivatives of 4-Substituted-5-(2-Thienyl)-2,4-Dihydro-3H-1,2,4-Triazole-3-Thiones. Eur. J. Med. Chem. 2013, 63, 340-346. [CrossRef]

3. Romero, D.H.; Heredia, V.E.T.; García-Barradas, O.; López, M.E.M.; Pavón, E.S. Synthesis of Imidazole Derivatives and Their Biological Activities. J. Chem. Biochem. 2014, 2. [CrossRef]

4. Kumar, S.S.; Kavitha, H.P. Synthesis and Biological Applications of Triazole Derivatives-A Review. Mini-Rev. Org. Chem. 2013, 10, 40-65. [CrossRef]

5. Behrouz, S.; Rad, M.N.S.; Rostami, S.; Behrouz, M.; Zarehnezhad, E.; Zarehnezhad, A. Design, Synthesis, and Biological Activities of Novel Azole-Bonded $\beta$-Hydroxypropyl Oxime O-Ethers. Mol. Divers. 2014, 18, 797-808. [CrossRef] [PubMed]

6. Kosmalski, T.; Kutkowska, J.; Dwojak, I.; Studzińska, R.; Sikora, A.; Modzelewska-Banachiewicz, B.; Gzella, A. Novel O-Benzyl Oxime Ethers of 1-(Thiophen-2-Yl)Ethan-1-One-Synthesis, Structure and Antimicrobial Activity. Heterocycles 2017, $94,523$. [CrossRef]

7. Padmavathi, V.; Thriveni, P.; Sudhakar Reddy, G.; Deepti, D. Synthesis and Antimicrobial Activity of Novel Sulfone-Linked Bis Heterocycles. Eur. J. Med. Chem. 2008, 43, 917-924. [CrossRef]

8. Sztanke, K.; Tuzimski, T.; Rzymowska, J.; Pasternak, K.; Kandefer-Szerszeń, M. Synthesis, Determination of the Lipophilicity, Anticancer and Antimicrobial Properties of Some Fused 1,2,4-Triazole Derivatives. Eur. J. Med. Chem. 2008, 43, 404-419. [CrossRef] [PubMed]

9. Buzdar, A.U.; Robertson, J.F.R.; Eiermann, W.; Nabholtz, J.-M. An Overview of the Pharmacology and Pharmacokinetics of the Newer Generation Aromatase Inhibitors Anastrozole, Letrozole, and Exemestane. Cancer 2002, 95, 2006-2016. [CrossRef]

10. Amir, M.; Kumar, H.; Javed, S.A. Condensed Bridgehead Nitrogen Heterocyclic System: Synthesis and Pharmacological Activities of 1,2,4-Triazolo-[3,4-b]-1,3,4-Thiadiazole Derivatives of Ibuprofen and Biphenyl-4-Yloxy Acetic Acid. Eur. J. Med. Chem. 2008, 43, 2056-2066. [CrossRef]

11. Ghannoum, M.A.; Rice, L.B. Antifungal Agents: Mode of Action, Mechanisms of Resistance, and Correlation of These Mechanisms with Bacterial Resistance. Clin. Microbiol. Rev. 1999, 12, 501-517. [CrossRef] [PubMed]

12. Küçükgüzel, Ş.G.; Çıkla-Süzgün, P. Recent Advances Bioactive 1,2,4-Triazole-3-Thiones. Eur. J. Med. Chem. 2015, 97, 830-870. [CrossRef] [PubMed]

13. Kuş, C.; Ayhan-Kılcıgil, G.; Özbey, S.; Kaynak, F.B.; Kaya, M.; Çoban, T.; Can-Eke, B. Synthesis and Antioxidant Properties of Novel N-Methyl-1,3,4-Thiadiazol-2-Amine and 4-Methyl-2H-1,2,4-Triazole-3(4H)-Thione Derivatives of Benzimidazole Class. Bioorg. Med. Chem. 2008, 16, 4294-4303. [CrossRef] [PubMed]

14. Boraei, A.T.A.; Gomaa, M.S.; El Ashry, E.S.H.; Duerkop, A. Design, Selective Alkylation and X-Ray Crystal Structure Determination of Dihydro-Indolyl-1,2,4-Triazole-3-Thione and Its 3-Benzylsulfanyl Analogue as Potent Anticancer Agents. Eur. J. Med. Chem. 2017, 125, 360-371. [CrossRef]

15. Aouad, M.R.; Mayaba, M.M.; Naqvi, A.; Bardaweel, S.K.; Al-blewi, F.F.; Messali, M.; Rezki, N. Design, Synthesis, in Silico and in Vitro Antimicrobial Screenings of Novel 1,2,4-Triazoles Carrying 1,2,3-Triazole Scaffold with Lipophilic Side Chain Tether. Chem. Cent. J. 2017, 11. [CrossRef] [PubMed]

16. Vijesh, A.M.; Isloor, A.M.; Shetty, P.; Sundershan, S.; Fun, H.K. New Pyrazole Derivatives Containing 1,2,4-Triazoles and Benzoxazoles as Potent Antimicrobial and Analgesic Agents. Eur. J. Med. Chem. 2013, 62, 410-415. [CrossRef]

17. Plech, T.; Kaproń, B.; Łuszczki, J.J.; Paneth, A.; Siwek, A.; Kołaczkowski, M.; Żołnierek, M.; Nowak, G. Studies on the Anticonvulsant Activity of 4-Alkyl-1,2,4-Triazole-3-Thiones and Their Effect on GABAergic System. Eur. J. Med. Chem. 2014, 86, 690-699. [CrossRef]

18. Abuo-Rahma, G.E.-D.A.A.; Abdel-Aziz, M.; Beshr, E.A.M.; Ali, T.F.S. 1,2,4-Triazole/Oxime Hybrids as New Strategy for Nitric Oxide Donors: Synthesis, Anti-Inflammatory, Ulceroginicity and Antiproliferative Activities. Eur. J. Med. Chem. 2014, 71, 185-198. [CrossRef]

19. Mohan Krishna, K.; Inturi, B.; Pujar, G.V.; Purohit, M.N.; Vijaykumar, G.S. Design, Synthesis and 3D-QSAR Studies of New Diphenylamine Containing 1,2,4-Triazoles as Potential Antitubercular Agents. Eur. J. Med. Chem. 2014, 84, 516-529. [CrossRef]

20. Láinez, M.J. Rizatriptan in the Treatment of Migraine. Neuropsychiatr. Dis. Treat. 2006, 2, 247-259. [CrossRef]

21. Hassan, G.S.; El-Messery, S.M.; Al-Omary, F.A.M.; Al-Rashood, S.T.; Shabayek, M.I.; Abulfadl, Y.S.; Habib, E.-S.E.; El-Hallouty, S.M.; Fayad, W.; Mohamed, K.M.; et al. Nonclassical Antifolates, Part 4. 5-(2-Aminothiazol-4-Yl)-4-Phenyl-4H-1,2,4-Triazole-3Thiols as a New Class of DHFR Inhibitors: Synthesis, Biological Evaluation and Molecular Modeling Study. Eur. J. Med. Chem. 2013, 66, 135-145. [CrossRef] [PubMed]

22. Küçükgüzel, İ.; Rollas, S.; Çevikbaş, A. Synthesis and Characterization of Certain Thiourea Derivatives Starting from 1,2,4Triazoline-3-Thiones as Potential Antibacterial and Antifungal Agents. Drug Metabol. Drug Interact. 1995, 12. [CrossRef] [PubMed]

23. Franklim, T.; Freire-de-Lima, L.; de Nazareth Sá Diniz, J.; Previato, J.; Castro, R.; Mendonça-Previato, L.; de Lima, M. Design, Synthesis and Trypanocidal Evaluation of Novel 1,2,4-Triazoles-3-Thiones Derived from Natural Piperine. Molecules 2013, 18, 6366-6382. [CrossRef] [PubMed] 
24. Kalluraya, B.; Isloor, A.M.; Shenoy, S. Synthesis and Biological Activity of 6-Substituted-3-[4-(3-Substituted Pyrazolidene) Hydrazino-4-Thiazolyl] Coumarins. Indian J. Heterocycl. Chem. 2001, 11, 159-162.

25. Isloor, A.M.; Kalluraya, B.; Shetty, P. Regioselective Reaction: Synthesis, Characterization and Pharmacological Studies of Some New Mannich Bases Derived from 1,2,4-Triazoles. Eur. J. Med. Chem. 2009, 44, 3784-3787. [CrossRef]

26. Enguehard, C.; Renou, J.-L.; Allouchi, H.; Leger, J.-M.; Gueiffier, A. Synthesis of Diaryl-Substituted Imidazo[1, 2-a]Pyridines Designed as Potential Aromatase Inhibitors. Chem. Pharm. Bull. 2000, 48, 935-940. [CrossRef] [PubMed]

27. Sánchez-Moreno, M.; Gómez-Contreras, F.; Navarro, P.; Marín, C.; Ramírez-Macías, I.; Olmo, F.; Sanz, A.M.; Campayo, L.; Cano, C.; Yunta, M.J.R. In Vitro Leishmanicidal Activity of Imidazole- or Pyrazole-Based Benzo[g]Phthalazine Derivatives against Leishmania Infantum and Leishmania Braziliensis Species. J. Antimicrob. Chemother. 2012, 67, 387-397. [CrossRef]

28. Khabnadideh, S.; Rezaei, Z.; Motazedian, M.H.; Eskandari, M. Synthesis of Metronidazole Derivatives as Antigiardiasis Agents. DARU J. Pharm. Sci. 2007, 15, 17-20.

29. Stover, C.K.; Warrener, P.; VanDevanter, D.R.; Sherman, D.R.; Arain, T.M.; Langhorne, M.H.; Anderson, S.W.; Towell, J.A.; Yuan, Y.; McMurray, D.N.; et al. A Small-Molecule Nitroimidazopyran Drug Candidate for the Treatment of Tuberculosis. Nature 2000, 405, 962-966. [CrossRef]

30. Łażewska, D.; Więcek, M.; Ligneau, X.; Kottke, T.; Weizel, L.; Seifert, R.; Schunack, W.; Stark, H.; Kieć-Kononowicz, K. Histamine H3 and H4 Receptor Affinity of Branched 3-(1H-Imidazol-4-Yl)Propyl N-Alkylcarbamates. Bioorg. Med. Chem. Lett. 2009, 19, 6682-6685. [CrossRef]

31. Galley, G.; Stalder, H.; Goergler, A.; Hoener, M.C.; Norcross, R.D. Optimisation of Imidazole Compounds as Selective TAAR1 Agonists: Discovery of RO5073012. Bioorg. Med. Chem. Lett. 2012, 22, 5244-5248. [CrossRef]

32. Hancock, A.A.; Bennani, Y.L.; Bush, E.N.; Esbenshade, T.A.; Faghih, R.; Fox, G.B.; Jacobson, P.; Knourek-Segel, V.; Krueger, K.M.; Nuss, M.E.; et al. Antiobesity Effects of A-331440, a Novel Non-Imidazole Histamine H3 Receptor Antagonist. Eur. J. Pharmacol. 2004, 487, 183-197. [CrossRef]

33. Hadizadeh, F.; Hassanabad, Z.F.; Bamshad, M.; Poorsoghat, H.; Hassanabad, M.F. Synthesis and Antihypertensive Activity of New 1,4-Dihydropyridines. Indian J. Chem. 2005, 44B, 2343-2347. [CrossRef]

34. Göker, H.; Kuş, C.; Boykin, D.W.; Yildiz, S.; Altanlar, N. Synthesis of Some New 2-Substituted-Phenyl-1H-Benzimidazole-5Carbonitriles and Their Potent Activity against Candida Species. Bioorg. Med. Chem. 2002, 10, 2589-2596. [CrossRef]

35. Insuasty, B.; Fernandez, F.; Quiroga, J.; Martinez, R.; Gavino, R.; Angeles, E. Reaction of 1,2-Diaminobenzimidazole with 1-Aryl-2-Bromo-3-Phenylpropanone. Synthesis of 2-Aryl-3-Benzyl-9-Aminoimidazo[1,2-a] Benzimidazoles. Heterocycl. Commun. 2002, 8. [CrossRef]

36. Zhou, J.; Song, Y.; Yang, Y.; Zhu, Y.; Tu, S. One-Step Synthesis of 2-Aryl-4,5-diphenylimidazoles Under Microwave Irradiation. Synth. Commun. 2005, 35, 1369-1373. [CrossRef]

37. Rastkari, N.; Sharifzadeh, M. Anticonvulsant Activities of New 1,4-Dihydropyridine Derivatives Containing 4-Nitroimidazolyl Substituents. Daru J. Pharm. Sci. 2004, 12, 81-86.

38. Mishra, R.; Ganguly, S. Imidazole as an Anti-Epileptic: An Overview. Med. Chem. Res. 2012, 21, 3929-3939. [CrossRef]

39. Sharma, D.; Narasimhan, B.; Kumar, P.; Judge, V.; Narang, R.; De Clercq, E.; Balzarini, J. Synthesis, Antimicrobial and Antiviral Evaluation of Substituted Imidazole Derivatives. Eur. J. Med. Chem. 2009, 44, 2347-2353. [CrossRef] [PubMed]

40. Zhang, P.; Zhang, N.; Korba, B.E.; Hosmane, R.S. Synthesis and in Vitro Anti-Hepatitis B and C Virus Activities of Ring-Expanded ('Fat') Nucleobase Analogues Containing the Imidazo[4,5-e][1,3]Diazepine-4,8-Dione Ring System. Bioorg. Med. Chem. Lett. 2005, 15, 5397-5401. [CrossRef] [PubMed]

41. Avram, S.; Svab, I.; Bologa, C.; Flonta, M.-L. Correlation between the Predicted and the Observed Biological Activity of the Symmetric and Nonsymmetric Cyclic Urea Derivatives Used as HIV-1 Protease Inhibitors. A 3D-QSAR-CoMFA Method for New Antiviral Drug Design. J. Cell. Mol. Med. 2003, 7, 287-296. [CrossRef]

42. Chang, L.L.; Sidler, K.L.; Cascieri, M.A.; de Laszlo, S.; Koch, G.; Li, B.; MacCoss, M.; Mantlo, N.; O'Keefe, S.; Pang, M.; et al. Substituted Imidazoles as Glucagon Receptor Antagonists. Bioorg. Med. Chem. Lett. 2001, 11, 2549-2553. [CrossRef]

43. Baroniya, S.; Anwer, Z.; Sharma, P.K.; Dudhe, R.; Kumar, N. Recent Advancement in Imidazole as Anti Cancer Agents: A Review. Der Pharm. Sin. 2010, 11, 172-182.

44. Zhou, C.; Hassner, A. Synthesis and Anticancer Activity of Novel Chiral D-Glucose Derived Bis-Imidazoles and Their Analogs. Carbohydr. Res. 2001, 333, 313-326. [CrossRef]

45. Kathiravan, M.K.; Salake, A.B.; Chothe, A.S.; Dudhe, P.B.; Watode, R.P.; Mukta, M.S.; Gadhwe, S. The Biology and Chemistry of Antifungal Agents: A Review. Bioorg. Med. Chem. 2012, 20, 5678-5698. [CrossRef] [PubMed]

46. Zhai, B.; Lin, X. Recent Progress on Antifungal Drug Development. Curr. Pharm. Biotechnol. 2011, 12, 1255-1262. [CrossRef] [PubMed]

47. Cai, J.-L.; Lu, Y.-H.; Gan, L.-L.; Zhang, Y.-Y.; Zhou, C.-H. Recent Advance in the Research of Piperazine-Containing Compounds as Antimicrobial Agents. Chin. J. Antibiot. 2009, 34, 454-462.

48. Peng, X.-M.; Cai, G.-X.; Zhou, C.-H. Recent Developments in Azole Compounds as Antibacterial and Antifungal Agents. Curr. Top. Med. Chem. 2013, 13, 1963-2010. [CrossRef] [PubMed]

49. Hammond, N.L.; Choi, S.; Carvalho, P.; Liu, H.; Khan, S.; Avery, M.A. Synthesis and Biological Evaluation of a Novel Anti-Malarial Lead. Med. Chem. Res. 2011, 20, 401-407. [CrossRef] 
50. Glans, L.; Ehnbom, A.; de Kock, C.; Martínez, A.; Estrada, J.; Smith, P.J.; Haukka, M.; Sánchez-Delgado, R.A.; Nordlander, E. Ruthenium(Ii) Arene Complexes with Chelating Chloroquine Analogue Ligands: Synthesis, Characterization and in Vitro Antimalarial Activity. Dalton Trans. 2012, 41, 2764. [CrossRef] [PubMed]

51. Dutta, S. Synthesis and Anthelmintic Activity of Some Novel 2-Substituted-4,5-Diphenyl Imidazoles. Acta Pharm. 2010, 60, 229-235. [CrossRef] [PubMed]

52. Baliani, A.; Bueno, G.J.; Stewart, M.L.; Yardley, V.; Brun, R.; Barrett, M.P.; Gilbert, I.H. Design and Synthesis of a Series of Melamine-Based Nitroheterocycles with Activity against Trypanosomatid Parasites. J. Med. Chem. 2005, 48, 5570-5579. [CrossRef] [PubMed]

53. Baliani, A.; Peal, V.; Gros, L.; Brun, R.; Kaiser, M.; Barrett, M.P.; Gilbert, I.H. Novel Functionalized Melamine-Based Nitroheterocycles: Synthesis and Activity against Trypanosomatid Parasites. Org. Biomol. Chem. 2009, 7, 1154. [CrossRef]

54. Sánchez-Moreno, M.; Gómez-Contreras, F.; Navarro, P.; Marín, C.; Olmo, F.; Yunta, M.J.R.; Sanz, A.M.; Rosales, M.J.; Cano, C.; Campayo, L. Phthalazine Derivatives Containing Imidazole Rings Behave as Fe-SOD Inhibitors and Show Remarkable Anti- T. Cruzi Activity in Immunodeficient-Mouse Mode of Infection. J. Med. Chem. 2012, 55, 9900-9913. [CrossRef] [PubMed]

55. Bhatt, H.G.; Agrawal, Y.K.; Raval, H.G.; Manna, K.; Desai, P.R. Histamine H4 Receptor: A Novel Therapeutic Target for Immune and Allergic Responses. Mini-Rev. Med. Chem. 2010, 10, 1293-1308. [CrossRef] [PubMed]

56. Tiligada, E.; Zampeli, E.; Sander, K.; Stark, H. Histamine H3 and H4 Receptors as Novel Drug Targets. Expert Opin. Investig. Drugs 2009, 18, 1519-1531. [CrossRef]

57. Geyer, R.; Buschauer, A. Synthesis and Histamine H3 and H4 Receptor Activity of Conformationally Restricted Cyanoguanidines Related to UR-PI376. Arch. Pharm. 2011, 344, 775-785. [CrossRef]

58. Hack, S.; Wörlein, B.; Höfner, G.; Pabel, J.; Wanner, K.T. Development of Imidazole Alkanoic Acids as MGAT3 Selective GABA Uptake Inhibitors. Eur. J. Med. Chem. 2011, 46, 1483-1498. [CrossRef]

59. Seo, H.J.; Park, E.-J.; Kim, M.J.; Kang, S.Y.; Lee, S.H.; Kim, H.J.; Lee, K.N.; Jung, M.E.; Lee, M.; Kim, M.-S.; et al. Design and Synthesis of Novel Arylpiperazine Derivatives Containing the Imidazole Core Targeting 5-HT2A Receptor and 5-HT Transporter. J. Med. Chem. 2011, 54, 6305-6318. [CrossRef]

60. Gonçalves, A.E.; Bürger, C.; Amoah, S.K.S.; Tolardo, R.; Biavatti, M.W.; de Souza, M.M. The Antidepressant-like Effect of Hedyosmum Brasiliense and Its Sesquiterpene Lactone, Podoandin in Mice: Evidence for the Involvement of Adrenergic, Dopaminergic and Serotonergic Systems. Eur. J. Pharmacol. 2012, 674, 307-314. [CrossRef]

61. Lakatos, A.; Gyurcsik, B.; Nagy, N.V.; Csendes, Z.; Wéber, E.; Fülöp, L.; Kiss, T. Histidine-Rich Branched Peptides as Cu(Ii) and Zn(Ii) Chelators with Potential Therapeutic Application in Alzheimer's Disease. Dalton Trans. 2012, 41, 1713-1726. [CrossRef]

62. Tyagarajan, S.; Chakravarty, P.K.; Zhou, B.; Fisher, M.H.; Wyvratt, M.J.; Lyons, K.; Klatt, T.; Li, X.; Kumar, S.; Williams, B.; et al. Substituted Biaryl Oxazoles, Imidazoles, and Thiazoles as Sodium Channel Blockers. Bioorg. Med. Chem. Lett. 2010, 20, 5536-5540. [CrossRef]

63. Hanna-Elias, A.; Manallack, D.T.; Berque-Bestel, I.; Irving, H.R.; Coupar, I.M.; Iskander, M.N. Synthesis and Preliminary Screening of Novel Tryptamines as 5-HT4 Receptor Ligands. Curr. Med. Chem. 2010, 17, 2775-2787. [CrossRef] [PubMed]

64. Galambos, J.; Wágner, G.; Nógrádi, K.; Bielik, A.; Molnár, L.; Bobok, A.; Horváth, A.; Kiss, B.; Kolok, S.; Nagy, J.; et al. Carbamoyloximes as Novel Non-Competitive MGlu5 Receptor Antagonists. Bioorg. Med. Chem. Lett. 2010, 20, 4371-4375. [CrossRef] [PubMed]

65. Prasad, J.; Pathak, M.B.; Panday, S.K. An Efficient and Straight Forward Synthesis of (5S)-1-Benzyl-5- (1H-Imidazol-1-Ylmethyl)-2Pyrrolidinone (MM1): A Novel Antihypertensive Agent. Med. Chem. Res. 2012, 21, 321-324. [CrossRef]

66. Agelis, G.; Resvani, A.; Durdagi, S.; Spyridaki, K.; Tůmová, T.; Slaninová, J.; Giannopoulos, P.; Vlahakos, D.; Liapakis, G.; Mavromoustakos, T.; et al. The Discovery of New Potent Non-Peptide Angiotensin II AT1 Receptor Blockers: A Concise Synthesis, Molecular Docking Studies and Biological Evaluation of N-Substituted 5-Butylimidazole Derivatives. Eur. J. Med. Chem. 2012, 55, 358-374. [CrossRef] [PubMed]

67. Bhandari, K.; Srinivas, N.; Shiva Keshava, G.B.; Shukla, P.K. Tetrahydronaphthyl Azole Oxime Ethers: The Conformationally Rigid Analogues of Oxiconazole as Antibacterials. Eur. J. Med. Chem. 2009, 44, 437-447. [CrossRef]

68. Le, T.M.; Huynh, T.; Endre, G.; Szekeres, A.; Fülöp, F.; Szakonyi, Z. Stereoselective Synthesis and Application of Isopulegol-Based Bi- and Trifunctional Chiral Compounds. RSC Adv. 2020, 10, 38468-38477. [CrossRef]

69. Nazimova, E.; Pavlova, A.; Mikhalchenko, O.; Il'ina, I.; Korchagina, D.; Tolstikova, T.; Volcho, K.; Salakhutdinov, N. Discovery of Highly Potent Analgesic Activity of Isopulegol-Derived (2R,4aR,7R,8aR)-4,7-Dimethyl-2-(Thiophen-2-Yl)Octahydro-2H-Chromen4-Ol. Med. Chem. Res. 2016, 25, 1369-1383. [CrossRef]

70. Moreira, J.A.; Corrêa, A.G. Enantioselective Synthesis of Three Stereoisomers of 5,9-Dimethylpentadecane, Sex Pheromone Component of Leucoptera Coffeella, from (-)-Isopulegol. Tetrahedron Asymmetry 2003, 14, 3787-3795. [CrossRef]

71. Rigamonti, M.G.; Gatti, F.G. Stereoselective Synthesis of Hernandulcin, Peroxylippidulcine A, Lippidulcines A, B and C and Taste Evaluation. Beilstein J. Org. Chem. 2015, 11, 2117-2124. [CrossRef] [PubMed]

72. Friedrich, D.; Bohlmann, F. Total Synthesis of Various Elemanolides. Tetrahedron 1988, 44, 1369-1392. [CrossRef]

73. Travis, B.R.; Narayan, R.S.; Borhan, B. Osmium Tetroxide-Promoted Catalytic Oxidative Cleavage of Olefins: An Organometallic Ozonolysis. J. Am. Chem. Soc. 2002, 124, 3824-3825. [CrossRef] [PubMed] 
74. Costa, G.N.; Carrilho, R.M.B.; Dias, L.D.; Viana, J.C.; Aquino, G.L.B.; Pineiro, M.; Pereira, M.M. Highly Efficient Rh(I)/TrisBinaphthyl Monophosphite Catalysts for Hydroformylation of Sterically Hindered Alkyl Olefins. J. Mol. Catal. Chem. 2016, 416, 73-80. [CrossRef]

75. Ren, B.; Wang, M.; Liu, J.; Ge, J.; Dong, H. Enhanced Basicity of Ag2O by Coordination to Soft Anions. ChemCatChem 2015, 7, 761-765. [CrossRef]

76. Hussain, H.; Al-Harrasi, A.; Green, I.R.; Ahmed, I.; Abbas, G.; Rehman, N.U. Meta-Chloroperbenzoic Acid (MCPBA): A Versatile Reagent in Organic Synthesis. RSC Adv. 2014, 4, 12882-12917. [CrossRef]

77. Katz, S.J.; Bergmeier, S.C. Convenient Methods for the Hydrolysis of Oxazolidinones to Vicinal Aminoalcohols. Tetrahedron Lett. 2002, 43, 557-559. [CrossRef]

78. Shivani, P.B.; Pujala, B.; Chakraborti, A.K. Zinc(II) Perchlorate Hexahydrate Catalyzed Opening of Epoxide Ring by Amines: Applications to Synthesis of (RS)/(R)-Propranolols and (RS )/(R)/(S)-Naftopidils. J. Org. Chem. 2007, 72, 3713-3722. [CrossRef]

79. Azizi, N.; Mehrazma, S.; Saidi, M.R. A Simple, Highly Regioselective and Efficient Reaction of Indole with Epoxides under Solvent-Free Conditions. Can. J. Chem. 2006, 84, 800-803. [CrossRef]

80. Azizi, N.; Mirmashhori, B.; Saidi, M.R. Lithium Perchlorate Promoted Highly Regioselective Ring Opening of Epoxides under Solvent-Free Conditions. Catal. Commun. 2007, 8, 2198-2203. [CrossRef]

81. Upadhayaya, R.S.; Lahore, S.V.; Sayyed, A.Y.; Dixit, S.S.; Shinde, P.D.; Chattopadhyaya, J. Conformationally-Constrained Indeno[2,1-c]Quinolines-A New Class of Anti-Mycobacterial Agents. Org. Biomol. Chem. 2010, 8, 2180. [CrossRef] [PubMed]

82. Wang, S.; Xie, Z.; Li, M.; Wang, C. $\mathrm{K}_{2} \mathrm{CO}_{3}$-promoted Ring-opening/Cyclization Reactions of Multi-substituted Donor-acceptor Cyclopropanes with Thiourea: Access to 2-amino-4,6-diarylnicotinonitrile Derivatives. ChemistrySelect 2020, 5, 6011-6015. [CrossRef]

83. Gonda, T.; Szakonyi, Z.; Csámpai, A.; Haukka, M.; Fülöp, F. Stereoselective Synthesis and Application of Tridentate Aminodiols Derived from (+)-Pulegone. Tetrahedron Asymmetry 2016, 27, 480-486. [CrossRef]

84. Szakonyi, Z.; Csőr, Á.; Csámpai, A.; Fülöp, F. Stereoselective Synthesis and Modelling-Driven Optimisation of Carane-Based Aminodiols and 1,3-Oxazines as Catalysts for the Enantioselective Addition of Diethylzinc to Benzaldehyde. Chem. Eur. J. 2016, 22, 7163-7173. [CrossRef] [PubMed]

85. Macías, F.A.; Velasco, R.F.; Álvarez, J.A.; Castellano, D.; Galindo, J.C.G. Synthesis of Melampolides and Cis,Cis-Germacranolides as Natural Herbicide Models. Tetrahedron 2004, 60, 8477-8488. [CrossRef]

86. Chen, K.; Baran, P.S. Total Synthesis of Eudesmane Terpenes by Site-Selective C-H Oxidations. Nature 2009, 459, 824-828. [CrossRef] [PubMed]

87. Sajiki, H.; Hattori, K.; Hirota, K. Highly Chemoselective Hydrogenation with Retention of the Epoxide Function Using a Heterogeneous Pd/C-Ethylenediamine Catalyst and THF. Chem. Eur. J. 2000, 6, 2200-2204. [CrossRef]

88. Ley, S.V.; Stewart-Liddon, A.J.P.; Pears, D.; Perni, R.H.; Treacher, K. Hydrogenation of Aromatic Ketones, Aldehydes, and Epoxides with Hydrogen and Pd(0)EnCat ${ }^{\mathrm{TM}} 30 \mathrm{NP}$. Beilstein J. Org. Chem. 2006, 2. [CrossRef]

89. Rani, N.; Sharma, A.; Singh, R. Imidazoles as Promising Scaffolds for Antibacterial Activity: A Review. Mini-Rev. Med. Chem. 2013, 13, 1812-1835. [CrossRef]

90. Borgers, M. Mechanism of Action of Antifungal Drugs, with Special Reference to the Imidazole Derivatives. Clin. Infect. Dis. 1980, 2, 520-534. [CrossRef]

91. Mamolo, M.G.; Zampieri, D.; Falagiani, V.; Vio, L.; Fermeglia, M.; Ferrone, M.; Pricl, S.; Banfi, E.; Scialino, G. Antifungal and Antimycobacterial Activity of New N1-[1-Aryl-2-(1H-Imidazol-1-Yl and 1H-1,2,4-Triazol-1-Yl)-Ethylidene]-Pyridine-2Carboxamidrazone Derivatives: A Combined Experimental and Computational Approach. Arkivoc 2004, 2004, 231-250. [CrossRef]

92. Weinstein, M.P. Methods for Dilution Antimicrobial Susceptibility Tests for Bacteria That Grow Aerobically. Available online: https: / / clsi.org/standards/products/microbiology/documents/m07/ (accessed on 2 February 2021).

93. Béni, Z.; Dékány, M.; Kovács, B.; Csupor-Löffler, B.; Zomborszki, Z.; Kerekes, E.; Szekeres, A.; Urbán, E.; Hohmann, J.; Ványolós, A. Bioactivity-Guided Isolation of Antimicrobial and Antioxidant Metabolites from the Mushroom Tapinella Atrotomentosa. Molecules 2018, 23, 1082. [CrossRef] [PubMed] 